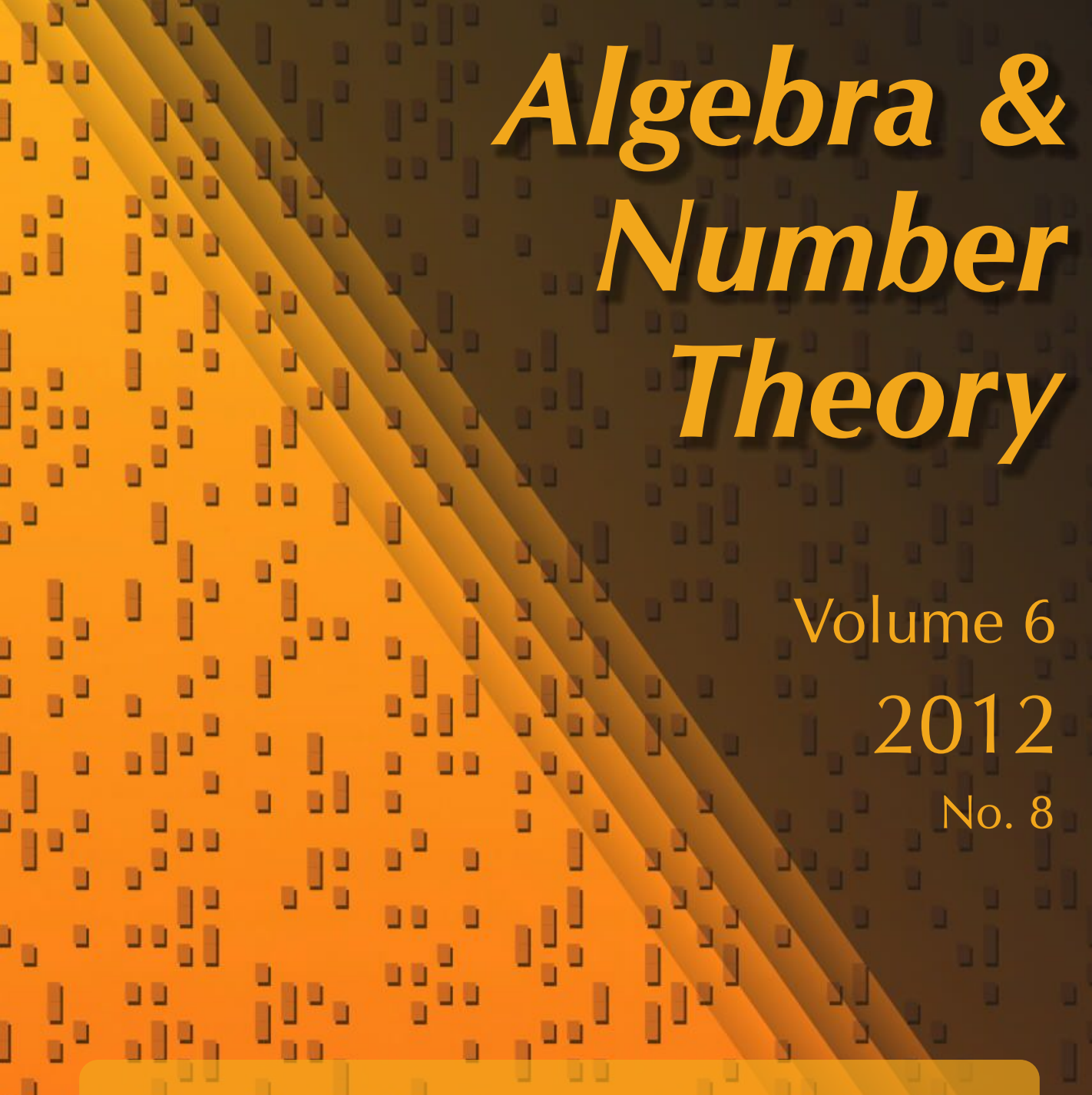

Abelian varieties and Weil representations

Sug Woo Shin

」

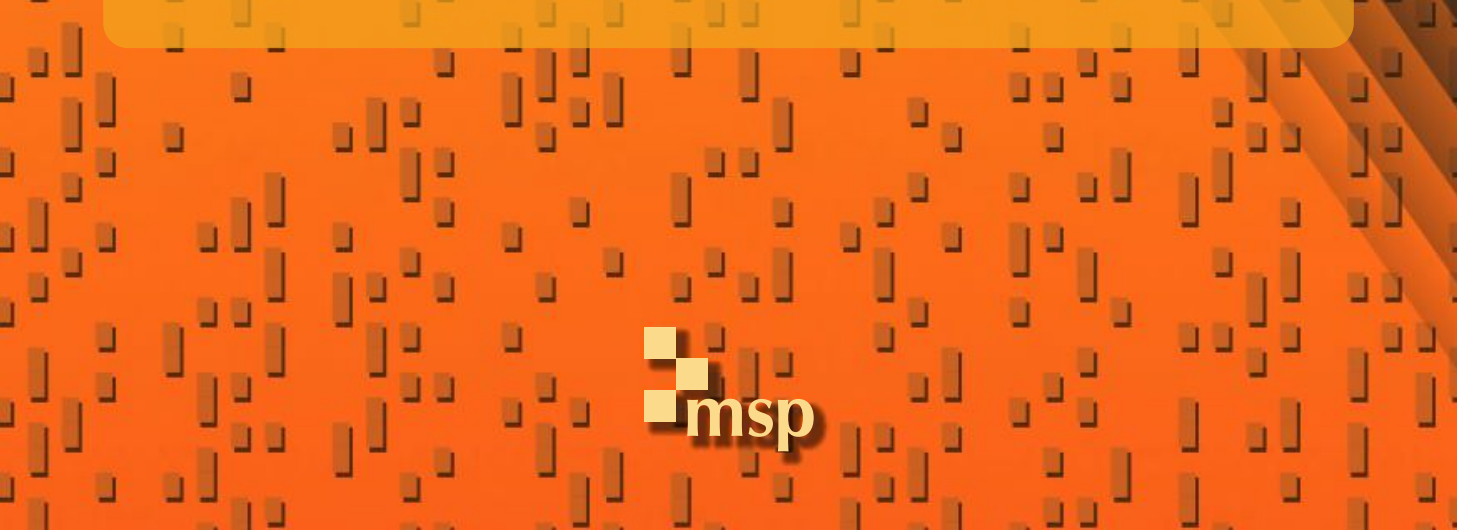




\title{
Abelian varieties and Weil representations
}

\author{
Sug Woo Shin
}

\begin{abstract}
The main goal of this article is to construct and study a family of Weil representations over an arbitrary locally noetherian scheme without restriction on characteristic. The key point is to recast the classical theory in the schemetheoretic setting. As in work of Mumford, Moret-Bailly and others, a Heisenberg group (scheme) and its representation can be naturally constructed from a pair of an abelian scheme and a nondegenerate line bundle, replacing the role of a symplectic vector space. Once enough is understood about the Heisenberg group and its representations (e.g., the analogue of the Stone-von Neumann theorem), it is not difficult to produce the Weil representation of a metaplectic group (functor) from them. As an interesting consequence (when the base scheme is Spec $\overline{\mathbb{F}}_{p}$ ), we obtain the new notion of mod $p$ Weil representations of $p$-adic metaplectic groups on $\overline{\mathbb{F}}_{p}$-vector spaces. The mod $p$ Weil representations admit an alternative construction starting from a $p$-divisible group with a symplectic pairing.

We have been motivated by a few possible applications, including a conjectural $\bmod p$ theta correspondence for $p$-adic reductive groups and a geometric approach to the (classical) theta correspondence.
\end{abstract}

\section{Introduction}

For a quick overview of contents and results, see Section $1 \mathrm{H}$.

1A. Motivation from theta correspondence. The Heisenberg groups, their representations and the Weil representations (also called oscillator or metaplectic representations) play interesting roles in a wide range of mathematics. In the context of number theory and representation theory, they give rise to the theta correspondence, which enables us to relate automorphic forms or representations of one connected reductive group (or its covering group) to those of another group. It not only helps to establish instances of the Langlands functoriality but also reveals

The author's work was supported by the National Science Foundation during his stay at the Institute for Advanced Study under agreement No. DMS-0635607. Any opinions, findings and conclusions or recommendations expressed in this material are those of the author and do not necessarily reflect the views of the National Science Foundation.

MSC2010: primary 11F27; secondary 11G10, 14K25.

Keywords: abelian varieties, Weil representations, Heisenberg groups. 
deep information about arithmetic invariants and as such has led to numerous profound applications. The theta correspondence has been developed very well in both local and global contexts (namely, for $p$-adic/real groups and adelic groups, respectively), though there are still many open questions, for representations on vector spaces over $\mathbb{C}$ (or an algebraically closed field of characteristic 0 ).

On the other hand, there has been growing interest in the representations of $p$-adic reductive groups on vector spaces over $\overline{\mathbb{F}}_{l}(l \neq p)$ and $\overline{\mathbb{F}}_{p}$ (as well as representations with $p$-adic analytic structure) in connection with Galois theory as part of the extended Langlands philosophy under the motto "mod $l, \bmod p$ and $p$-adic local Langlands program". From the global perspective, one seeks the theta correspondence for mod $p$ or $p$-adic automorphic forms. ${ }^{1}$ Thus, it is a very natural question to ask whether there is a reasonable theory of local and global theta correspondence for representations on $\overline{\mathbb{F}}_{l}$ and $\overline{\mathbb{F}}_{p}$ vector spaces and more ambitiously for representations of $p$-adic analytic nature.

In the classical theory, the following basic ingredients are needed to formulate the local theta correspondence for $p$-adic groups. The global setup is similar. (Unfortunately the exceptional theta correspondence is not going to be considered in our work.) We need

(i) a $p$-adic Heisenberg group arising from a symplectic vector space $(V,\langle\cdot, \cdot\rangle)$ over $\mathbb{Q}_{p}$,

(ii) the Stone-von Neumann theorem and Schur's lemma for representations of the Heisenberg group,

(iii) the Weil representation of the $p$-adic metaplectic group $\operatorname{Mp}(V,\langle\cdot, \cdot\rangle)$, and

(iv) reductive dual pairs in $\operatorname{Sp}(V,\langle\cdot, \cdot\rangle)$.

It is natural to try to extend (i)-(iv) to a more general setting. The current paper will do this for (i)-(iii), leaving (iv) (and a conjectural mod $p$ theta correspondence) to a sequel [Shin $\geq 2012]$.

1B. Mod p Weil representations, prelude. Let us briefly point out some difficulty when trying to construct the Weil representation of a $p$-adic metaplectic group on an $\overline{\mathbb{F}}_{p}$-vector space, which was not done before but is a special case of our results. There would be two naïve approaches. If one tries to define an $\overline{\mathbb{F}}_{p}$-version of a classical $p$-adic Heisenberg group (e.g., [Mœglin et al. 1987, Chapter 2]) by replacing the role of $\mathbb{C}$ by $\overline{\mathbb{F}}_{p}$, it is impossible to obtain a reasonable group, ruling out (ii) above. For instance, every continuous additive character $\mathbb{Q}_{p} \rightarrow \overline{\mathbb{F}}_{p}^{\times}$is trivial.

\footnotetext{
${ }^{1}$ The $p$-adic version of the Shimura-Shintani correspondence was studied in [Stevens 1994; Ramsey 2009; Park 2010]. Their work interpolates the classical correspondence $p$-adically and does not require much use of representation theory. The author does not know yet whether or how their work could be interpreted in the framework of representation theory.
} 
Another approach would be to take an explicit (Schrödinger or lattice) model for the Weil representation and switch the coefficient from $\mathbb{C}$ to $\overline{\mathbb{F}}_{p}$. Then the problem is that the group actions are no longer well defined. In the Schrödinger model, some group action is given by Fourier transform, which cannot be defined for $\overline{\mathbb{F}}_{p}$-valued functions on a $p$-adic group. (See Remark 7.9 for a related discussion.) In the lattice model, the formula involves $p$ in the denominator, which no longer makes sense. It is not immediately clear how to fix these problems unless new ideas are introduced.

1C. Geometric construction via Mumford's theory. We remedy the situation by giving a uniform geometric construction of (i)-(iii) regardless of the characteristic of the coefficient field, starting from an abelian scheme $A \rightarrow S$ and a nondegenerate line bundle $L$ instead of a symplectic vector space. In the local case, the effect is roughly to replace $(V,\langle\cdot, \cdot\rangle)$ by the rational $p$-adic Tate module $V_{p} A$ of $A$ with $L$-Weil pairing. (Here $V_{p} A$ is regarded as an ind-group scheme as explained in Section 3A.) The construction makes sense even in characteristic $p$; it just behaves differently. (For an analogy, think about $A\left[p^{\infty}\right]$ in characteristic $p$ and away from $p$.) Actually (i) and (ii) are basically treated in Mumford's theory of abelian varieties and theta functions. (As the results are often not in the desired generality in the literature, we fill the gaps along the way. See the next paragraph.) Once (i) and (ii) are done, (iii) is obtained without much difficulty. The theory is so flexible as to allow the construction of the objects (i)-(iii) over an arbitrary locally noetherian base scheme $S$.

Sections 2-4 of our paper follow the approach of [Mumford 2007, §§3-5] and [Moret-Bailly 1985, §5] closely while adapting several facts in the classical theory of theta correspondence (e.g., [Mœglin et al. 1987]) to the geometric setting. In [Mumford 2007, §§3-5], the Heisenberg groups and their representations are studied mostly over an (algebraically closed) field, and the scheme-theoretic approach in the relative setting is only sketched on a few pages. Moret-Bailly consistently works in the relative setting, but the theory is treated only at finite level. Our contribution is to carry out the construction and justify necessary facts (e.g., Theorems 1.1 and 1.2) at infinite level (in a $p$-adic or a finite adelic limit). As a byproduct we obtain the (dual) lattice model over a general locally noetherian base scheme and deduce the restriction property of Heisenberg and Weil representations (Section 4E and Lemma 5.10) from the Künneth formula. (It turns out that lattice models always exist, but Schrödinger models are often missing.) We can also make sense of matrix coefficients and dual representations in this generality. It is hoped that the geometric interpretation will shed light on some facts well known by other methods.

Our work is definitely not the first attempt toward a geometric construction of Weil representations. This was considered in an unpublished manuscript of Harris [1987]. (It appears that the manuscript was planned to include an application to some cases 
of the symplectic-orthogonal theta correspondence, but that part was not written to our knowledge.) His approach to Heisenberg groups and representations closely follows that of Mumford [1966, 1967a, 1967b] and works only in characteristic 0 (even though ideas are often generalizable). Hence, his setting is simpler than ours, and many scheme-theoretic issues do not arise there. His innovation is to construct a Weil representation in the way that it is closely tied with the geometry of Siegel modular varieties. On the other hand, our construction is so general that it applies to almost any families of abelian varieties, but when specialized to the universal abelian scheme over a Siegel modular variety, the two constructions of Weil representations by us and by Harris are orthogonal in some sense.

From a different perspective and motivation, [Gurevich and Hadani 2007] constructs classical Weil representations for finite metaplectic groups as perverse sheaves (Deligne's idea), and the function field analogue is dealt with in [Lysenko 2006; Lafforgue and Lysenko 2009], for instance. Their constructions are quite different from ours and do not seem to carry over to the number field case. In the converse direction, our construction does not work in the function field case either. The basic reason is that the $p$-adic symplectic (or metaplectic) group in our setting appears as the automorphism group of a rational $p$-adic Tate module, which is a vector space over $\mathbb{Q}_{p}$ rather than something like $\mathbb{F}_{p}((t))$.

In our setup, symplectic groups and metaplectic groups are defined as group functors varying over the base. By introducing a level structure, we can trivialize the rational Tate module (ind-scheme), which has the effect that those group functors may be identified with constant families of groups. When the base is $\operatorname{Spec} \mathbb{C}$, we precisely recover the classical notion of (i)-(iv) from our construction.

It is worth emphasizing that we have completely avoided the use of harmonic analysis. This is only natural for our method to work in all characteristics uniformly. In this regard, even when specialized to the classical case (over Spec $\mathbb{C}$ ), our construction of the Weil representation is different from the classical treatment (e.g., [Mœglin et al. 1987]).

As the reader can see, one of our crucial observations was to realize that Mumford's theory had the key to the main question raised in Section 1A. This may appear to be a simple idea, but when we consulted a few experts on theta correspondence, we learned that the idea was largely unnoticed though a similar idea must have been conceived by some experts (e.g., [Harris 1987]).

1D. Mod $p$ Weil representations. To study finite adelic objects, one may concentrate on one place at a time. So let us restrict ourselves to $p$-adic Heisenberg groups and $p$-adic metaplectic groups. By the Stone-von Neumann theorem (more precisely, its analogue in our setting), a family of Heisenberg representations, as well as that of Weil representations, tends to be a constant family (modulo the 
line bundle pulled back from the base). However, things do change when moving between points of different residue characteristic. Unsurprisingly, new phenomena essentially occur in characteristic $p$. (This is related to the fact that $A\left[p^{\infty}\right]$ is étale away from characteristic $p$.) It is worth noting that the Heisenberg and metaplectic groups vary significantly in characteristic $p$ as the isogeny type of $A\left[p^{\infty}\right]$ varies over fibers. On the other hand, over a base ring like $\overline{\mathbb{Z}}_{p}$, a classical Weil representation (over the generic fiber) specializes to a mod $p$ Weil representation (Section 7D).

In view of these new phenomena, we feel that it is fundamental to understand $\bmod p$ Weil representations, namely when the base is $\operatorname{Spec} \overline{\mathbb{F}}_{p}$. In order to make their local nature more transparent, we present an alternative construction of mod $p$ Weil representations using $p$-divisible groups instead of abelian schemes (Section 6D). Then lattice and Schrödinger models are studied in Sections 7B and 7C. There remains the question of whether the Schrödinger model exists in the nonordinary case (see the paragraph below Proposition 7.10). Another interesting question about the $p$-adic metaplectic group itself is whether it arises from a double covering of the $p$-adic symplectic group (see the questions in Section 5D).

1E. Weil representations of real metaplectic groups. Real Heisenberg groups and real metaplectic groups do not appear in this paper. This is not defective but quite natural if we want a uniform theory that works in positive characteristics as real groups are not expected to have nice representations on $\overline{\mathbb{F}}_{p}$-vector spaces. In the special case where the base is Spec $\mathbb{C}$, it is possible to extend the Heisenberg representations to real places (thereby one can define the real Weil representation) as explained in [Mumford 2007, §5, Application I] (also see Proposition 3.2 of the book).

1F. Summary of main results. Let $A$ be an abelian scheme over a locally noetherian scheme $S$. Let $f: L \rightarrow A$ be a symmetric nondegenerate line bundle of index $i$ over $A\left(0 \leq i \leq \operatorname{dim}_{S} A\right)$. Following Mumford, we construct the adelic Heisenberg group $\widehat{\mathscr{G}}(L)=\widehat{\mathscr{G}}(A, L)$ fitting in a short exact sequence $1 \rightarrow \mathbb{G}_{m} \rightarrow \widehat{\mathscr{G}}(L) \rightarrow V A \rightarrow 1$. A weight-1 representation of $\widehat{\mathscr{G}}(L)$ is defined to be a quasicoherent $O_{S}$-module equipped with $\widehat{\mathscr{G}}(L)$-action such that $\lambda \in \mathbb{G}_{m}$ acts by $\lambda$. An (adelic) Heisenberg representation of $\widehat{\mathscr{G}}(L)$ is an irreducible admissible and smooth $\widehat{\mathscr{G}}(L)$-representation of weight 1 that does not vanish anywhere on $S$. (Admissibility and smoothness are defined in Definitions 4.7 and 4.8.)

Theorem 1.1 (Stone-von Neumann theorem and Schur's lemma, Theorem 4.15). For any Heisenberg representation $\mathscr{H}$ of $\widehat{G}(L)$, there is an equivalence of categories

$$
\left(\begin{array}{c}
\text { weight } 1 \text { smooth } \\
\widehat{G}(L) \text {-representations }
\end{array}\right) \stackrel{\sim}{\rightarrow}\left(\begin{array}{c}
\text { quasicoherent } \\
\text { OS }_{S} \text {-modules }
\end{array}\right)
$$


given by $\mathcal{M} \mapsto \underline{\operatorname{Hom}}_{\widehat{G}(L)}(\mathcal{H}, \mathcal{M})$ and $\mathcal{N} \mapsto \mathscr{H} \otimes \mathcal{N}$, which are quasi-inverses of each other.

Theorem 1.2 (Construction of Heisenberg representations, Corollary 4.14). The $\mathrm{O}_{S}$-module $\widehat{\mathscr{V}}(L):=\lim _{\longrightarrow} R^{i} f_{*}\left(n^{*} L\right)$ is a Heisenberg representation of $\widehat{\mathscr{G}}(L)$.

Theorem 1.3 (Construction of Weil representations, Section 5A). For any Heisenberg representation Hef $\widehat{\mathscr{G}}(L)$, we can construct a "metaplectic" group functor $\mathrm{Mp}\left(V A, \hat{e}^{L}\right)$ sitting in a sequence of group functors on $(\mathrm{Sch} / S)$

$$
1 \rightarrow \mathbb{G}_{m} \rightarrow \underline{\mathrm{Mp}}\left(V A, \hat{e}^{L}\right) \rightarrow \underline{\mathrm{Sp}}\left(V A, \hat{e}^{L}\right) \rightarrow 1,
$$

which is an exact sequence of groups upon evaluation at any locally noetherian S-scheme.

Theorem 1.4 (Comparison with classical theory, Section 6A). In case $S=\operatorname{Spec} \mathbb{C}$, a choice of level structure for VA equipped with $L$-Weil pairing allows one to identify $\widehat{\mathscr{G}}(L), \widehat{\mathscr{V}}(L), \operatorname{Sp}\left(V A, \hat{e}^{L}\right)$ and $\mathrm{Mp}\left(V A, \hat{e}^{L}\right)$ with the following objects in the classical finite adelic setting: the Heisenberg group, Heisenberg representation, symplectic group and metaplectic group, respectively. (Here the metaplectic group is a central extension of the symplectic group by $\mathbb{C}^{\times}$as can be seen from (1-1).)

The preceding theorems are also valid in the $p$-adic setting instead of the finite adelic setting. (In particular, take limits over powers of $p$ rather than all positive integers, and use $V_{p} A$ in place of $V A$.) Moreover, the analogous construction works for $(\Sigma,\langle\cdot, \cdot\rangle)$ in place of $(A, L)$, where $\Sigma$ is a $p$-divisible group over $S$ with a symplectic pairing $\langle\cdot, \cdot\rangle$, granted that a Heisenberg representation exists for the Heisenberg group associated with $(\Sigma,\langle\cdot, \cdot\rangle)$. This is most interesting when $S$ is an $\mathbb{F}_{p}$-scheme. A Heisenberg representation for $(\Sigma,\langle\cdot, \cdot\rangle)$ can be exhibited when $S=$ Spec $k$ for an algebraically closed field $k$ of characteristic $p$ (but the author does not know in what generality it exists) and leads to a construction of a mod $p$ Weil representation of a $p$-adic group functor over $\operatorname{Spec} k$.

1G. Scope of applications and further developments. As we construct a family of Weil representations from a family of abelian varieties and line bundles, it would be natural to apply our results to the universal family of abelian varieties over moduli spaces such as Shimura varieties. This should be related to metaplectic automorphic forms and a worthy object already in characteristic 0 . We hope that our results will be of some use when studying theta correspondence via Shimura varieties by methods in algebraic geometry, for instance in the context of Kudla's program [2002].

When there is a Weil representation, it is very natural to consider a reductive dual pair and the resulting theta correspondence (Section 1A). In the sequel [Shin 
$\geq 2012$ ], we do this for the newly constructed $\bmod p$ Weil representation of a $p$-adic metaplectic group.

In order to access many cases of mod $p$ Weil representations and theta correspondence, a necessary step would be to explicate the models in Section 7 further, especially in the case of supersingular abelian varieties (or $p$-divisible groups).

1H. Contents and organization of the paper. This article is naturally divided into two parts. Under each part we have listed some of the main contents. The sequel [Shin $\geq 2012$ ] may be regarded as Part III.

Part I. Heisenberg groups and Heisenberg representations

- Construction of the $p$-adic or adelic Heisenberg group and Heisenberg representation from an abelian scheme $A$ and a nondegenerate line $L$ bundle over a locally noetherian scheme $S$. (Sections 2-4)

- A description of the Heisenberg group as $\mathbb{G}_{m} \times V A$ with a twisted group law, where $V A$ is the "rational Tate module", when $L$ is symmetric. (Section 3E)

- A study of the category of representations of the Heisenberg group, subsuming the Stone-von Neumann theorem and Schur's lemma. (Proposition 2.12 and Theorem 4.15)

Part II. Weil representations, level structures and explicit models

- Construction of the $p$-adic or adelic metaplectic group and the Weil representation over $S$. (Sections 5A and 5D)

- Comparison with classical theory via level structure. (Sections 6A-6B)

- Weil representations over $\overline{\mathbb{F}}_{p}$ of $p$-adic metaplectic group; Igusa level structure; an approach via a $p$-divisible group replacing the role of an abelian variety. (Sections 6C-6D)

- Study of lattice and Schrödinger models; examples. (Section 7)

1I. Notation and convention. If $S$ is a scheme, denote by $(\mathrm{Sch} / S),(\mathrm{Flat} / S)$ and (LocNoeth $/ S$ ) the categories of $S$-schemes, flat $S$-schemes and locally noetherian $S$-schemes, respectively. All fppf sheaves on $S$ in sets or groups are considered on a small fppf site. Their category is a full subcategory of the category functors from $\left(\right.$ Flat $/ S$ ) to the category of sets or groups. An $\mathrm{O}_{S}$-module always means a quasicoherent $\mathrm{O}_{S}$-module in this article and is often viewed as an fppf sheaf on $S$ as well. The category of $\mathrm{O}_{S}$-modules is denoted $\mathrm{QCoh}_{S}$.

An object of (Flat/S) may be viewed as an fppf sheaf in sets on $S$, and this induces a fully faithful functor. The underlined notation such as $\underline{\text { Hom, End and }}$ Aut denotes a sheaf or a functor (rather than just a set, a group, a ring, etc.) in the 
appropriate category determined by the context. Often $\mathrm{Mp}$ and $\mathrm{Sp}$ are defined as group functors on $(\mathrm{Sch} / S)$.

In this article we will usually work in $(\mathrm{Sch} / S)$ for a base scheme $S$. In particular, any morphism of schemes is always assumed to be an $S$-morphism, and a fiber product is taken over $S$ unless specified otherwise. The tensor product of two $\mathrm{O}_{S}$-modules is denoted by $\otimes\left(\right.$ rather than $\otimes_{\mathscr{O}_{S}}$ ) if there is no danger of confusion.

\section{Finite Heisenberg groups and their representations}

We use the following notation:

- $S$ is a scheme,

- $f: A \rightarrow S$ is an abelian scheme over $S$ of relative dimension $g \geq 1$,

- $f^{\vee}: A^{\vee} \rightarrow S$ is the dual abelian scheme (cf. [Faltings and Chai 1990, I.1]),

- $L$ is a line bundle over $A$,

- $T_{x}: A \times{ }_{S} T \rightarrow A \times{ }_{S} T$ is the translation by $x$, where $T$ is an $S$-scheme and $x \in A(T)$,

- $\lambda_{L}: A \rightarrow A^{\vee}$ is the morphism sending $x$ to $T_{x}^{*} L \otimes L^{-1}$.

When we think of $L$, we will often go between two equivalent viewpoints: either as an invertible sheaf $\mathscr{L}$ of $O_{S}$-modules on $A$ or as a line bundle equipped with a projection $\pi: L \rightarrow A$ (cf. [Mumford et al. 1994, I.3]). Given $L$, the corresponding $\mathscr{L}$ is described as $\mathscr{L}(U)=\left\{s: U \rightarrow L \mid \pi \circ s=\mathrm{id}_{U}\right\}$ for each open subscheme $U$ of $A$. By setting $L=\operatorname{Spec}\left(\oplus_{n \leq 0} \mathscr{L}^{\otimes n}\right.$ ) (relative spectrum over $A$ ), we recover $L$ from $\mathscr{L}$. In order to avoid cumbersome switch of notation, we just write $L$ for either $L$ or the corresponding $\mathscr{L}$.

\section{A. Nondegenerate line bundles.}

Definition 2.1. A line bundle $L$ over $A$ is (relatively) nondegenerate if $\lambda_{L}: A \rightarrow A^{\vee}$ is a finite morphism.

\section{Lemma 2.2. If $L$ is nondegenerate, then}

(i) $\lambda_{L}$ is an isogeny (a surjective quasifinite homomorphism of group schemes) and

(ii) $\operatorname{ker} \lambda_{L}$ is a finite flat group scheme over $S$.

Proof. We know that $\lambda_{L}$ is compatible with the group scheme structures. Surjectivity and quasifiniteness follow from the case of $S=\operatorname{Spec} k$ for a field $k$ when the result is well known (cf. [Bosch et al. 1990, Lemma 1, page 178]). Part (ii) is a consequence of the fact that any isogeny of abelian schemes is finite flat.

Lemma 2.3. The following are equivalent:

(i) L is nondegenerate in the above sense. 
(ii) For every point $s \in S$, the fiber $L_{s}$ over $A_{s}$ is nondegenerate (i.e., $\lambda_{L_{s}}$ is finite).

(iii) For every geometric point $\bar{s} \in S$, the fiber $L_{\bar{S}}$ over $A_{\bar{s}}$ is nondegenerate (i.e., $\lambda_{L_{\bar{S}}}$ is finite).

Proof. It is obvious that (i) implies (ii). By the flat base change theorem (applied to the base extension from $s$ to $\bar{s}$ ), (ii) and (iii) are equivalent. It remains to deduce (i) from (ii). Observe that (ii) implies that $\lambda_{L}$ is quasifinite. An easy application of the valuative criterion shows that $\lambda_{L}$ is proper. Hence, $\lambda_{L}$ is finite.

Lemma 2.4. Suppose that $A$ is defined over $S=\operatorname{Spec} k$, where $k$ is a field. For a nondegenerate line bundle $L$, there exists a unique integer $0 \leq \operatorname{ind}(L) \leq g$ (the index of $L)$ such that $H^{\operatorname{ind}(L)}(A, L) \neq 0$.

Proof. See [Mumford 1974, §16] when $k$ is algebraically closed. The general case is reduced to the algebraically closed case by the flat base change theorem.

In general the following result is well known. We present a proof as we were incompetent in finding a handy reference.

Lemma 2.5. Suppose that $S$ is locally noetherian. Let $L$ be a nondegenerate line bundle over $A$. The index function $s \mapsto \operatorname{ind}\left(L_{s}\right)$ from $S$ to $\mathbb{Z}$ is locally constant (with Zariski topology on $S$ ).

Proof. As the question is local, we may assume that $S$ is noetherian and connected. We know that $s \mapsto \operatorname{dim} H^{i}\left(A_{s}, L_{s}\right)$ is upper semicontinuous and that $s \mapsto \chi\left(L_{s}\right)$ is constant. Let $m$ be the maximum value of $i$ such that the function $s \mapsto \operatorname{dim} H^{i}\left(A_{s}, L_{s}\right)$ is nonzero. (We know $m \leq g$.) The constancy of $\chi\left(L_{s}\right)$ and Lemma 2.4 imply that ind $\left(L_{s}\right) \in\{m, m-2, m-4, \ldots\}$ for all $s \in S$. Since the specialization map $\phi^{m+1}(s): R^{m+1} f_{*} L \otimes k(s) \rightarrow H^{m+1}\left(A_{s}, L_{s}\right)=0$ is trivially surjective, [Hartshorne 1977, Theorem III.12.11(a)] says that it is an isomorphism for every $s \in S$; hence, $R^{m+1} f_{*} L=0$. Then Part (b) of the cited theorem implies that $\phi^{m}(s)$ is surjective for all $s \in S$. On the other hand, $\phi^{m-1}(s)$ is also trivially surjective for $s \in S$, so the same theorem shows that $R^{m} f_{*} L$ is locally free on $S$ and that $\phi^{m}(s)$ is an isomorphism. Therefore, ind $\left(L_{s}\right)=m$ for all $s \in S$, and we are done. ${ }^{2}$

Definition 2.6. A line bundle $L$ over $A$ is nondegenerate of index $i \in \mathbb{Z}$ if $\operatorname{ind}\left(L_{S}\right)=i$ for all $s \in S$.

Remark 2.7. A nondegenerate line bundle of index 0 is none other than a relatively ample line bundle.

${ }^{2}$ We refer to [Hartshorne 1977] only for convenience as it has the exact form of the theorem we need. As it is written, it applies to a (locally) projective abelian scheme $A$ over $S$. This is no problem as projectivity can be relaxed to properness by [Grothendieck 1963, III.7.7]. 
2B. Heisenberg groups. Define an $S$-subgroup scheme $K(L):=\operatorname{ker} \lambda_{L}$ of $A$. Concretely, the group $K(L)(T)$ for each $S$-scheme $T$ consists of $x \in A(T)$ such that $T_{x}^{*}(L \times T) \simeq(L \times T) \otimes p_{2}^{*} M$ for some line bundle $M$ on $S$, where $p_{2}: A \times T \rightarrow T$ is the projection map. If $L$ is nondegenerate, then $K(L)$ is a finite flat group scheme by Lemma 2.2 .

Let us define a group-valued contravariant functor $\operatorname{Aut}(L / A)$ on $(\mathrm{Sch} / S)$. The group $\operatorname{Aut}(L / A)(T)$ consists of pairs $(\psi, x)$, where $x \in A(T)$ and $\psi: L \times T \rightarrow L \times T$ is an isomorphism such that the following diagram commutes:

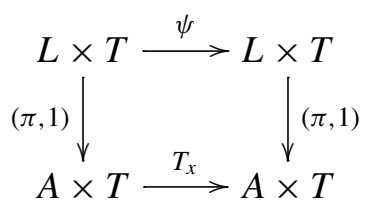

The group law is provided by $\left(\psi_{1}, x_{1}\right)\left(\psi_{2}, x_{2}\right)=\left(\psi_{1} \psi_{2}, x_{1}+x_{2}\right)$. The functor Aut $(L / A)$ is representable by a group scheme denoted $\mathscr{G}(L)$ and called a theta group or a Heisenberg group (scheme). There is a natural sequence of $S$-group schemes

$$
1 \rightarrow \mathbb{G}_{m} \rightarrow \mathscr{G}(L) \rightarrow K(L) \rightarrow 1,
$$

where the maps are respectively $\alpha \mapsto(\alpha, 0)$ and $(\psi, x) \mapsto x$ on $T$-valued points. We identified $\mathbb{G}_{m}$ with the automorphisms of $L$ over $A$. The argument in the proof of [Mumford 1974, §23, Theorem 1] shows that (2-1) is exact as Zariski sheaves. The commutator map $\mathscr{G}(L) \times \mathscr{G}(L) \rightarrow \mathscr{G}(L)$ given by $\left(\gamma_{1}, \gamma_{2}\right) \mapsto \gamma_{1} \gamma_{2} \gamma_{1}^{-1} \gamma_{2}^{-1}$ has image in $\mathbb{G}_{m}$ and induces a bilinear pairing

$$
e^{L}: K(L) \times K(L) \rightarrow \mathbb{G}_{m} .
$$

Lemma 2.8. If $L$ is nondegenerate, then $e^{L}$ is symplectic, namely alternating and nondegenerate. (The latter means that an isomorphism $K(L) \stackrel{\sim}{\rightarrow} \underline{\operatorname{Hom}}_{\mathrm{O}_{S}}\left(K(L), \mathbb{G}_{m}\right)$ is induced by $e^{L}$.)

Proof. See [Moret-Bailly 1985, IV.2.4(ii)].

2C. The Stone-von Neumann theorem and Schur's lemma. From here on we will always assume that $L$ is nondegenerate, unless it is said otherwise.

Definition 2.9 [Moret-Bailly 1985, V.1.1]. Let $G$ be a group scheme over $S$ and $\mathscr{F}_{F}$ an $\mathrm{O}_{S}$-module (always assumed to be quasicoherent). We say that $\mathscr{F}_{F}$ is a $G$ representation (on an $\mathrm{O}_{S}$-module) when $\mathscr{F}$ is equipped with a morphism of fppf sheaves in groups $G \rightarrow{\underline{\text { Aut }}}_{\mathscr{S}_{S}}(\mathscr{F})$. A morphism between two $G$-representations $\mathscr{F}_{1}$ and $\mathscr{F}_{2}$ is a morphism of $\mathrm{O}_{S}$-modules $\mathscr{F}_{1} \rightarrow \mathscr{F}_{2}$ compatible with $G$-actions. The same definition makes sense when $G$ is replaced with an fppf sheaf in groups. 
Remark 2.10. When $\underline{G}$ is a group functor on $(\mathrm{Sch} / S)$, a representation of $\underline{G}$ will mean an $\mathscr{O}_{S}$-module $\mathscr{F}$ equipped with a morphism of group functors $\underline{G} \rightarrow \underline{\text { Aut }}_{\mathscr{O}_{S}}(\mathscr{F})$,

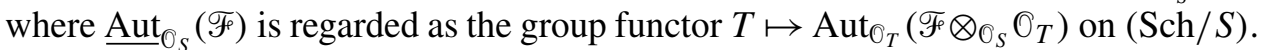

Definition 2.11 [Moret-Bailly 1985, V.2.1, V.2.3]. A $\mathscr{G}(L)$-representation $\mathscr{F}$ is of weight $w \in \mathbb{Z}$ if $\mathbb{G}_{m}$ acts on $\mathscr{F}$ via the character $\lambda \mapsto \lambda^{w}$. A $\mathscr{G}(L)$-representation $\mathscr{F}$ is irreducible if every $\mathscr{G}(L)$-subrepresentation $\mathscr{F}^{\prime}$ of $\mathscr{F}$ has the form $\mathscr{F}^{\prime}=\mathscr{F} \otimes_{\mathscr{O}_{S}} \mathscr{T}$ for some ideal sheaf $\mathscr{I}$ of $\mathcal{O}_{S}$ (equipped with trivial $\mathscr{G}(L)$-action).

Most of the time our focus will be on representations of weight 1 or -1 . The following result due to Moret-Bailly (but see Remark 2.14 below) is crucial in understanding weight-1 representations of $\mathscr{G}(L)$.

Proposition 2.12. Let $\mathscr{F}$ be a $\mathscr{G}(L)$-representation of weight 1 . Suppose that $\mathscr{F}_{F}$ is a locally free $O_{S}$-module of rank $\operatorname{deg} L$.

(i) $\mathscr{F}$ is an irreducible $\mathscr{G}(L)$-representation.

(ii) There is an equivalence between the category of $\mathrm{O}_{S}$-modules and the category of $\mathscr{G}(L)$-representations of weight 1 on $\mathrm{O}_{S}$-modules given by $\mathcal{N} \mapsto \mathscr{F} \otimes \mathcal{N}$ and $\mathcal{M} \mapsto \underline{\operatorname{Hom}}_{\varphi_{(L)}}(\mathscr{F}, \mathcal{M})$, which are canonically quasi-inverses of each other. (The composition of the two functors in any order is canonically isomorphic to the identity functor.)

(iii) If $\mathscr{F}^{\prime}$ is another weight-1 $\mathscr{G}(L)$-representation that is locally free of rank $\operatorname{deg} L$, then there exists a unique (up to isomorphism) line bundle $M$ on $S$ such that

$$
\mathscr{F}^{\prime}=\mathscr{F} \otimes M .
$$

(iv) $\operatorname{End}_{G_{(L)}}(\mathscr{F}) \simeq \mathbb{O}_{S}$ canonically.

Proof. The first two assertions are contained in [Moret-Bailly 1985, V.2.4.2, V.2.4.3]. As for (iii), clearly (ii) implies that there is an $O_{S}$-module $\mathcal{M}$ such that $\mathscr{F}^{\prime}=\mathscr{F}_{F} \otimes \mathcal{M}$. As $\mathscr{F}^{\prime}$ and $\mathscr{F}$ are locally free of the same rank, it follows that $M$ is locally free of

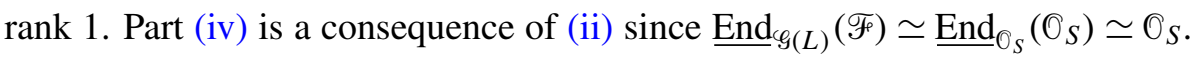

For each $0 \leq j \leq g$, note that $R^{j} f_{*} L$ is naturally a $\mathscr{G}(L)$-representation of weight 1 (which could be the zero sheaf) in the above sense. We will need the following fundamental result on $\mathscr{G}(L)$-representations:

Proposition 2.13. Assume that $S$ is locally noetherian and that $L$ has index $i$.

(i) $R^{j} f_{*} L=0$ unless $j=i$.

(ii) $R^{i} f_{*} L$ is locally free, and $\left(\operatorname{rank}_{O_{S}} R^{i} f_{*} L\right)^{2}=\operatorname{rank}_{\mathscr{O}_{S}} K(L)=(\operatorname{deg} L)^{2}$. In particular, it satisfies the condition of Proposition 2.12.

(iii) $\left(R^{i} f_{*} L\right)_{s} \simeq H^{i}\left(A_{s}, L_{s}\right)$ for each $s \in S$. 
Proof. When $i=0$, (i) and (ii) were deduced in [Mumford et al. 1994, Chapter 0, §5] from [Grothendieck 1963, III.7.7.5, III.7.7.10, III.7.8.4.]. The same results of [Grothendieck 1963] imply (i) and (ii) for arbitrary $i$. Part (iii) amounts to the assertion that $\phi^{i}(s)$ is an isomorphism as shown in the proof of Lemma 2.5.

Remark 2.14. When $S=$ Spec $k$ for an algebraically closed field $k$, the results of this subsection in this case were proved in the appendix of [Sekiguchi 1977]. (The proof is attributed to Mumford; cf. [Mumford 1966, §1].) The sheaf $R^{i} f_{*} L$ provides us with a $k$-vector space $H^{i}(A, L)$ with an action of $\mathscr{G}(L)$. Proposition 2.13 says that $H^{i}(A, L)$ is the unique (up to isomorphism) irreducible $\mathscr{G}(L)$-representation of weight 1 . When $S=\operatorname{Spec} \mathbb{C}$, Proposition 2.12 implies the classical Stone vonNeumann theorem for finite Heisenberg groups on $\mathbb{C}$-vector spaces.

2D. Matrix coefficient map. Let $\mathscr{F}$ be as in Proposition 2.12. Then

$$
\mathscr{F}^{\vee}=\underline{\operatorname{Hom}}_{\mathbb{O}_{S}}\left(\mathscr{F}, O_{S}\right)
$$

is a $\mathscr{G}(L)$-representation of weight -1 via

$$
\left(\gamma \cdot v^{\vee}\right)(v)=v^{\vee}\left(\gamma^{-1} \cdot v\right), \quad \gamma \in \mathscr{G}(L), v \in \mathscr{F}, v^{\vee} \in \mathscr{F}^{\vee} .
$$

Equip $\underline{\operatorname{Hom}}_{\mathbb{G}_{m}}\left(\mathscr{G}(L), O_{S}\right)$ with a structure of $\mathscr{G}(L) \times \mathscr{G}(L)$-representation via

$$
\left(\left(\gamma_{1}, \gamma_{2}\right) \cdot \phi\right)(\gamma)=\phi\left(\gamma_{2}^{-1} \gamma \gamma_{1}\right), \quad \gamma_{1}, \gamma_{2}, \gamma \in \mathscr{G}(L), \phi \in \underline{\operatorname{Hom}}_{\mathbb{G}_{m}}\left(\mathscr{G}(L), O_{S}\right) .
$$

Lemma 2.15. The map sending $v \otimes v^{\vee}$ to $\gamma \mapsto v^{\vee}(\gamma v)$ yields an isomorphism of $\mathscr{G}(L) \times \mathscr{G}(L)$-representations

$$
\mathscr{F} \otimes \mathscr{F}^{\vee} \stackrel{\sim}{\rightarrow} \underline{\operatorname{Hom}}_{\mathbb{G}_{m}}\left(\mathscr{G}(L), O_{S}\right) .
$$

Proof. See [Moret-Bailly 1985, Theorem V.2.4.2(i)].

\section{Adelic and $p$-adic Heisenberg groups}

In this section we consider not only a single abelian scheme $A$ but also coverings of $A$ simultaneously in order to obtain a theory of $p$-adic and adelic Heisenberg groups. Although it would be natural to deal with a tower of abelian schemes in the sense of Mumford [1967b, §7], which involves all isogenies to $A$, we have chosen to work with only multiplication-by- $n$ maps $\left(n \in \mathbb{Z}_{>0}\right)$ in favor of simplicity and concreteness. (If one wishes to make the analogue of Mumford's polarized tower of abelian schemes in our context, one may relax the ampleness condition and allow line bundles to be nondegenerate.)

Keep the notation from the previous section. In particular, $L$ is a nondegenerate line bundle over $A$. We do not assume that $L$ is symmetric until Section 3E. No condition (such as being locally noetherian) is imposed on $S$ in Section 3. 
3A. Construction of $\boldsymbol{T}$ and $\boldsymbol{V}$. This subsection is about a general construction. Let $X$ be a commutative group scheme over $S$. For each $n \in \mathbb{Z}_{>0}$, let $n: X \rightarrow X$ denote the multiplication-by- $n$ map by a slight abuse of notation. Assume that

$$
\text { for every } n \geq 1 \text {, the map } n \text { is finite and flat. }
$$

Set $X[n]:=\operatorname{ker} n$, which is a finite flat group scheme, and

$$
T X:=\underset{\leftarrow}{\lim } X[n]
$$

with respect to $m: X[m n] \rightarrow X[n]$ for each $m, n \geq 1$. Since the latter maps are finite (thus affine) $S$-morphisms, [Grothendieck 1964, IV.8.2.3] implies that the limit $T X$ exists in the category of $S$-group schemes. The underlying structure ring is $T X=\operatorname{Spec}\left(\lim _{\longrightarrow} O_{X[n]}\right)$, and as a group functor

$$
T X(T)=\left\{\left(x_{r}\right)_{r \geq 1} \mid x_{r} \in X[r](T), r x_{r s}=x_{s}, \text { if } r, s \geq 1\right\} .
$$

Define an ind-group scheme

$$
V X:=\underline{\lim } T X
$$

with respect to $m: T X \rightarrow T X$ (from the $n$-th copy of $T X$ to the $m n$-th copy for all $m, n \geq 1)$. As a group functor, for each $S$-scheme $T$,

$V X(T)=\left\{\left(x_{r}\right)_{r \geq 1} \mid x_{r} \in X(T), N x_{1}=0\right.$ for some $\left.N \geq 1, r x_{r s}=x_{s}, \forall r, s \geq 1\right\}$.

By a variant of Yoneda's lemma, $V X$ is determined as an ind group scheme by the above description as a group functor. By allowing $m$ and $n$ to run over powers of a prime $p$, we can similarly define $T_{p}$ and $V_{p}$. Note that there is a canonical isomorphism $T X \simeq \prod_{p} T_{p} X$, functorial in $X$.

There is a canonical action of $\widehat{\mathbb{Z}}$ on $T X$ coming from the compatible canonical actions of $\mathbb{Z} / r \mathbb{Z}$ on $X[r]$ for $r \geq 1$. The $\widehat{\mathbb{Z}}$-action on $T X$ patches to an action of $\mathbb{A}^{\infty}=\lim _{\rightarrow} \frac{1}{n} \widehat{\mathbb{Z}}$ on $V X$. Similarly $\mathbb{Z}_{p}$ and $\mathbb{Q}_{p}$ act on $T_{p} X$ and $V_{p} X$, respectively.

The construction of $T X, V X, T_{p} X$ and $V_{p} X$ is functorial in $X$ and carries over to commutative flat ind-group schemes $X$ satisfying $(*)$ above. For instance, $T_{p} X$ and $V_{p} X$ make sense for $p$-divisible groups $X$ over $S$.

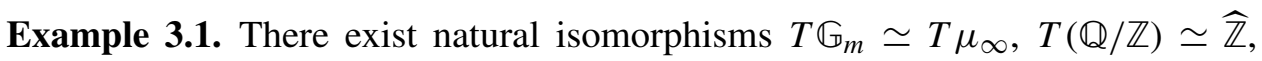
$T_{p}\left(\mathbb{Q}_{p} / \mathbb{Z}_{p}\right) \simeq \mathbb{Z}_{p}$ and $T_{p} \mathbb{G}_{m} \simeq T_{p} \mu_{p^{\infty}}$.

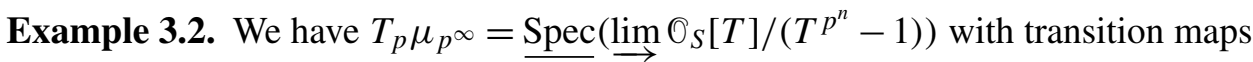
$f(T) \mapsto f\left(T^{p}\right)$.

Example 3.3. If $X$ has bounded torsion (there exists $n \geq 1$ such that $X[n]=X[\mathrm{mn}]$ for all $m \geq 1$ ), then $T X, V X, T_{p} X$ and $V_{p} X$ are all trivial group schemes. 
An important case is when $X$ is an abelian scheme. An isogeny of abelian scheme $\alpha: A^{\prime} \rightarrow A$ is said to be bounded if $\operatorname{ker} \alpha \subset A[n]$ for some $n \geq 1$. (This condition is automatic if $S$ has finitely many connected components because the fiberwise rank of ker $\alpha$ is locally constant on $S$ but not in general; suppose that $S=\bigsqcup_{j \geq 1} S_{j}$ and that for each $j, S_{j} \neq \varnothing$ and $\alpha$ is the multiplication by $j$ on $A \times{ }_{S} S_{j}$. Then $\alpha$ is not bounded.) A map of ind-group schemes $\beta: V A^{\prime} \rightarrow V A$ is said to be bounded if $m T A \subset \beta\left(n T A^{\prime}\right) \subset T A$ for some $m, n \geq 1$. The same notion is defined for a map $V_{p} \Sigma^{\prime} \rightarrow V_{p} \Sigma$, where $\Sigma^{\prime}$ and $\Sigma$ are $p$-divisible groups over $S$.

Lemma 3.4. Let $\alpha: A^{\prime} \rightarrow A$ be a bounded isogeny of abelian schemes. The induced map $V(\alpha): V A^{\prime} \rightarrow V A$ sending $\left(x_{r}\right)_{r \geq 1}$ to $\left(\alpha\left(x_{r}\right)\right)_{r \geq 1}$ is a bounded isomorphism.

Proof. We remark that the boundedness of $\alpha$ is needed to ensure that $V(\alpha)$ is an invertible map. Also note that if $\operatorname{ker} \alpha \subset A^{\prime}[m]$, then $m T A \subset V(\alpha)\left(T A^{\prime}\right) \subset T A$.

Remark 3.5. In the geometric theory of theta functions à la Mumford, one reason why $V A$ naturally shows up is that an (ample) line bundle over $A$ can be trivialized over $V A$. In this regard, $V A$ is the analogue of the universal covering spaces for complex abelian varieties. Unsurprisingly, we will see $V A$ appearing in the construction of adelic Heisenberg groups and Weil representations.

Lemma 3.6. The scheme TA is flat over $S$ and defines an fppf sheaf in groups on (Flat/S). The ind-scheme VA also defines an fppf sheaf in groups on (Flat/S). The same is true for $T_{p} A$ and $V_{p} A$.

Proof. Let us show that $T A$ is flat over $S$. We may assume that $S$ is affine. Let $S=\operatorname{Spec} C$ and $A[n]=\operatorname{Spec} B_{n}$ for $n \geq 1$. As $m: A[m n] \rightarrow A[n]$ is surjective, we see that $m^{*}: B_{n} \rightarrow B_{m n}$ is injective. Since $B_{n}$ is a flat $C$-algebra, $\lim _{\rightarrow} B_{n}$ is also one. Hence, the assertion about $T A$ follows. The fppf sheaf axiom for $V A$ is easily deduced from that for $T A$. The case of $T_{p} A$ and $V_{p} A$ is proved in the same way.

Consider a category of $p$-divisible groups over $S$ in which morphisms are bounded isogenies, and then obtain a new category by inverting bounded isogenies. When $\Sigma$ is a $p$-divisible group over $S$, let $\operatorname{Aut}_{S}^{0, \mathrm{~b}}(\Sigma)$ denote the automorphism group functor on $(\mathrm{Sch} / S)$ arising from the latter category. Let $\underline{\operatorname{Aut}}_{S}^{\mathrm{b}}\left(V_{p} \Sigma\right)$ be the group functor on $(\mathrm{Sch} / S)$ assigning bounded automorphisms. Define a map

$$
\xi: \underline{\operatorname{Aut}}_{S}^{0, \mathrm{~b}}(\Sigma) \rightarrow \underline{\operatorname{Aut}}_{S}^{\mathrm{b}}\left(V_{p} \Sigma\right)
$$

by $\alpha \mapsto\left(\left(x_{p^{r}}\right)_{r \geq 0} \mapsto\left(\alpha\left(x_{p^{r}}\right)\right)_{r \geq 0}\right)$, where $x_{1} \in \Sigma$ and $x_{p^{r}}=p x_{p^{r+1}}$.

Lemma 3.7. The above map $\xi$ is an isomorphism.

Proof. It suffices to present the inverse map $\xi^{-1}$ of $\xi$. Let $\alpha \in \underline{\operatorname{Aut}}_{S}^{\mathrm{b}}\left(V_{p} \Sigma\right)$ so that $p^{m} T_{p} \Sigma \subset p^{n} \alpha\left(T_{p} \Sigma\right) \subset T_{p} \Sigma$ for some $m \geq n \geq 0$. For each $r \geq 0, p^{n} \alpha$ maps $\left(1 / p^{r}\right) T_{p} \Sigma$ to itself, thus inducing a map $\Sigma\left[p^{r}\right] \rightarrow \Sigma\left[p^{r}\right]$ by taking quotients 
by $T_{p} \Sigma$. By patching these maps, we obtain a map $\alpha^{\prime}: \Sigma \rightarrow \Sigma$ such that ker $\alpha^{\prime}$ is killed by $p^{m}$; hence, $\alpha^{\prime}$ is bounded. Then we define $\xi^{-1}(\alpha)=p^{-n} \alpha^{\prime}$. It is routine to verify that $\xi^{-1}$ is indeed the inverse map of $\xi$.

3B. Construction of adelic and p-adic Heisenberg groups. Let $\widetilde{A}$ be the $S$-group scheme equipped with $u: \widetilde{A} \rightarrow A$, which is the inverse limit of the coverings $n: A \rightarrow A$ for all integers $n \geq 1$ (cf. [Mumford 2007, 4.27]). The limit $\widetilde{A}$ exists as a group scheme because the maps $n$ are affine, again due to [Grothendieck 1964, IV.8.2.3]. We have that $T A=\operatorname{ker} u$ in the notation of Section 3A. Set $\frac{1}{n} T A:=$ $u^{-1}(A[n])=\operatorname{ker}(n u)$. (We interpret $u^{-1}(A[n])$ as $\tilde{A} \times_{u, A} A[n]$.)

Lemma 3.8. For each $n \in \mathbb{Z}_{>0}, K\left(n^{*} L\right) \simeq A \times_{n^{2}, A} K(L)$ canonically. In other words,

$$
K\left(n^{*} L\right)(T)=\left\{x \in A(T) \mid n^{2} x \in K(L)(T)\right\} .
$$

Proof. Set $L_{T}:=L \times T$. For $x \in A(T)$,

$$
T_{x}^{*} n^{*} L_{T} \otimes\left(n^{*} L_{T}\right)^{-1} \simeq n^{*}\left(T_{n x} L_{T} \otimes L_{T}^{-1}\right) \simeq T_{n^{2} x}^{*} L_{T} \otimes L_{T}^{-1},
$$

where the second isomorphism results from the theorem of the square. Therefore, $x \in K\left(n^{*} L\right)(T)$ if and only if $n^{2} x \in K(L)(T)$.

Set $T(A, L):=u^{-1}(K(L))$ and $\frac{1}{n} T(A, L):=(n u)^{-1}(K(L))$. There are canonical identifications (as schemes over $A$ )

$$
\frac{1}{n} T A \simeq \lim _{m \geq 1} A[m n] \quad \text { and } \quad T(A, L) \simeq \lim _{m \geq 1} K\left(m^{*} L\right)
$$

We have a natural projection $u: \frac{1}{n} T A \rightarrow A[n]$. For $m, n \in \mathbb{Z}_{>0}, m: A \rightarrow A$ induces $\frac{1}{m n} T A \stackrel{\sim}{\rightarrow} \frac{1}{n} T A$. Its inverse map is denoted by $\frac{1}{m}: \frac{1}{n} T A \stackrel{\sim}{\rightarrow} \frac{1}{m n} T A$. Similarly, there are natural maps

$$
\frac{1}{n^{2}} T(A, L)=T\left(A, n^{*} L\right) \rightarrow K\left(n^{*} L\right) \quad \text { and } \quad \frac{1}{m}: \frac{1}{n} T(A, L) \stackrel{\sim}{\rightarrow} \frac{1}{m n} T(A, L) .
$$

A concrete description of $T(A, L)$ is that for each $S$-scheme $T$,

$$
T(A, L)(T)=\left\{\left(x_{r}\right)_{r \geq 1} \mid x_{r} \in A(T), x_{1} \in K(L)(T), x_{r}=s x_{r s}, \forall r, s \geq 1\right\} .
$$

The ind-group scheme $V A$ (Section 3A) is canonically identified with the ind-scheme arising from $\left\{\frac{1}{n} T A\right\}_{n \geq 1}$ with the inclusions $\frac{1}{n} T A \hookrightarrow \frac{1}{m n} T A$ for $m, n \geq 1$ as can be seen by the commutative diagram

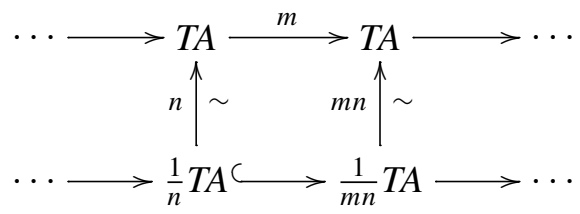


There are natural inclusions $\frac{1}{n} T A \hookrightarrow V A$ and $\frac{1}{n} T(A, L) \hookrightarrow V A$ for each $n \geq 1$.

Set $\widetilde{G}\left(n^{*} L\right):=\mathscr{G}\left(n^{*} L\right) \times_{K\left(n^{*} L\right)} \frac{1}{n^{2}} T(A, L)$. We will denote by $j_{n}$ the canonical projection $\widetilde{G}\left(n^{*} L\right) \rightarrow \frac{1}{n^{2}} T(A, L)$. The next lemma will endow us with an inclusion later. (See (3-4).)

Lemma 3.9. Let $T$ be an $S$-scheme, $x^{\prime} \in A(T)$ and $(\psi, x) \in \mathscr{G}\left(n^{*} L\right)(T)$. Suppose that $x=m x^{\prime}$. Then there exists a unique $\psi^{\prime}:(m n)^{*} L \stackrel{\sim}{\rightarrow}(m n)^{*} L$ such that $\left(\psi^{\prime}, x^{\prime}\right) \in \mathscr{G}\left((m n)^{*} L\right)(T)$ and

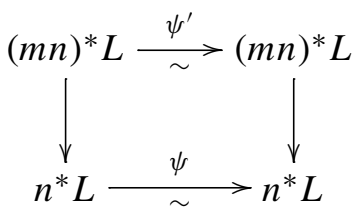

commutes, where the vertical maps are the projection maps $\left(\right.$ as $(m n)^{*} L$ is the fiber product of $n^{*} L$ with A over $m: A \rightarrow A$ ).

Proof. Without loss of generality, we may assume that $n=1$. The uniqueness is easy. If $\left(\psi^{\prime}, x^{\prime}\right),\left(\psi^{\prime \prime}, x^{\prime}\right) \in \mathscr{G}\left(m^{*} L\right)(T)$ have the property as above, then the difference $\left(\psi^{\prime \prime}\left(\psi^{\prime}\right)^{-1}, 0\right)$ is in the image of some $t \in \mathbb{G}_{m}(T)$ under $\mathbb{G}_{m} \rightarrow \mathscr{G}(L)$. This means that (3-2) remains commutative after multiplying $t$ to the top arrow. This implies that $t=1$ and $\psi^{\prime}=\psi^{\prime \prime}$.

Let us verify the existence of $\psi^{\prime}$. The fact that $(\psi, x) \in \mathscr{G}(L)(T)$ induces an isomorphism $\xi: L \stackrel{\sim}{\rightarrow} T_{x}^{*} L$ making the top triangle in the left diagram commute. In the following two diagrams, the rectangles are cartesian squares. (We are abusing the notation to use $A$ and $L$ to denote $A \times{ }_{S} T$ and $L \times{ }_{S} T$.)
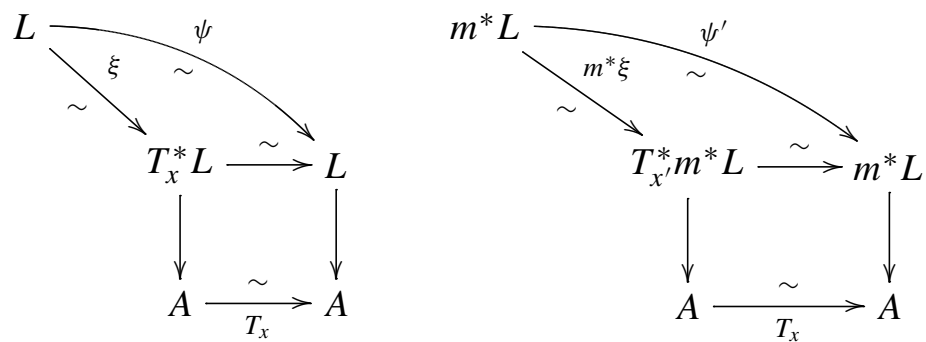

Then $m^{*} L \stackrel{m^{*} \xi}{\simeq} m^{*} T_{x}^{*} L \simeq T_{x^{\prime}}^{*} m^{*} L$. (The latter holds because $m \circ T_{x^{\prime}}=T_{x} \circ m$.) Let $\psi^{\prime} \in \underline{\operatorname{Aut}}\left(m^{*} L\right)(T)$ be the latter map composed with $T_{x^{\prime}}^{*} m^{*} L \simeq m^{*} L$ in the above diagram. Then $\left(\psi^{\prime}, x^{\prime}\right) \in \mathscr{G}\left(m^{*} L\right)(T)$, so (3-2) commutes up to an automorphism of $L$ fixing $L \rightarrow A$. Such an automorphism is a multiplication by $s \in \mathbb{O}_{T}^{\times}$. The commutativity of (3-2) is achieved by multiplying $t$ to $\psi^{\prime}$.

Remark 3.10. (This remark is to be recalled in the proof of Lemma 4.5.) By associating $\psi^{\prime}$ to $x^{\prime} \in A[m](T)$ with $x=0$ and $\psi=$ id in Lemma 3.9, we can define 
an action of $A[m]$ on $(m n)^{*} L$. This action is the same as the $A[m]$-action induced on $(m n)^{*} L$ via Proposition 4.1 (by taking $G=A[m]$ and $\xi$ to be $n: A \rightarrow A$ ). This can be seen from the fact that the quotient of $(m n)^{*} L$ with respect to the former $A[m]$-action is $n^{*} L$, as shown in the proof of Lemma 3.15.

Corollary 3.11. For each $S$-scheme $T$, the set $\widetilde{G}\left(n^{*} L\right)(T)$ may be described as the set of $\left(\psi_{r}, x_{r}\right)_{r \geq 1}$ such that

(i) $\left(\psi_{r}, x_{r}\right) \in \mathscr{G}\left((r n)^{*} L\right)(T)$ for all $r \geq 1$,

(ii) $x_{r}=s x_{r s}$ for all $r, s \geq 1$ and

(iii) the following diagram commutes for all $r, s \geq 1$ :

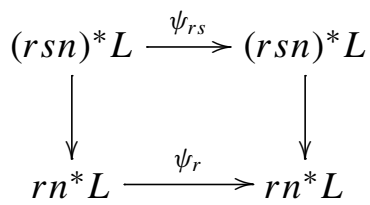

Proof. The set of $\left(\psi_{r}, x_{r}\right)_{r \geq 1}$ in the corollary will be temporarily called $\widetilde{\mathscr{G}}_{0}\left(n^{*} L\right)(T)$. As $\widetilde{\mathscr{G}}\left(n^{*} L\right)=\mathscr{G}\left(n^{*} L\right) \times_{K\left(n^{*} L\right)} \frac{1}{n^{2}} T(A, L)$, we see that $\widetilde{G}\left(n^{*} L\right)(T)$ consists of $\psi_{1}$ and $\left(x_{r}\right)_{r \geq 1}$ such that $\left(\psi_{1}, x_{1}\right) \in \mathscr{G}\left(n^{*} L\right)(T)$ and $x_{r}=s x_{r s}$ for all $r, s \geq 1$.

There is an obvious map

$$
\widetilde{\mathscr{G}}_{0}\left(n^{*} L\right)(T) \rightarrow \widetilde{\mathscr{G}}\left(n^{*} L\right)(T)
$$

forgetting $\psi_{r}$ for $r \geq 2$. By Lemma 3.9, for a choice of $\psi_{1}$ and $\left(x_{r}\right)_{r \geq 1}$, there exists a sequence $\left(\psi_{r}\right)_{r \geq 2}$ satisfying (iii) of the corollary, and such a sequence is unique. Therefore, (3-3) is a bijection.

Henceforth, $\widetilde{G}\left(n^{*} L\right)$ is viewed as the $S$-group scheme whose associated group functor is described as in Corollary 3.11. It is flat over $S$ and defines an fppf sheaf on $S$ by essentially the same argument as in the proof of Lemma 3.6. Thanks to Lemma 3.9, we have a map of group schemes

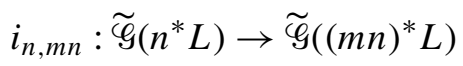

sending $\left(\left(\psi_{r}, x_{r}\right)_{r \geq 1}\right) \mapsto\left(\left(\psi_{r}^{\prime}, x_{r}^{\prime}\right)_{r \geq 1}\right)$ on $T$-valued points, where $x_{r}^{\prime}=x_{r m}$ and $\psi_{r}^{\prime}=\psi_{r m}$.

Lemma 3.12. The following diagram commutes, and its rows are fppf exact:

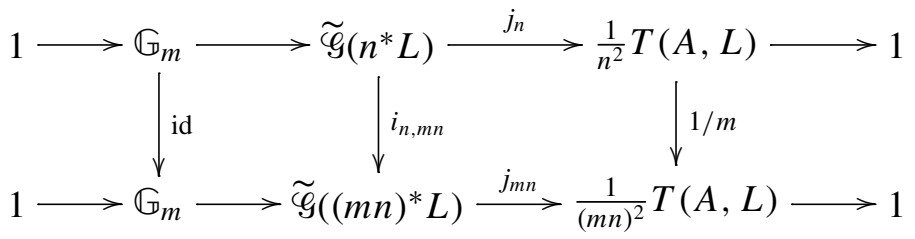


Proof. Everything is obvious except perhaps the surjectivity. The map $j_{n}$ is fppf surjective as it is a base change of the fppf surjective map $\mathscr{G}\left(n^{*} L\right) \rightarrow K\left(n^{*} L\right)$ (cf. Section 2B). Similarly, $j_{m n}$ is fppf surjective.

Let us define an ind-group scheme via $i_{n, m n}$ :

$$
\widehat{\mathscr{G}}(L):=\underset{n}{\lim } \widetilde{\mathscr{G}}\left(n^{*} L\right) .
$$

Under the limit, the maps $n j_{n}: \widetilde{\mathscr{G}}\left(n^{*} L\right) \rightarrow \frac{1}{n} T(A, L)$ induce a map $\hat{j}: \widehat{\mathscr{G}}(L) \rightarrow V A$. The fact that $\widetilde{\mathscr{G}}\left(n^{*} L\right)$ are fppf sheaves shows that $\widehat{\mathscr{G}}(L)$ is also one. We have a commutative diagram where rows are fppf exact sequences:

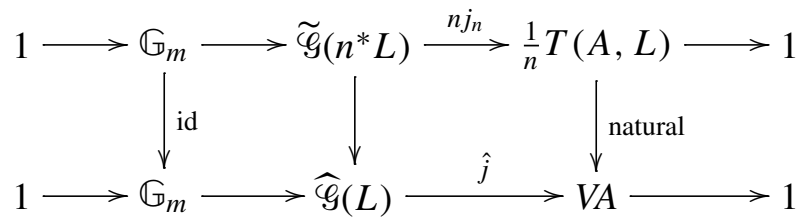

Lemma 3.13. Let $A^{\prime}$ be an abelian scheme over $S$ and $\alpha: A^{\prime} \rightarrow A$ a bounded isogeny. Then $\alpha$ induces an isomorphism $\widehat{\mathscr{G}}(\alpha): \widehat{\mathscr{G}}\left(\alpha^{*} L\right) \rightarrow \widehat{\mathscr{G}}(L)$ fitting in the commutative diagram below:

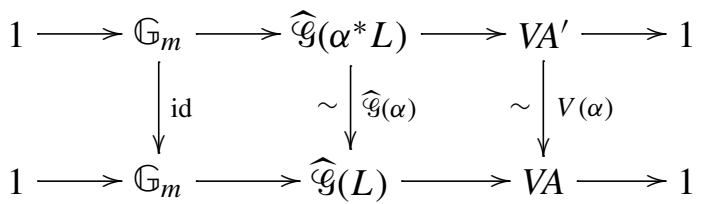

Proof. The map $\widehat{\mathscr{G}}(\alpha)$ comes from the maps $\widetilde{\mathscr{G}}\left(\alpha^{*} n^{*} L\right) \rightarrow \widetilde{\mathscr{G}}\left(n^{*} L\right)$ for $n \geq 1$, which are constructed as $\left(\psi, x_{r}\right)_{r \geq 1} \mapsto\left(\phi, \alpha\left(x_{r}\right)\right)_{r \geq 1}$. Here $\phi: L \stackrel{\sim}{\rightarrow} L$ is obtained from $\widetilde{\mathscr{G}}\left(\alpha^{*} n^{*} L\right)$ by taking the quotient of the diagram below by the action of $\operatorname{ker} \alpha$ :

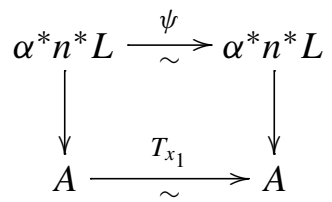

It is straightforward to verify that $\widehat{\mathscr{G}}(\alpha)$ is compatible with the maps id and $V(\alpha)$ and thus an isomorphism.

Now let $L^{\prime}$ be a line bundle over $A^{\prime}$ such that $L^{\prime} \simeq \alpha^{*} L$. This induces an isomorphism $\widehat{\mathscr{G}}\left(L^{\prime}\right) \simeq \widehat{\mathscr{G}}\left(\alpha^{*} L\right)$. It is easy to check that the latter isomorphism is independent of the choice of the isomorphism $L^{\prime} \simeq \alpha^{*} L$. By composing with $\widehat{\mathscr{G}}(\alpha)$, we obtain an isomorphism $\widehat{\mathscr{G}}\left(L^{\prime}\right) \simeq \widehat{\mathscr{G}}(L)$. 
Lemma 3.14. Let $A, A^{\prime}, \alpha, L$ and $L^{\prime}$ be as above. The isomorphism $\widehat{\mathscr{G}}\left(L^{\prime}\right) \simeq \widehat{\mathscr{G}}(L)$ fits into the following commutative diagram:

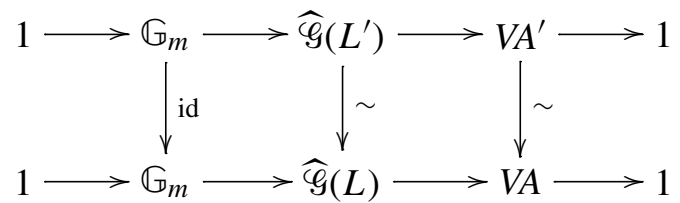

Proof. In view of Lemma 3.13, it is enough to note the obvious commutativity:

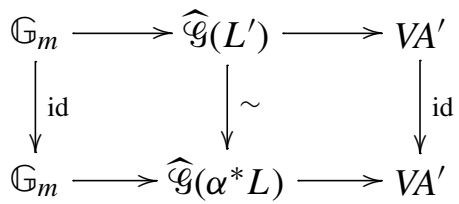

3C. The map $\hat{\sigma}$. Define $\sigma_{1}: T A \rightarrow \mathscr{G}(L)$ by $\left(x_{r}\right)_{r \geq 1} \mapsto\left(\right.$ id, $\left.x_{1}\right)$ and

$$
\widetilde{\sigma}_{1}: T A \rightarrow \widetilde{\mathscr{G}}(L)=\mathscr{G}(L) \times_{K(L)} T(A, L)
$$

by $\sigma_{1}$ and the natural inclusion $T A \hookrightarrow T(A, L)$. For $n>1$, set $\widetilde{\sigma}_{n}:=i_{1, n} \circ \widetilde{\sigma}_{1}$. Further composing with the projection $\widetilde{\mathscr{G}}\left(n^{*} L\right) \rightarrow \mathscr{G}\left(n^{*} L\right)$, we obtain $\sigma_{n}: T A \rightarrow \mathscr{G}\left(n^{*} L\right)$. By construction, $\widetilde{\sigma}_{n} \mathrm{~s}$ are compatible with the inclusions $i_{n, m n}$ for $m, n \geq 1$ and thus yield a map $\hat{\sigma}: T A \rightarrow \widehat{\mathscr{G}}(L)$. Note that $\widetilde{\sigma}_{n}, \sigma_{n}(n \geq 1)$ and $\hat{\sigma}$ are morphisms of (ind-)group schemes and that $\mathbb{G}_{m} \cap \hat{\sigma}(n T A)=\{1\}$ in $\widehat{\mathscr{G}}(L)$ for every $n \geq 1$.

Lemma 3.15 [Mumford 2007, Proposition 4.13].

(i) $N_{\widehat{\mathscr{G}}(L)}(\hat{\sigma}(n T A))=Z_{\widehat{G}_{(L)}}(\hat{\sigma}(n T A))$.

(ii) $Z_{\widehat{\mathscr{G}}_{(L)}}(\hat{\sigma}(n T A)) \simeq \widetilde{\mathscr{G}}\left(n^{*} L\right)$ canonically.

(iii) There is an isomorphism $N_{\widehat{G}}(\hat{\sigma}(n T A)) / \hat{\sigma}(n T A) \stackrel{\sim}{\rightarrow} \mathscr{G}_{\left(n^{*} L\right)}$ induced by the canonical maps

$$
N_{\widehat{G}(L)}(\hat{\sigma}(n T A)) \simeq \widetilde{\mathscr{G}}\left(n^{*} L\right) \rightarrow \mathscr{G}\left(n^{*} L\right) .
$$

Proof. As usual, we implicitly work on $T$-points for some $S$-scheme $T$. Let $X=\left(\phi_{r}, x_{r}\right)_{r \geq 1} \in N_{\widehat{G}_{(L)}}(\hat{\sigma}(n T A))$ and $Y=\left(\psi_{r}, y_{r}\right)_{r \geq 1} \in \hat{\sigma}(n T A)$. Part (i) follows from the fact that

$$
X Y X^{-1} Y^{-1} \in \mathbb{G}_{m} \cap \hat{\sigma}(n T A)=\{1\} .
$$

Let us prove (ii). For some $m \geq 1,\left(\phi_{r}^{\prime}, x_{r}^{\prime}\right)_{r \geq 1} \in \widetilde{\mathscr{G}}\left((m n)^{*} L\right)$ may represent an element of $\widehat{\mathscr{G}}(L)$. It suffices to show that if $\left(\phi_{r}^{\prime}, x_{r}^{\prime}\right)_{r \geq 1}$ centralizes $\hat{\sigma}(n T A)$, then $\left(\phi_{r}^{\prime}, x_{r}^{\prime}\right)_{r \geq 1}=i_{n, m n}\left(\left(\phi_{r}, x_{r}\right)_{r \geq 1}\right)$ for some $\left(\phi_{r}, x_{r}\right)_{r \geq 1} \in \widetilde{G}_{(}\left(n^{*} L\right)$. Consider the 
commutative diagram

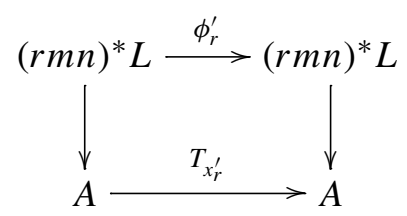

The image of $\hat{\sigma}(n T A)$ in $\widetilde{G}\left((m n)^{*} L\right)$ is none other than $\widetilde{\sigma}_{m n}(n T A)$, which consists of $\left(\psi_{r}, y_{r}\right)_{r \geq 1}$ such that $y_{r} \in A[r m]$. Recall that $A[m]$ acts on $(r m n)^{*} L$ as explained at the beginning of Remark 3.10. Let us verify that the whole diagram (3-6) is $A[m]$ equivariant. (In fact it is even $A[r m]$-equivariant.) Since $\left(\phi_{r}^{\prime}, x_{r}^{\prime}\right)_{r \geq 1}$ commutes with elements of $\widetilde{\sigma}_{m n}(n T A)$, the top arrow in the diagram is $A[m]$-equivariant. The vertical maps are $A[m]$-equivariant by [Mumford 2007, Lemma 4.11]. The same fact is obvious for the bottom map. By taking quotients of (3-6) by $A[m]$, we obtain $\phi_{r}$ such that the following commutes:

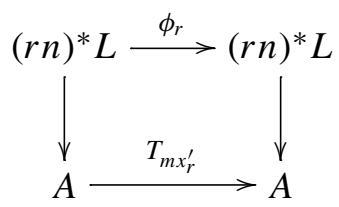

By Lemma 3.9, $\left(\phi_{r}^{\prime}, x_{r}^{\prime}\right)_{r \geq 1}=i_{n, m n}\left(\left(\phi_{r}, m x_{r}^{\prime}\right)_{r \geq 1}\right)$. The proof of (ii) is complete.

For the proof of (iii), it is enough to note that the image of $\hat{\sigma}(n T A)$ in $\widetilde{\mathscr{G}}_{(}\left(n^{*} L\right)$ consists of $\left(\phi_{r}, x_{r}\right)_{r \geq 1}$ such that $\left(\phi_{1}, x_{1}\right)$ is the identity element.

Lemma 3.16. Let $(A, L)$ be as before, $\alpha: A^{\prime} \rightarrow A$ be a bounded isogeny and $L^{\prime}=\alpha^{*} L$. Let $\hat{\sigma}^{\prime}: T A^{\prime} \rightarrow \widehat{G}\left(L^{\prime}\right)$ denote the analogue of $\hat{\sigma}$ constructed from $\left(A^{\prime}, L^{\prime}\right)$. Then the following commutes:

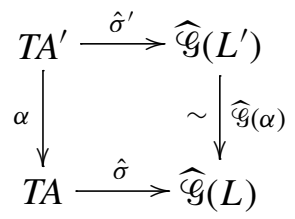

Proof. Let $\left(x_{r}\right)_{r \geq 1} \in T A^{\prime}$. Both $\widehat{\mathscr{G}}(\alpha) \circ \hat{\sigma}^{\prime}$ and $\hat{\sigma} \circ \alpha$ map $\left(x_{r}\right)_{r \geq 1}$ to (id, $\alpha\left(\left(x_{r}\right)_{r \geq 1}\right)$ ) in $\widetilde{G}(L)$.

3D. The pairing $\hat{\boldsymbol{e}}^{L}$. In analogy with $e^{L}$ in Section $2 \mathrm{~B}$, we obtain a bilinear commutator pairing from the bottom row of (3-5),

$$
\hat{e}^{L}: V A \times V A \rightarrow \mathbb{G}_{m},
$$


which is a morphism of ind-group schemes over $S$. On the other hand, the $\mathbb{Z} / n \mathbb{Z}$ linear Weil pairings

$$
e_{n}^{L, \text { Weil }}: A[n] \times A[n] \rightarrow \mu_{n} \hookrightarrow \mathbb{G}_{m}
$$

for $n \geq 1$ are glued to an $\mathbb{A}^{\infty}$-linear pairing (cf. Section 3A)

$$
\hat{e}^{L, \text { Weil }}: V A \times V A \rightarrow V \mathbb{G}_{m} .
$$

Concretely on the functors of points, the map is

$$
\left(\left(x_{n}\right)_{n \geq 1},\left(y_{n}\right)_{n \geq 1}\right) \mapsto\left(e_{N^{2} n}^{L, \text { Weil }}\left(x_{N n}, y_{N n}\right)\right)_{n \geq 1},
$$

where $N \geq 1$ is such that $x_{1}, y_{1} \in A\left[N^{2}\right]$. The definition is independent of $N$. The right side is an element of $V \mathbb{G}_{m}$ since $e_{N^{2} m n}^{L \text {,Weil }}\left(x_{N m n}, y_{N m n}\right)^{m}=e_{N^{2} n}^{L \text {, Weil }}\left(x_{N n}, y_{N n}\right)$ for any $m, n \geq 1$. Let $b: V \mathbb{G}_{m} \rightarrow \mathbb{G}_{m}$ be the map $b\left(\left(x_{r}\right)_{r \geq 1}\right)=x_{1}$ in the notation of (3-1).

Lemma 3.17. The pairing $\hat{e}^{L}$ is nondegenerate, and $\hat{e}^{L}=b \circ \hat{e}^{L \text {, Weil }}$.

Proof. The nondegeneracy of $\hat{e}^{L}$ is deduced from the nondegeneracy of $e^{n^{*} L}$ for all $n \geq 1$ (Lemma 2.8). Indeed, if $\hat{e}^{L}$ were degenerate, there would be an $S$-scheme $T$ and a nonzero section $x \in \frac{1}{n} T(A, L)(T)$ such that $\hat{e}^{L}(x, y)=1$ for any section $y$ of $V A$ in a $T$-scheme. Choose a large enough $m \geq 1$ such that $x \notin \hat{\sigma}(m n T A)$. Then $x$ has nontrivial image $\bar{x}$ in $\frac{1}{m n} T(A, L) / \hat{\sigma}(m n T A) \simeq K\left((m n)^{*} L\right)$, but by the assumption, $\bar{x}$ pairs trivially with any section of $K\left((m n)^{*} L\right)$ via $e^{(m n)^{*} L}$. This contradicts the nondegeneracy of $e^{(m n)^{*} L}$.

Let us now prove the second assertion. Let $(\phi, x),(\psi, y) \in \widehat{\mathscr{G}}(L)$, and write $x=\left(x_{n}\right)_{n \geq 1}, y=\left(y_{n}\right)_{n \geq 1} \in V A$. For any $N \geq 1$ chosen as above,

$$
b\left(\hat{e}^{L, \text { Weil }}\left(\left(x_{n}\right)_{n \geq 1},\left(y_{n}\right)_{n \geq 1}\right)\right)=e_{N^{2} n}^{L \text {, Weil }}\left(x_{N n}, y_{N n}\right)=e^{L}\left(x_{N}, y_{N}\right)^{N^{2}}=e^{L}\left(x_{1}, y_{1}\right) .
$$

(The second equality is standard. See Property (5) of [Mumford 1974, §23] for instance.) Consider the following commutative diagram:

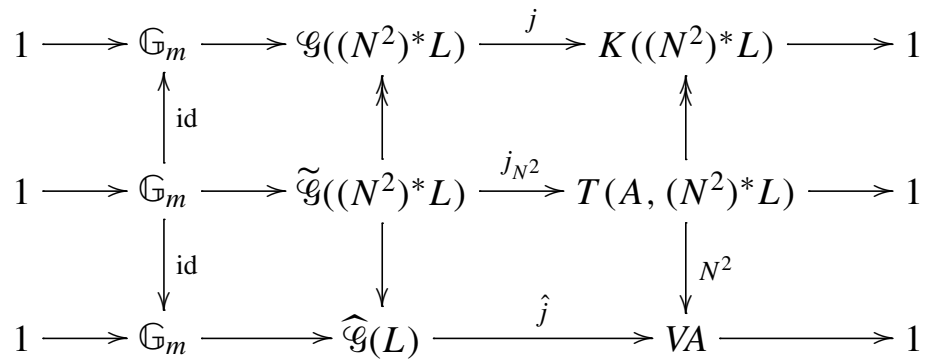

Let $x^{\prime}=\left(x_{n}^{\prime}\right)_{n \geq 1}$ and $y^{\prime}=\left(y_{n}^{\prime}\right)_{n \geq 1}$ be such that $x_{n}^{\prime}=x_{N^{2} n}$ and $y_{n}^{\prime}=y_{N^{2} n}$. Note that $x_{1}^{\prime}, y_{1}^{\prime} \in A\left[N^{4}\right] \subset K\left(\left(N^{2}\right)^{*} L\right)$; thus, $x^{\prime}, y^{\prime} \in T\left(A,\left(N^{2}\right)^{*} L\right)$. The commutativity of 
the diagram allows us to equalize the commutator pairing for each row. The second assertion follows from

$$
\hat{e}^{L}(x, y)=\phi \psi \phi^{-1} \psi^{-1}=e^{\left(N^{2}\right)^{*} L}\left(x_{1}^{\prime}, y_{1}^{\prime}\right)=e^{L}\left(N x_{1}^{\prime}, N y_{1}^{\prime}\right)=e^{L}\left(x_{1}, y_{1}\right) .
$$

3E. Symmetric line bundles and the map $\hat{\boldsymbol{\tau}}$. Our construction of $\hat{\tau}$ is based on [Mumford 2007, §4] as well as Step V in Appendix I of that book. From here on, assume that $L$ is symmetric, i.e., $(-1)^{*} L \simeq L$. There is an isomorphism (e.g., appeal to Lemma 3.14 with $A=A^{\prime}, \alpha=-1$ and $L^{\prime}=L$ )

$$
\widehat{\mathscr{G}}\left((-1)^{*} L\right) \stackrel{\sim}{\rightarrow} \widehat{\mathscr{G}}(L)
$$

uniquely characterized as follows. If $\left(\phi_{r}, x_{r}\right)_{r \geq 1}$ is mapped to $\left(\psi_{r},-x_{r}\right)_{r \geq 1}$, then the diagram below commutes, where the vertical maps are induced by the pullback along $(-1): A \rightarrow A$ :

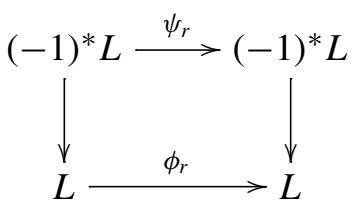

A choice of an isomorphism $I: L \simeq(-1)^{*} L$ induces $\widehat{\mathscr{G}}(L) \stackrel{\sim}{\rightarrow} \widehat{\mathscr{G}}\left((-1)^{*} L\right)$. By composing with (3-7), we obtain an isomorphism

$$
i^{L}: \widehat{\mathscr{G}}(L) \stackrel{\sim}{\rightarrow} \widehat{\mathscr{G}}(L)
$$

and can show that it is independent of the choice of $I$ (cf. [Mumford 2007, Proposition 4.16]). The situation may be understood through a commutative diagram:

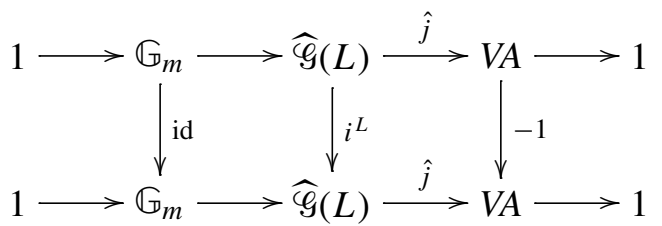

For each $n \geq 1$, clearly the map $x \mapsto x i^{L}(x)^{-1}$ from $\widetilde{\mathscr{G}}\left(n^{*} L\right)$ to $\widetilde{\mathscr{G}}\left(n^{*} L\right)$ factors as the composite of $j_{n}: \widetilde{\mathscr{G}}\left(n^{*} L\right) \rightarrow \frac{1}{n^{2}} T(A, L)$ and $h_{n}: \frac{1}{n^{2}} T(A, L) \rightarrow \widetilde{\mathscr{G}}\left(n^{*} L\right)$. We construct $\widetilde{\tau}_{2 n}$ as the composite

$$
\frac{1}{2 n^{2}} T(A, L) \stackrel{\frac{1}{2}}{\rightarrow} \frac{1}{(2 n)^{2}} T(A, L) \stackrel{h_{2 n}}{\rightarrow} \widetilde{\mathscr{G}}\left((2 n)^{*} L\right) .
$$

When $n$ is odd, $\widetilde{\tau}_{n}: \frac{1}{n^{2}} T(A, L) \rightarrow \widetilde{\mathscr{G}}\left(n^{*} L\right)$ is defined as

$$
\frac{1}{n^{2}} T(A, L) \stackrel{\frac{1}{2}}{\simeq} \frac{1}{n^{2}} T(A, L) \stackrel{h_{n}}{\rightarrow} \widetilde{\mathscr{G}}\left(n^{*} L\right) .
$$


It is readily checked that $\widetilde{\tau}_{n}$ are compatible with $i_{n, m n}$ for $m, n \geq 1$ so that they glue together to a map $\hat{\tau}: V A \rightarrow \widehat{\mathscr{G}}(L)$. (Note that $\widetilde{\tau}_{n}$ and $\widetilde{\tau}_{m n}$ provide sections in the diagram of Lemma 3.12.) By construction, $\hat{\tau}$ is a section of $\hat{j}$, namely

$$
\hat{j} \circ \hat{\tau}=\mathrm{id} \text {. }
$$

The map $\hat{\tau}$ enables us to identify $\widehat{\mathscr{G}}(L)$ with $\mathbb{G}_{m} \times V A$ equipped with a certain group law that resembles the classical Heisenberg group law. To be precise, define a group law on $\mathbb{G}_{m} \times V A$ by

$$
(\lambda, x) \cdot(\mu, y)=\left(\lambda \mu \cdot \hat{e}^{L}\left(\frac{1}{2} x, y\right), x+y\right) .
$$

Lemma 3.18 [Mumford 2007, Proposition 4.18.B]. The map

$$
\mathbb{G}_{m} \times V A \rightarrow \widehat{\mathscr{G}}(L), \quad(\lambda, x) \mapsto \lambda \cdot \hat{\tau}(x)
$$

is an isomorphism of ind-group schemes over $S$.

Proof. The above map is readily seen to be an isomorphism of ind-schemes over $S$ from the row exactness of (3-8) together with (3-9). It remains to check the homomorphism property. Set $\tilde{x}=\hat{\tau}(x / 2)$ and $\tilde{y}=\hat{\tau}(y / 2)$. Let $\lambda, \mu \in \mathbb{G}_{m}$. Then

$$
\begin{aligned}
\lambda \hat{\tau}(x) \mu \hat{\tau}(y) & =\lambda \mu \hat{\tau}(x) \hat{\tau}(y) \\
& =\lambda \mu \tilde{x} i^{L}(\tilde{x})^{-1} \tilde{y} i^{L}(\tilde{y})^{-1} \\
& =\lambda \mu \tilde{x} i^{L}(\tilde{x})^{-1} \tilde{y}\left(\tilde{x} i^{L}(\tilde{x})^{-1} \tilde{y}\right)^{-1} \tilde{y} \tilde{x} i^{L}(\tilde{x})^{-1} i^{L}(\tilde{y})^{-1} \\
& =\lambda \mu \hat{e}^{L}\left(\hat{j}\left(\tilde{x} i^{L}(\tilde{x})^{-1}\right), \hat{j}(\tilde{y})\right) \tilde{y} \tilde{x} i^{L}\left((\tilde{y} \tilde{x})^{-1}\right) \\
& =\lambda \mu \hat{e}^{L}(x, y / 2) \hat{\tau}(\tilde{x}+\tilde{y})=\lambda \mu \hat{e}^{L}(x / 2, y) \hat{\tau}(\tilde{x}+\tilde{y}) .
\end{aligned}
$$

It is natural to ask about the difference between $\hat{\sigma}$ and $\left.\hat{\tau}\right|_{T A}$, which are maps from $T A$ to $\widehat{G}(L)$. Consider the map

$$
e_{*}^{L}: \frac{1}{2} T A \rightarrow \mathbb{G}_{m}, \quad e_{*}^{L}(x)=\hat{\sigma}^{L}(2 x) \hat{\tau}(2 x)^{-1} .
$$

Lemma 3.19. The map $e_{*}^{L}$ is a quadratic form factoring as

$$
\frac{1}{2} T A \rightarrow A[2] \rightarrow \mu_{2} \hookrightarrow \mathbb{G}_{m},
$$

where $\frac{1}{2} T A \rightarrow A[2]$ and $\mu_{2} \hookrightarrow \mathbb{G}_{m}$ are canonical surjection and injection. In particular, $\hat{\sigma}$ and $\hat{\tau}$ coincide on $2 \cdot$ TA. For all $x, y \in \frac{1}{2} T A$ (viewed as $T$-valued points for each $S$-scheme $T$ ),

$$
e_{*}^{L}(x+y) e_{*}^{L}(x)^{-1} e_{*}^{L}(y)^{-1}=e^{L}(x, y)^{2} .
$$

Proof. The proof of [Mumford 2007, Proposition 4.18.C] is easily adapted to the scheme-theoretic setting as in the proof of the last lemma. 
Lemma 3.20. Consider $\left(A^{\prime}, L^{\prime}\right)$ and $(A, L)$ with a bounded isogeny $\alpha: A^{\prime} \rightarrow A$ such that $L^{\prime}=\alpha^{*} L$. Suppose that $L^{\prime}$ and $L$ are symmetric. Then the following diagram commutes, where the row isomorphisms are as in Lemma 3.18:

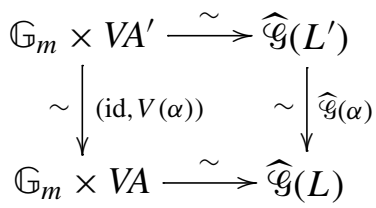

Proof. The proof is reduced to checking $i^{L} \circ \widehat{\mathscr{G}}(\alpha)=\widehat{\mathscr{G}}(\alpha) \circ i^{L^{\prime}}$, which amounts to the commutativity of the outer rectangle below:

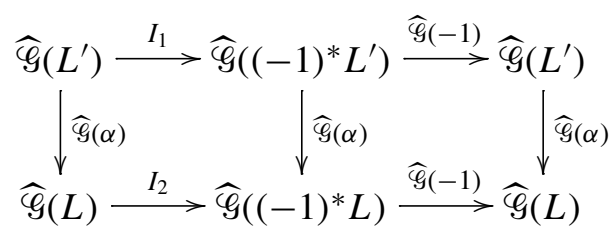

The maps $I_{1}$ and $I_{2}$ are induced by any choice of isomorphisms $L^{\prime} \simeq(-1)^{*} L^{\prime}$ and $L \simeq(-1)^{*} L$ (since such isomorphisms allow us to make the identifications $\underline{\operatorname{Aut}}\left(L^{\prime} / A\right) \simeq \underline{\operatorname{Aut}}\left((-1)^{*} L^{\prime} / A\right)$ and $\left.\underline{\operatorname{Aut}}\left(L^{\prime} / A\right) \simeq \underline{\operatorname{Aut}}\left((-1)^{*} L^{\prime} / A\right)\right)$, and they are easily seen to be independent of the choice. The right half commutes because $\widehat{\mathscr{G}}(\alpha) \widehat{\mathscr{G}}(-1)=\widehat{\mathscr{G}}(-1) \widehat{\mathscr{G}}(\alpha)=\widehat{\mathscr{G}}(-\alpha)$. In order to verify that the left half commutes, one reduces to the situation where $\widehat{\mathscr{G}}, L^{\prime}$ and $L$ are replaced with $\widetilde{\mathscr{G}}, n^{*} L^{\prime}$ and $n^{*} L$, respectively. Then by using the description of Corollary 3.11 , one checks that

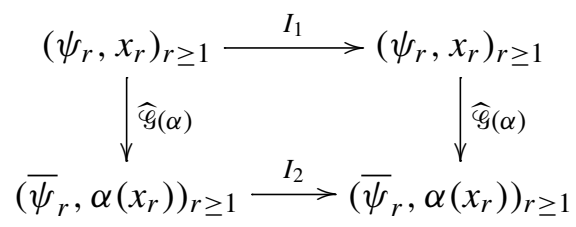

where $\bar{\psi}_{r}$ is the induced automorphism of $L \rightarrow A$ obtained from taking quotient by ker $\alpha$ of $\psi_{r}$. (The latter is an automorphism of $L^{\prime} \rightarrow A^{\prime}$.)

3F. p-adic Heisenberg groups. It is easy to adapt the construction of this section to obtain $p$-adic analogues. There are obvious definitions of $\frac{1}{p^{n}} T_{p}(A, L)$ and $\widetilde{\mathscr{G}}_{p}\left(\left(p^{n}\right)^{*} L\right)$. In order that $T_{p}(A, L)$ be contained in $V_{p} A$, we need to assume that $\operatorname{deg} L$ is a power of $p$ (including $\operatorname{deg} L=1$ ) or equivalently that $K(L)$ is a $p$-group. Then we have $\frac{1}{p^{n}} T_{p}(A, L) \hookrightarrow V_{p} A$ for all $n \geq 1$. Define

$$
\widehat{\mathscr{G}}_{p}(L):=\underset{n}{\lim } \widetilde{\mathscr{G}}_{p}\left(\left(p^{n}\right)^{*} L\right) .
$$


There is a commutative diagram similar to (3-5):

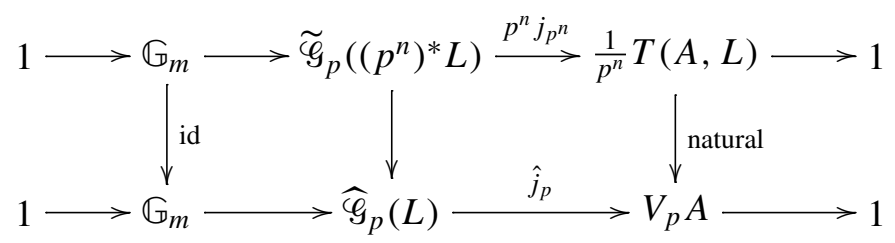

The commutator pairing yields $\hat{e}_{p}^{L}: V_{p} A \times V_{p} A \rightarrow \mathbb{G}_{m}$. A group morphism $\hat{\sigma}_{p}: T_{p} A \rightarrow \widehat{\mathscr{G}}_{p}(L)$ is constructed as before. Now suppose that $L$ is symmetric. Then there is a map $\hat{\tau}_{p}: V_{p} A \rightarrow \widehat{G}_{p}(L)$ (which is not compatible with group structure) such that $\hat{j}_{p} \circ \hat{\tau}_{p}=$ id. If $p \neq 2$, then $\hat{\sigma}_{p}^{L}=\left.\hat{\tau}_{p}\right|_{T_{p} A}$ and $e_{p, *}^{L} \equiv 1$. If $p=2$, the map $e_{2, *}^{L}: \frac{1}{2} T_{2} A \rightarrow \mathbb{G}_{m}$ sending $x$ to $\hat{\sigma}_{2}^{L}(2 x) \hat{\tau}_{2}(2 x)^{-1}$ factors through $\frac{1}{2} T_{2} A \rightarrow A[2] \rightarrow \mu_{2} \hookrightarrow \mathbb{G}_{m}$ and satisfies the same formula as in Lemma 3.19. Using $\hat{\tau}_{p}$ we get an isomorphism $\mathbb{G}_{m} \times V_{p} A \stackrel{\sim}{\rightarrow} \widehat{\mathscr{G}}_{p}(L)$ (for any $p$ including $p=2$ ) if the group law on the left-hand side is as in (3-10).

\section{Adelic and $p$-adic Heisenberg representations}

As before, $A$ is an abelian scheme over $S$, and $L$ is a nondegenerate line bundle over $A$. Throughout Section $4, S$ is locally noetherian, but $L$ is not assumed to be symmetric except briefly at the end of Section 4D.

4A. Some preliminaries on group actions. The following general notation will be used in Section 4A:

- $G$ is a finite flat group scheme over $S$ (not necessarily étale), and

- $\alpha: X \rightarrow S$ is an $S$-scheme of finite type equipped with a strictly free $G$-action (i.e., $G \times{ }_{S} X \rightarrow X \times{ }_{S} X$ via $(g, x) \mapsto(g x, x)$ is a closed immersion) such that every orbit is contained in an affine open set.

Then a general theorem of Grothendieck (cf. [Tate 1997, Theorem 3.4]) ensures that the quotient $Y:=X / G$, along with $\beta: Y \rightarrow S$ and $\xi: X \rightarrow Y$, exists in the category of $S$-schemes. (This is a universal geometric quotient and an fppf quotient. See [van der Geer and Moonen $\geq 2012$, Theorem 4.16, Theorem 4.35] for details.)

Proposition 4.1. Let $\mathscr{F}^{\prime}$ and $\mathscr{F}_{F}$ be a coherent $\mathrm{O}_{X}$-module and a coherent $\mathrm{O}_{Y}$-module, respectively. The canonical maps $\mathscr{F} \rightarrow\left(\xi_{*} \xi^{*} \mathscr{F}^{G}\right)^{G}$ and $\xi^{*}\left(\xi_{*}\left(\mathscr{F}^{\prime}\right)^{G}\right) \rightarrow \mathscr{F}^{\prime}$ are isomorphisms. (The maps are given by the fact that $\xi^{*}$ is the left adjoint of $\xi_{*}$.)

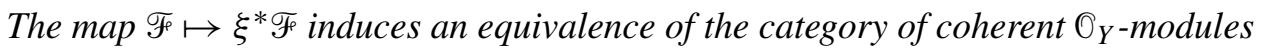
(resp. locally free $\mathrm{O}_{Y}$-modules of finite rank) with the category of coherent $\mathrm{O}_{X}$ modules with $G$-action (resp. locally free $O_{Y}$-modules of finite rank with $G$-action).

Proof. The statement and proof of [Mumford 1974, §12 Theorem 1] can be adapted to the relative setting over $S$. 
Lemma 4.2. The category of $G$-representations on $\mathrm{O}_{S}$-modules has enough injectives.

Proof. Let $\mathscr{F}_{F}$ be a $G$-representation on an $\mathscr{O}_{S}$-module. Since the category of $\mathrm{O}_{S}$-modules has enough injectives, there exists an injective $\hat{O}_{S}$-module $\mathscr{I}$ with $i: \mathscr{F} \hookrightarrow \mathscr{I}$. The $\mathscr{O}_{S}$-module $\widetilde{\mathscr{I}}:=\underline{\operatorname{Hom}}_{\mathbb{O}_{S}}(G, \mathscr{I})$ is an injective object in the category of $G$-representations on $\mathscr{O}_{S}$-modules since, by the injectivity of $\mathscr{I}$, the functor

$$
\mathcal{M} \mapsto \underline{\operatorname{Hom}}_{G}\left(\mathcal{M}, \underline{\operatorname{Hom}}_{\mathbb{O}_{S}}(G, \mathscr{I})\right) \simeq \underline{\operatorname{Hom}}_{\mathbb{O}_{S}}(\mathcal{M}, \mathscr{I})
$$

is exact. (The latter isomorphism is given by $\phi \mapsto(m \mapsto \phi(m)(e))$, where $e$ is the identity of $G$.) The isomorphism for $\mathcal{M}=\mathscr{F}$ yields $\underline{\operatorname{Hom}}_{G}(\mathscr{F}, \widetilde{\mathscr{F}}) \simeq \underline{\operatorname{Hom}}_{\mathscr{O}_{S}}(\mathscr{F}, \mathscr{I})$, and the map $\tilde{i}: \mathscr{F} \rightarrow \tilde{\mathscr{I}}$ corresponding to $i$ is an injection.

There is a functor $\mathscr{V} \mapsto \mathscr{V}^{G}$ from the category of $G$-representations on ${ }^{O_{S}}$ modules to the category of $\mathcal{O}_{S}$-modules. (By [Moret-Bailly 1985, V.1.2], $\mathscr{V}^{G}$ is an $\mathcal{O}_{S}$-module.) For $i \geq 0$, let $\mathscr{H}^{i}(G, \mathscr{V})$ denote the $i$ th right derived functor of the left exact functor $\mathscr{V} \mapsto \mathscr{V}^{G}$.

Lemma 4.3. There is a spectral sequence $E_{2}^{i, j}=\mathscr{H}^{i}\left(G, R^{j} \alpha_{*}\left(\xi^{*} \mathscr{F}\right)\right) \Rightarrow R^{i+j} \beta_{*} \mathscr{F}$. Proof. Let $\operatorname{Rep}(G)$ denote the category of $G$-representations on $O_{S}$-modules. Consider the left exact functors $\operatorname{QCoh}_{Y} \rightarrow \operatorname{Rep}(G)$ and $\operatorname{Rep}(G) \rightarrow \mathrm{QCoh}_{S}$ given by $\mathscr{F} \mapsto \alpha_{*}\left(\xi^{*} \mathscr{F}\right)$ and $\mathscr{V} \mapsto \mathscr{V}^{G}$, respectively. Note that

$$
\alpha_{*}\left(\xi^{*} \mathscr{F}^{G}\right)^{G}=\beta_{*} \mathscr{F} .
$$

The desired spectral sequence is none other than the Grothendieck spectral sequence. We only need to show that the functor $\mathscr{F}_{F} \mapsto \alpha_{*}\left(\xi^{*} \mathscr{F}_{F}\right)$ carries injective objects to acyclic objects.

Set $G_{Y}:=G \times{ }_{S} Y$. Note that $\alpha_{*}\left(\xi^{*} \mathscr{F}\right)=\beta_{*} \xi_{*} \xi^{*} \mathscr{F}_{F}, \xi_{*} \xi^{*} \mathscr{F} \simeq \underline{\operatorname{Hom}}_{\mathbb{O}_{S}}(G, \mathscr{F})$ and

$$
\beta_{*} \underline{\operatorname{Hom}}_{\mathbb{O}_{S}}(G, \mathscr{F}) \simeq \underline{\operatorname{Hom}}_{\mathbb{O}_{S}}\left(G, \beta_{*} \mathscr{F}\right) .
$$

For any $\mathscr{O}_{S}$-module $\mathscr{F}^{\prime}, \underline{\operatorname{Hom}}_{\mathscr{O}_{S}}\left(G, \mathscr{F}^{\prime}\right)$ is acyclic for taking $G$-invariants, so the proof is complete. (The argument is the same as the one showing the acyclicity of induced modules in group cohomology. Indeed, if $\mathscr{F}^{\prime} \rightarrow \mathscr{I}_{\bullet}$ is an injective resolution in $\mathscr{O}_{S}$-modules, then $\underline{\operatorname{Hom}}_{\mathscr{O}_{S}}\left(G, \mathscr{F}^{\prime}\right) \rightarrow \underline{\operatorname{Hom}}_{\mathscr{O}_{S}}\left(G, \mathscr{F}_{\bullet}\right)$ is an injective resolution in $\operatorname{Rep}(G)$. When $G$-invariants are taken, the latter resolution becomes $\mathscr{F}^{\prime} \rightarrow I_{\bullet}$, which is exact.)

Corollary 4.4. Suppose that $R^{j} \alpha_{*}\left(\xi^{*} \mathscr{F}\right)=R^{j} \beta_{*} \mathscr{F}=0$ for an integer $q \geq 0$ unless $j=q$. Then the canonical morphism $R^{q} \beta_{*} \mathscr{F} \rightarrow\left(R^{q} \alpha_{*}\left(\xi^{*} \mathscr{F}\right)\right)^{G}(c f$. Equation (4-1)) is an isomorphism.

Proof. The spectral sequence of Lemma 4.3 degenerates at $E_{2}$ by the assumption and induces the desired isomorphism. 
4B. Construction of adelic Heisenberg representations. Temporarily, we make an assumption that $L$ is nondegenerate of index $i$ for some $i \geq 0$. (This will be removed at the end of this subsection.) Set

$$
\mathscr{q}^{(n)}(L):=R^{i} f_{*}\left(n^{*} L\right) .
$$

Note that $\widetilde{\mathscr{G}}\left(n^{*} L\right)$ acts on $\mathscr{V}^{(n)}(L)$ through its projection onto $\mathscr{G}\left(n^{*} L\right)$, whose action was discussed in Section 2C. For $m, n \in \mathbb{Z}_{>0}$, there is a natural map functorial in $L$

$$
f_{*} n^{*} L \rightarrow f_{*} m_{*} m^{*}\left(n^{*} L\right) \simeq f_{*}(m n)^{*} L
$$

induced by the adjunction map $n^{*} L \rightarrow m_{*} m^{*}\left(n^{*} L\right)$. It works similarly with higher direct image of $f$ (since $m_{*}$ is exact). Let $v_{n, m n}: \mathscr{V}^{(n)}(L) \rightarrow \mathscr{V}^{(m n)}(L)$ be the functorial map $R^{i} f_{*}\left(n^{*} L\right) \rightarrow R^{i} f_{*}\left((m n)^{*} L\right)$. Clearly $v_{m n, m n k} v_{n, m n}=v_{n, m n k}$ for any $m, n, k \in \mathbb{Z}_{>0}$ as both sides are the functorial map with respect to $(m n k)^{*} L \rightarrow n^{*} L$ covering $m k: A \rightarrow A$. Also, $v_{n, m n}$ is compatible with $i_{n, m n}: \widetilde{\mathscr{G}}\left(n^{*} L\right) \hookrightarrow \widetilde{\mathscr{G}}\left((m n)^{*} L\right)$; namely, for all $\gamma \in \widetilde{\mathscr{G}}\left(n^{*} L\right)$ and $v \in \mathscr{V}^{(n)}(L)$, we have $i_{n, m n}(\gamma) \cdot v_{n, m n}(v)=v_{n, m n}(\gamma \cdot v)$. Indeed, this results from the commutativity of

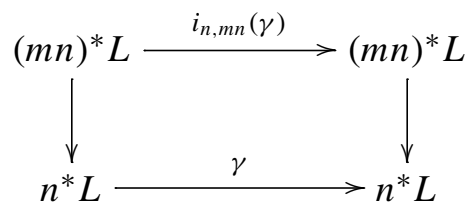

where $\gamma$ and $i_{n, m n}(\gamma)$ act through their respective images in $\mathscr{G}\left(n^{*} L\right)$ and $\mathscr{G}\left((m n)^{*} L\right)$.

Lemma 4.5. As $\mathbb{O}_{S}$-modules, for all $m, n \geq 1$,

$$
\mathscr{V}^{(n)}(L) \simeq\left(\mathscr{V}^{(m n)}(L)\right)^{A[m]}=\left(\mathscr{V}^{(m n)}(L)\right)^{\widetilde{\sigma}_{m n}(n T A)},
$$

where the first isomorphism is induced by $v_{n, m n}$.

Proof. Take $\alpha=\beta=f, \xi=m, \mathscr{F}=n^{*} L$ and $q=i$ in Corollary 4.4 to obtain the first isomorphism

$$
\mathscr{V}^{(n)}(L) \simeq\left(\mathscr{V}^{(m n)}(L)\right)^{A[m]} .
$$

We claim that

$$
\left(\mathscr{V}^{(m n)}(L)\right)^{A[m]}=\left(\mathscr{V}^{(m n)}(L)\right)^{\widetilde{\sigma}_{m n}(n T A)} .
$$

On the right-hand side, $\widetilde{\mathscr{G}}\left((m n)^{*} L\right)$ acts through $\mathscr{G}\left((m n)^{*} L\right)$. The action of the subgroup scheme $\widetilde{\sigma}_{m n}(n T A)$ of $\widetilde{\mathscr{G}}\left((m n)^{*} L\right)$ factors through

$$
\widetilde{\sigma}_{m n}(n T A) / \widetilde{\sigma}_{m n}(m n T A) \simeq A[m] .
$$

In view of Corollary 3.11, the latter $A[m]$-action (on the right-hand side of (4-3)) is described as follows: $x^{\prime} \in A[m]$ acts on $(m n)^{*} L$ via $\psi^{\prime}$, which is obtained from Lemma 3.9 by taking $x=0$ and $\psi=\mathrm{id}$, and this induces the action of $x^{\prime}$ on $\mathscr{V}^{(m n)}(L)$. 
The claim (4-3) follows from the fact that this $A[m]$-action is the same as the one used in Corollary 4.4 and thus used in (4-2). (See Remark 3.10.)

Using the fact that $v_{n, m n}$ are compatible with $i_{n, m n}$ as explained above, we obtain

$$
\widehat{\mathscr{V}}(L):=\underset{n}{\lim } \mathscr{V}^{(n)}(L)
$$

as a $\widehat{\mathscr{G}}(L)$-representation, where $v_{n, m n}$ are transition maps. The $\mathrm{O}_{S}$-module $\widehat{\mathscr{V}}(L)$ carries a weight-1 action by $\widehat{\mathscr{G}}(L)$. Its properties will be investigated in Section 4C.

Remark 4.6. A more concise definition of $\widehat{\mathscr{V}}(L)$ would be $R^{i} f_{*}\left(u^{*} L\right)$. (We defined $u$ in Section 3B.) It is useful to view $\widehat{\mathscr{G}}(L)$ as a compatible system of $\widetilde{\mathscr{G}}\left(n^{*} L\right)$-actions on $\mathscr{V}^{(n)}(L)$ as this allows us to derive properties of $\widehat{\mathscr{V}}(L)$ from those of $\mathscr{V}^{(n)}(L)$.

Now we drop the assumption that the index of $L$ is constant over $S$. Let $g$ be the relative dimension of $A$ over $S$. Since the index function $s \mapsto L_{s}$ is locally constant, we can decompose $S=\coprod_{i=0}^{g} S_{i}$ into open and closed subschemes such that the index function is constantly $i$ on each $S_{i}$. The previous paragraphs construct $q^{(n)}(L), \widehat{\mathscr{V}}(L)$ and so on over each $S_{i}$; thereby, we obtain them over $S$.

4C. Basic properties. Just like at the end of the last subsection, we no longer assume that $L$ has fixed index over $S$.

Definition 4.7. A $\widehat{\mathscr{G}}(L)$-representation $\mathscr{F}$ on an $\widehat{O}_{S}$-module is admissible if $\mathscr{F} \hat{\sigma}(n \cdot T A)$ is a coherent $\hat{O}_{S}$-module for every $n \geq 1$. (We always have that $\mathscr{F}^{\hat{\sigma}(n T A)}$ is an $\mathrm{O}_{S}$-submodule of $\mathscr{F}_{\text {. }}$ )

Definition 4.8. A $\widehat{\mathscr{G}}(L)$-representation $\mathscr{F}$ is smooth if

$$
\mathscr{F}=\bigcup_{n \geq 1} \mathscr{F}^{\hat{\sigma}(n \cdot T A)} .
$$

A useful observation is that $\mathscr{F}^{\hat{\sigma}}(n \cdot T A)$ is a module over $N_{\widehat{g}}(\hat{\sigma}(n T A)) / \hat{\sigma}(n T A) \simeq$ $\mathscr{G}\left(n^{*} L\right)$, cf. Lemma 3.15. This will be exploited several times.

Remark 4.9. If $L$ is a symmetric line bundle so that $\hat{\tau}$ is available, an equivalent criterion for smoothness is that $\mathscr{F}=\bigcup_{n \geq 1} \mathscr{F}^{\hat{\tau}(n \cdot T A)}$. The obvious reason is that $\hat{\sigma}=\hat{\tau}$ on $2 T A$.

Remark 4.10. It is not inconceivable that any weight-1 $\widehat{G}(L)$-representation is smooth, but we have not checked this.

Lemma 4.11. Suppose that $L$ is nondegenerate of index $i$. Then

(i) $\widehat{\mathscr{V}}(L)^{\hat{\sigma}(n T A)}=\mathscr{V}^{(n)}(L)$, and

(ii) the $\widehat{\mathscr{G}}(L)$-representation $\widehat{\mathcal{V}}(L)$ is admissible and smooth. 
Proof. Clearly (i) implies (ii). Part (i) is obtained from Lemma 4.5 by taking limit over $m$. Note that this works even if ind $(L)$ is not constant on $S$, cf. the end of Section 4B.

We give a tentative definition of a (finite) adelic Heisenberg representation. Perhaps a more satisfactory definition is (ii) of Proposition 4.19 below.

Definition 4.12. An adelic Heisenberg representation $\mathscr{H}$ of $\widehat{\mathscr{G}}(L)$ is a smooth $\widehat{\mathscr{G}}(L)$ representation of weight 1 such that $\mathscr{H}^{\hat{\sigma}(n T A)}$ is a locally free $\hat{O}_{S}$-module of rank $n^{2 g} \cdot \operatorname{deg} L$ for all $n \geq 1$.

Lemma 4.13. An adelic Heisenberg representation $\mathcal{H}$ of $\widehat{\mathscr{G}}(L)$ is a locally free $\mathrm{O}_{S}$-module ${ }^{3}$ and irreducible. (The notion of irreducibility is as in Definition 2.11.)

Proof. Let $\mathscr{H}^{\prime} \subset \mathscr{H}$ be a smooth $\widehat{\mathscr{G}}(L)$-subrepresentation, so $\left(\mathscr{H}^{\prime}\right)^{\hat{\sigma}(n T A)} \subset \mathscr{H}^{\hat{\sigma}(n T A)}$ is a $\widetilde{\mathscr{G}}\left(n^{*} L\right)$ - and $\mathscr{G}\left(n^{*} L\right)$-subrepresentation. By Proposition 2.12(i), there is an ideal sheaf $I_{n}$ of $\mathcal{O}_{S}$ such that

$$
\left(\mathscr{H}^{\prime}\right)^{\hat{\sigma}(n T A)}=\mathscr{I}_{n} \cdot \mathscr{H}^{\hat{\sigma}(n T A)} .
$$

By taking $\hat{\sigma}(T A)$-invariants, where $\hat{\sigma}(T A)$ acts through its image in $\widetilde{\mathscr{G}}\left(n^{*} L\right)$, we get

$$
\left(\mathscr{H}^{\prime}\right)^{\hat{\sigma}(T A)}=\mathscr{I}_{n} \cdot \mathscr{H}^{\hat{\sigma}(T A)} .
$$

Since $\mathscr{H}^{\hat{\sigma}(T A)}$ is locally free, the comparison with (4-4) for $n=1$ shows that $\Phi_{n}=\Phi_{1}$ for all $n \geq 1$. Hence, $\mathscr{H}^{\prime}=\mathscr{H} \otimes \mathscr{I}_{1}$. The local freeness of $\mathscr{H}$ follows from the fact that

$$
\mathscr{H}_{s}=\lim _{\longrightarrow}\left(\mathscr{H}^{\hat{\sigma}(n ! T A)}\right)_{s}
$$

is free over $\mathbb{O}_{S, s}$, as it is an increasing union of finite free modules. (Since each transition map has a section, a basis can be written down easily.)

Corollary 4.14. The $\widehat{G}(L)$-representation $\widehat{\mathscr{V}}(L)$ is an adelic Heisenberg representation of $\widehat{\mathscr{G}}(L)$ in the sense of Definition 4.12.

Proof. This follows from Proposition 2.13(ii) and Lemmas 4.11 and 4.13.

Theorem 4.15. Let $\mathcal{H}$ be a Heisenberg representation of $\widehat{\mathscr{G}}(L)$. Then there is an equivalence of categories

$$
\operatorname{Rep}_{\mathrm{sm}}^{1}(\widehat{\mathscr{G}}(L)) \stackrel{\sim}{\rightarrow} \mathrm{QCoh}_{S}
$$

given by $\mathcal{M} \mapsto \underline{\operatorname{Hom}}_{\widehat{G}(L)}(\mathcal{H}, \mathcal{M})$ and $\mathcal{N} \mapsto \mathscr{H} \otimes \mathcal{N}$, which are quasi-inverses of each other.

\footnotetext{
${ }^{3}$ Note that we are dealing with an ${ }^{O} S$-module, which is typically of infinite rank. We consider an $\mathrm{O}_{S}$ module $\mathscr{H}$ locally free if the Zariski localization $\mathscr{H}_{S}$ is a free $\mathscr{O}_{S, s}$-module for all $s \in S$. This does not automatically imply that $\left.\mathscr{H}\right|_{U}$ is a free $\mathcal{O}_{U}$-module in some open neighborhood $U$ of $s$ for a given $s \in S$.
} 
Proof. To simplify notation, let us write $\widehat{\mathscr{V}}$ for $\widehat{\mathscr{V}}(L)$. Suppose that the proposition is known for $\mathscr{H}=\widehat{\mathscr{V}}$. Then it implies that any Heisenberg representation $\mathscr{H}^{\prime}$ is isomorphic to $\widehat{\mathscr{V}} \otimes \mathscr{F}$ for an $\widehat{O}_{S}$-module $\mathscr{F}$. Since the $\hat{\sigma}(T A)$-invariants in $\mathscr{H}^{\prime}$ and $\widehat{\mathscr{V}}$ are locally free of the same rank, we see that $\mathscr{F}_{F}$ is an invertible $\hat{O}_{S}$-module. By using this, the proposition for $\mathscr{H}^{\prime}$ is easily deduced from the case for $\widehat{\mathscr{V}}$.

Consider the case $\mathscr{H}=\widehat{\mathscr{V}}$. We will show that the natural map

$$
\widehat{\mathscr{V}} \otimes \underline{\operatorname{Hom}}_{\widehat{G}}(L)(\widehat{\mathscr{V}}, M) \rightarrow M
$$

sending $v \otimes f$ to $f(v)$, which is clearly functorial in $\mathcal{M}$, is an isomorphism in $\operatorname{Rep}_{\mathrm{sm}}^{1}(\widehat{\mathscr{G}}(L))$. Once this is shown, the same argument as on page 113 of [MoretBailly 1985] proves that $\mathcal{N} \rightarrow \underline{\operatorname{Hom}}_{\widehat{G}(L)}(\widehat{\mathscr{V}}, \widehat{\mathscr{V}} \otimes \mathcal{N})$ is a functorial isomorphism in $\mathrm{QCoh}_{S}$, and we will be done. As a preparation, let us consider the functors

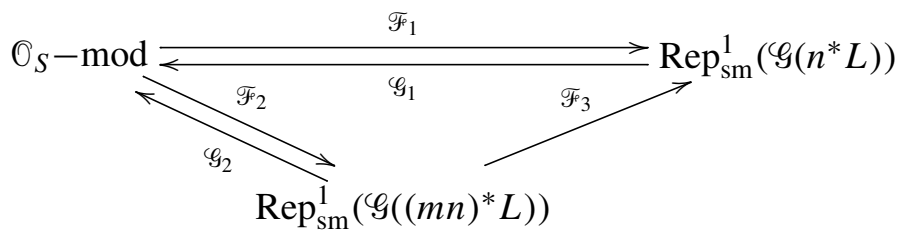

where $\mathscr{F}_{1}, \mathscr{F}_{2}, \mathscr{G}_{1}$ and $\mathscr{G}_{2}$ are the functors in Proposition 2.12(ii), which give equivalences of categories, and $\mathscr{F}_{3}$ is given by the rule $\mathscr{F}_{3}\left(\mu_{0}\right)=\mathcal{M}_{0}^{A[m]}$. Then $\mathscr{F}_{3} \circ \mathscr{F}_{2} \simeq \mathscr{F}_{1}$ canonically. Indeed, in view of Lemma 4.11(i),

$$
\mathscr{F}_{1}\left(M_{0}\right)=M_{0} \otimes \mathscr{V}^{(n)} \simeq\left(M_{0} \otimes \mathscr{V}^{(m n)}\right)^{A[m]}=\mathscr{F}_{3}\left(\mathscr{F}_{2}\left(M_{0}\right)\right) .
$$

Now let $\mathcal{M} \in \operatorname{Rep}_{\mathrm{sm}}^{1}(\widehat{G}(L))$, and set $\mathcal{M}^{(n)}:=\mathcal{M}^{\hat{\sigma}(n T A)}$ for $n \geq 1$. It is implied by $\mathscr{F}_{3} \circ \mathscr{F}_{2} \simeq \mathscr{F}_{1}$ that canonically

$$
\mathscr{G}_{1} \mathscr{F}_{3}\left(\mathscr{F}_{2} \mathscr{G}_{2}\right) \simeq\left(\mathscr{G}_{1} \mathscr{F}_{1}\right) \mathscr{G}_{2}
$$

Thanks to Proposition 2.12(ii), we get a canonical isomorphism $\mathscr{G}_{1} \mathscr{F}_{3} \simeq \mathscr{G}_{2}$. Applying to $\mathcal{M}^{(m n)}$ and unraveling the functors, we have a canonical isomorphism

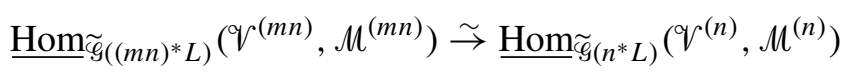

induced by the restriction to $\mathscr{V}^{(n)}$. (The right-hand side of (4-6) is a rewriting of $\underline{\operatorname{Hom}}_{\mathscr{G}\left(n^{*} L\right)}\left(\mathscr{V}^{(n)}, \mathcal{M}^{(n)}\right)$, where we use the fact that $\hat{\sigma}(n T A)$ is trivial on $\mathscr{V}^{(n)}$ and $\mathcal{M}^{(n)}$. What happens to the left-hand side is similar.) We obtain the following commutative diagram in which the vertical maps come from natural inclusions 
$\mathscr{V}^{(n)} \hookrightarrow \mathscr{V}^{(m n)}, \mathcal{M}^{(n)} \hookrightarrow \mathcal{M}^{(m n)}$ and (4-6):

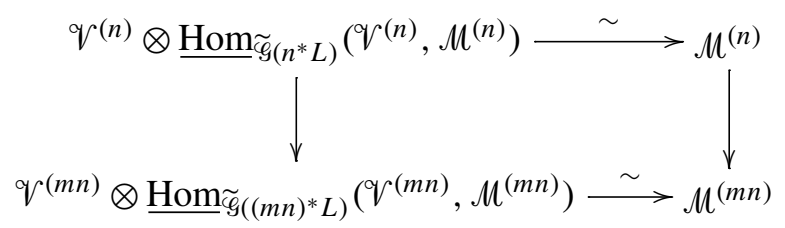

By taking limit over $n$, we deduce that (4-5) is an isomorphism.

Corollary 4.16. The canonical map $\mathrm{O}_{S} \rightarrow \underline{\operatorname{End}}_{\widehat{\varphi}_{(L)}}(\mathscr{H})$ (via the $\mathrm{O}_{S}$-module structure on $\mathscr{H})$ is an isomorphism.

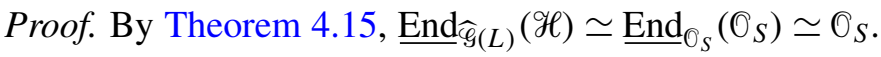

Corollary 4.17. Let $\mathcal{H}$ be as in Lemma 4.13. If $\mathscr{H}^{\prime}$ is another $\widehat{\mathscr{G}}(L)$-representation with the same property, then there exists an invertible $\mathbb{O}_{S}$-module $\mathcal{N}$ such that

$$
\mathscr{H}^{\prime} \simeq \mathscr{H} \otimes \mathcal{N} .
$$

Proof. This is proved as in the first paragraph of the proof of Theorem 4.15.

Corollary 4.18. Suppose that $S=\operatorname{Spec} R$ for a local ring $R$. Then any two Heisenberg representations are isomorphic.

Proof. Immediate from Corollary 4.17.

This subsection ends with an alternative characterization of Heisenberg representations. It will be used in Section 5A.

Proposition 4.19. The following are equivalent:

(i) $\mathcal{H}$ is a Heisenberg representation of $\widehat{\mathscr{G}}(L)$. (See Definition 4.12.)

(ii) $\mathcal{H}$ is a weight-1 admissible smooth irreducible representation of $\widehat{\mathscr{G}}(L)$ on a locally free $\mathrm{O}_{S}$-module such that $\mathcal{H}$ does not vanish anywhere on $S$.

Remark 4.20. In (ii) above, it is enough to require $\mathscr{H} \neq 0$ when $S$ is connected. On the other hand, one could show that the admissibility in (ii) is superfluous by extending Lemma 4.22 to the case when $\mathscr{F}$ may be of infinite rank. That proof is easily reduced to the finite rank situation.

Proof. Lemma 4.13 says that (i) implies (ii). In order to show the other implication, let $\mathscr{H}$ be as in (ii) and $\mathscr{H}^{\prime}$ be a Heisenberg representation of $\widehat{\mathscr{G}}(L)$ (in the sense of Definition 4.12). Theorem 4.15 tells us that $\mathscr{H} \simeq \mathscr{H}^{\prime} \otimes \mathcal{N}$ for some $\mathcal{O}_{S}$-module $\mathcal{N}$. By taking invariants under $\hat{\sigma}(n T A)$ for a large enough $n$ (so that the invariants are nontrivial), we see that $\mathcal{N}$ has to be a coherent $\mathcal{O}_{S}$-module. It suffices to show that $\mathcal{N}$ is locally free of rank 1 .

Choose an arbitrary $s \in S$. The stalks at $s$ are related by $\mathscr{H}_{s} \simeq \mathscr{H}_{s}^{\prime} \otimes_{\mathcal{O}_{S, s}} \mathcal{N}_{s}$. We see that $\mathcal{N}_{s}$ is a projective $\mathcal{O}_{S, s}$-module as $\mathscr{H}_{s}^{\prime}$ and $\mathscr{H}_{s}$ are free over $\mathcal{O}_{S, s}$. Since $\mathcal{N}_{s}$ is 
finitely generated over the noetherian ring $\mathrm{O}_{S, s}$, it is free of finite rank. Now let $U$ be an open affine noetherian neighborhood of $s$ in $S$. The proof will be complete if $\left.\mathcal{N}\right|_{U}$ is shown to be an invertible $\mathcal{O}_{U}$-module.

Suppose this is not the case. Lemma 4.23 tells us that $\left.\mathcal{N}\right|_{U}$ has an $\mathfrak{O}_{U}$-submodule $\mathcal{M}$ that is not given as $\left.\mathcal{N}\right|_{U} \otimes_{\mathcal{O}_{U}} \mathscr{F}$ for any ideal sheaf $\mathscr{F} \subset \mathcal{O}_{U}$. Applying Lemma 4.22, we obtain an $\hat{O}_{S}$-submodule $\mathcal{N}^{\prime}$ of $\mathcal{N}$ such that $\left.\mathcal{N}^{\prime}\right|_{U}=\mathcal{M}$. Then it is impossible that $\mathcal{N}^{\prime}=\mathcal{N} \otimes_{\mathcal{O}_{S}} \mathscr{I}$ for an ideal sheaf $\mathscr{I} \subset \mathcal{O}_{S}$. (If it were possible, by restricting to $U$, we would get $\mathcal{M}=\left.\mathcal{N}_{U} \otimes_{\mathscr{O}_{U}} \mathscr{I}\right|_{U}$, but this is a contradiction.) This means, via Theorem 4.15 (applicable to $\mathscr{H}^{\prime}$ ), that $\mathscr{H}$ allows a $\widehat{\mathscr{G}}(L)$-subrepresentation $\mathscr{H}^{\prime} \otimes \mathcal{N}^{\prime}$ not given by an ideal sheaf, contradicting the assumption that $\mathcal{H}$ is irreducible.

Corollary 4.21. Consider $\left(A^{\prime}, L^{\prime}\right)$ and $(A, L)$ with a bounded isogeny $\alpha: A^{\prime} \rightarrow A$ such that $L^{\prime}=\alpha^{*} L$. Let $\widehat{\mathscr{G}}(\alpha): \widehat{\mathscr{G}}\left(L^{\prime}\right) \stackrel{\sim}{\rightarrow} \widehat{\mathscr{G}}(L)$ be defined as in Lemma 3.13. If $\rho: \widehat{\mathscr{G}}(L) \rightarrow$ Aut $_{\mathrm{O}_{S}}(\mathscr{H})$ is a Heisenberg representation, then $\rho \circ \widehat{\mathscr{G}}(\alpha)$ is a Heisenberg representation of $\widehat{\mathscr{G}}\left(L^{\prime}\right)$.

Proof. This is clear from criterion (ii) of Proposition 4.19 and Lemma 3.16. (Thanks to the latter, the fact that $\rho$ is admissible and smooth shows that $\rho \circ \widehat{\mathscr{G}}(\alpha)$ is also.)

The following two lemmas were used in the proof of Proposition 4.19:

Lemma 4.22. Let $\mathscr{F}_{F}$ be an $\mathrm{O}_{S}$-module and $U$ an open affine subscheme of $S$. Let $M$ be an $\mathrm{O}_{U}$-module defined by an $\mathrm{O}_{S}(U)$-submodule of $\mathscr{F}(U)$. Define a Zariski presheaf $\mathscr{F}^{\prime}$ on $S$ by

$$
\mathscr{F}^{\prime}(V)=\left\{a \in \mathscr{F}(V)|a|_{U \cap V} \in \mathcal{M}(U \cap V)\right\} .
$$

Then $\mathscr{F}^{\prime}$ is a Zariski sheaf and an $\mathrm{O}_{S}$-submodule of $\mathscr{F}_{\text {. }}$ (Recall that every $\mathrm{O}_{S}$-module (likewise, every $\mathrm{O}_{U}$-module) is required to be quasicoherent in our convention.)

Proof. It is a routine check that $\mathscr{F}^{\prime}$ is a Zariski sheaf. By construction, $\mathscr{F}^{\prime}$ is a subsheaf of $\mathscr{F}$. The verification that $\mathscr{F}^{\prime}$ is an $\mathbb{O}_{S}$-module reduces to the affine case, in which case it is elementary.

Lemma 4.23. Let $U$ be a noetherian scheme. Let $\mathscr{F}_{F}$ be a locally free $\mathrm{O}_{U}$-module of finite rank. Suppose that $\mathscr{F}$ has rank at least 1 at every point of $U$ and rank greater than 1 at some point $u \in U$. (Note that $U$ may not be connected.) Then there exists an $\mathfrak{O}_{U}$-submodule $M \subset \mathscr{F}$ that is not of the form $\mathcal{M}=\mathscr{F}_{\mathbb{F}} \otimes_{U} \mathscr{I}$ for any ideal sheaf $\mathscr{I} \subset \mathrm{O}_{U}$.

Proof. We can find an affine subscheme $V=\operatorname{Spec} B$ of $U$ containing $u$ on which $\left.\mathscr{F}\right|_{V}$ is free of rank at least 2. Let $M$ be any rank-1 free $B$-submodule of $\mathscr{F}(V)$, and denote by $\mathcal{M}^{\prime}$ the corresponding $\mathscr{O}_{V}$-module. Extend $\mathcal{M}^{\prime}$ to an $\mathscr{O}_{U}$-module $\mathcal{M}$ by the previous lemma. We claim that $\mathcal{M}$ satisfies the condition of the lemma. Indeed, if we had $M=\mathscr{F} \otimes_{\mathscr{O}_{U}} \mathscr{I}$ for some ideal $\mathscr{I} \subset \mathcal{O}_{U}$, then we would reach a 
contradiction by taking stalk at $u$ and computing the $k(u)$-dimension after tensoring $k(u):=\mathfrak{O}_{U, u} / m_{U, u}$. (Here $m_{U, u}$ denotes the unique maximal ideal of $\mathfrak{O}_{U, u}$.)

4D. Dual Heisenberg representations and matrix coefficients. As we have seen in Section 2D, there are isomorphisms of $\mathscr{G}\left(n^{*} L\right) \times \mathscr{G}\left(n^{*} L\right)$-representations

$$
\mathscr{V}\left(n^{*} L\right) \otimes \mathscr{V}\left(n^{*} L\right)^{\vee} \stackrel{\sim}{\rightarrow} \underline{\operatorname{Hom}}_{\mathbb{G}_{m}}\left(\mathscr{G}\left(n^{*} L\right), \mathscr{O}_{S}\right)
$$

for varying $n$. On the right-hand side, $\mathbb{G}_{m}$ acts on $\mathscr{G}\left(n^{*} L\right)$ and $\mathcal{O}_{S}$ by multiplication. We will promote (4-7) to an adelic isomorphism.

Definition 4.24. Define an $\mathrm{O}_{S}$-module

$$
\begin{aligned}
\underline{\operatorname{Hom}}_{\mathbb{G}_{m}}^{\mathrm{sm}}\left(\widehat{\mathscr{G}}(L), \widehat{O}_{S}\right): & =\bigcup_{n \geq 1} \underline{\operatorname{Hom}}_{\mathbb{G}_{m}}\left(\widehat{G}(L) / \hat{\sigma}(n T A), O_{S}\right) \\
& =\bigcup_{n \geq 1} \underline{\operatorname{Hom}}_{\mathbb{G}_{m}}\left(\widehat{\mathscr{G}}(L), \widehat{O}_{S}\right)^{\hat{\sigma}(n T A) \times\{1\}} .
\end{aligned}
$$

A section $\phi$ of $\underline{\operatorname{Hom}}_{\mathbb{G}_{m}}\left(\widehat{\mathscr{G}}(L), \mathscr{O}_{S}\right)$ is said to be smooth if it is a section of the above $\mathrm{O}_{S}$-module. (The definition is equivalent if $\hat{\tau}$ is used in place of $\hat{\sigma}$; cf. Remark 4.9.)

Lemma 4.25. The map

$$
\begin{gathered}
\widehat{\mathscr{V}}(L) \otimes \widehat{\mathscr{V}}(L)^{\vee} \rightarrow \underline{\operatorname{Hom}}_{\mathbb{G}_{m}}^{\mathrm{sm}}\left(\widehat{\mathscr{G}}(L), O_{S}\right) \\
v \otimes v^{\vee} \mapsto\left(\gamma \mapsto v^{\vee}(\gamma v)\right)
\end{gathered}
$$

is an isomorphism of $\widehat{\mathscr{G}}(L) \times \widehat{\mathscr{G}}(L)$-representations. Here, $\widehat{\mathscr{V}}(L)^{\vee}$ is equipped with an action of $\widehat{G}(L)$ by the same formula as (2-2). On the right-hand side, the action is described by $\left(\left(\gamma_{1}, \gamma_{2}\right) \psi\right)(\gamma)=\psi\left(\gamma_{2}^{-1} \gamma \gamma_{1}\right)$ for $\psi \in \underline{\operatorname{Hom}}_{\mathbb{G}_{m}}^{\mathrm{sm}}\left(\widehat{\mathscr{G}}(L), \mathscr{O}_{S}\right)$ and $\gamma_{1}, \gamma_{2} \in \widehat{\mathscr{G}}(L)$.

Proof. Recall from Lemma 3.15 that $\widetilde{G}\left(n^{*} L\right) / \hat{\sigma}(n T A) \simeq \mathscr{G}\left(n^{*} L\right)$ naturally. Thus, (4-7) may be rewritten as

$$
\begin{aligned}
\mathscr{V}\left(n^{*} L\right) \otimes \mathscr{V}\left(n^{*} L\right)^{\vee} \stackrel{\sim}{\rightarrow} \underline{\operatorname{Hom}}_{\mathbb{G}_{m}}\left(\widetilde{\mathscr{G}}\left(n^{*} L\right), \mathcal{O}_{S}\right)^{\hat{\sigma}(n T A) \times \hat{\sigma}(n T A)} \\
=\underline{\operatorname{Hom}}_{\mathbb{G}_{m}}\left(\widetilde{\mathscr{G}}\left(n^{*} L\right), \mathcal{O}_{S}\right)^{\hat{\sigma}(n T A) \times\{1\}},
\end{aligned}
$$

where the last equality holds thanks to Lemma 3.15(ii). By taking further invariant, we obtain

$$
\mathscr{V}(L) \otimes \mathscr{V}\left(n^{*} L\right)^{\vee} \stackrel{\sim}{\rightarrow} \underline{\operatorname{Hom}}_{\mathbb{G}_{m}}\left(\widetilde{\mathscr{G}}\left(n^{*} L\right), \mathscr{O}_{S}\right)^{\hat{\sigma}(T A) \times\{1\}}
$$

as maps of $\widetilde{G}(L) \times \widetilde{\mathscr{G}}\left(n^{*} L\right)$-representations. (Note that $\mathscr{V}(L)$ is acted upon by $\left.N_{\widetilde{G}\left(n^{*} L\right)}(\hat{\sigma}(T A)) / \hat{\sigma}(T A)=\widetilde{\mathscr{G}}(L) / \hat{\sigma}(T A).\right)$

We patch these isomorphisms via inverse limit, which are compatible as $n$ varies (as they are given by the same formula as (4-9)), to obtain an isomorphism of 
$\widetilde{\mathscr{G}}(L) \times \widehat{\mathscr{G}}(L)$-representations

$$
\mathscr{V}(L) \otimes \widehat{\mathscr{V}}(L)^{\vee} \stackrel{\sim}{\rightarrow} \underline{\operatorname{Hom}}_{\mathbb{G}_{m}}\left(\widehat{\mathscr{G}}(L), \mathscr{O}_{S}\right)^{\hat{\sigma}(T A) \times\{1\}} .
$$

Likewise, there is an isomorphism of $\widetilde{\mathscr{G}}\left(n^{*} L\right) \times \widehat{\mathscr{G}}(L)$-representations

$$
\mathscr{V}\left(n^{*} L\right) \otimes \widehat{\mathscr{V}}(L)^{\vee} \stackrel{\sim}{\rightarrow} \underline{\operatorname{Hom}}_{\mathbb{G}_{m}}\left(\widehat{\mathscr{G}}(L), O_{S}\right)^{\hat{\sigma}(n T A) \times\{1\}}
$$

given by the same formula as (4-9). By patching again, we arrive at the map (4-8) and see that it is an isomorphism.

Corollary 4.26. For any Heisenberg representation He, (4-9) induces an isomorphism of $\widehat{\mathscr{G}}(L) \times \widehat{\mathscr{G}}(L)$-representations

$$
\mathscr{H} \otimes \mathscr{H}^{\vee} \simeq \underline{\operatorname{Hom}}_{\mathbb{G}_{m}}^{\mathrm{sm}}\left(\widehat{\mathscr{G}}(L), \mathrm{O}_{S}\right)
$$

Proof. Corollary 4.17 tells us that $\mathscr{H} \otimes \mathscr{H}^{\vee} \simeq \widehat{\mathscr{V}}(L) \otimes \widehat{V}(L)^{\vee}$ canonically. Composing this with (4-8), we derive the desired isomorphism.

Definition 4.27. Set $\underline{\operatorname{Hom}}_{O_{S}}^{\mathrm{sm}}\left(V A, O_{S}\right):=\bigcup_{n \geq 1} \underline{\operatorname{Hom}}_{{ }_{S}}\left(V A / n T A, \mathscr{O}_{S}\right)$. A section of $\underline{\operatorname{Hom}}_{O_{S}}^{\mathrm{sm}}\left(V A, \mathrm{O}_{S}\right)$ is said to be smooth.

From here until the end of this subsection, assume in addition that $L$ is symmetric. There is a further isomorphism of $\mathrm{O}_{S}$-modules

$$
\underline{\operatorname{Hom}}_{\mathbb{G}_{m}}\left(\widehat{G}(L), O_{S}\right) \simeq \underline{\operatorname{Hom}}_{\mathbb{O}_{S}}\left(V A, O_{S}\right)
$$

by restricting from $\widehat{\mathscr{G}}(L) \simeq \mathbb{G}_{m} \times V A$ (Lemma 3.18) to $\{1\} \times V A$. Then (4-11) induces an isomorphism from $\underline{\operatorname{Hom}}_{\mathbb{G}_{m}}^{\mathrm{sm}}\left(\widehat{\mathscr{G}}(L), \mathscr{O}_{S}\right)$ onto $\underline{\operatorname{Hom}}^{\mathrm{sm}}\left(V A, \mathcal{O}_{S}\right)$, and the $\widehat{\mathscr{G}}(L) \times \widehat{\mathscr{G}}(L)$-action may be transported to them. This action will be used in the following corollary:

Corollary 4.28. Suppose $\operatorname{deg} L=1$. There is a Heisenberg representation Ho so that

$$
\begin{aligned}
\mathscr{H}^{\vee} & =\underline{\operatorname{Hom}}_{\mathrm{O}_{S}}\left(V A, O_{S}\right)^{\hat{\sigma}(T A) \times\{1\}} \\
& =\left\{\phi \in \underline{\operatorname{Hom}}_{O_{S}}\left(V A, O_{S}\right) \mid \phi(x)=e_{*}^{L}\left(\frac{1}{2} y\right) \hat{e}^{L}\left(\frac{1}{2} x, y\right) \cdot \phi(x+y), \forall x \in V A, y \in T A\right\} \\
& =\left\{\phi \in \underline{\operatorname{Hom}}_{\bigcirc_{S}}^{\mathrm{sm}}\left(V A, O_{S}\right) \mid \phi(x)=e_{*}^{L}\left(\frac{1}{2} y\right) \hat{e}^{L}\left(\frac{1}{2} x, y\right) \cdot \phi(x+y), \forall x \in V A, y \in T A\right\} .
\end{aligned}
$$

The action of $(\lambda, z) \in \mathbb{G}_{m} \times V A \simeq \widehat{\mathscr{G}}(L)(c f$. Lemma 3.18, (3-10)) is described by

$$
((\lambda, z) \phi)(x)=\lambda^{-1} \hat{e}^{L}(x, z / 2) \cdot \phi(x-z) .
$$

Proof. Set $\mathcal{H}:=\widehat{\mathscr{V}}(L) \otimes \mathscr{V}(L)^{\vee}$. By the assumption $\mathscr{V}(L)$ is an invertible $\mathscr{O}_{S}$-module. The isomorphism (4-10) provides

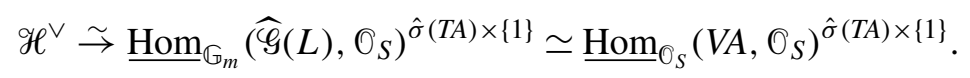


Let $y \in T A$. If $\phi \in \underline{\operatorname{Hom}}_{\mathbb{G}_{m}}\left(\widehat{\mathscr{G}}(L), \mathscr{O}_{S}\right)$, then $\hat{\sigma}(y)=\hat{\tau}(y) e_{*}^{L}(y / 2)$ acts on $\phi$ as follows, where the elements of $\widehat{\mathscr{G}}(L)$ are written using Lemma 3.18:

$$
\begin{aligned}
(\hat{\sigma}(y) \cdot \phi)((1, x)) & =\phi\left(e_{*}^{L}\left(\frac{1}{2} y\right)(1, x)(1, y)\right) \\
& =e_{*}^{L}\left(\frac{1}{2} y\right) \cdot \phi\left(\left(\hat{e}^{L}\left(\frac{1}{2} x, y\right), x+y\right)\right) \\
& =e_{*}^{L}\left(\frac{1}{2} y\right) \hat{e}^{L}\left(\frac{1}{2} x, y\right) \cdot \phi((1, x+y)) .
\end{aligned}
$$

Thus, the condition that $\hat{\sigma}(y) \cdot \phi=\phi$ for all $y \in T A$ produces the transformation formula for $\phi$. Such a $\phi$ is automatically smooth. Indeed, for any $x \in V A$, choose $n \geq 1$ such that $x \in \frac{1}{n} T A$. The transformation formula tells us that $\phi(x+y)=\phi(x)$ for all $y \in 2 n T A$ since $\left.e_{*}^{L}\right|_{2 T A} \equiv 1$ and $\left.\hat{e}^{L}\right|_{T A \times T A} \equiv 1$.

To compute the group action, let $\psi \in \underline{\operatorname{Hom}}_{\mathbb{G}_{m}}\left(\widehat{\mathscr{G}}(L), O_{S}\right)$ be the map corresponding to $\phi$ via (4-11). Then (using Lemma 3.18 in the third equality)

$$
\begin{aligned}
((\lambda, z) \phi)(x) & =(\lambda \hat{\tau}(z) \psi)(\hat{\tau}(x))=\lambda^{-1} \psi\left(\hat{\tau}(z)^{-1} \hat{\tau}(x)\right) \\
& =\lambda^{-1} \psi\left(\hat{e}^{L}(z / 2, x)^{-1} \hat{\tau}(x-z)\right)=\lambda^{-1} \hat{e}^{L}(z / 2, x)^{-1} \psi(\hat{\tau}(x-z)) \\
& =\lambda^{-1} \hat{e}^{L}(x, z / 2) \cdot \phi(x-z) .
\end{aligned}
$$

Remark 4.29. Corollary 4.28 may be thought of as presenting the (dual) lattice model for $\mathscr{H}^{\vee}$, whose dual gives rise to the lattice model for $\mathscr{H}$.

Remark 4.30. Although $\mathscr{H}$ is a smooth $\widehat{\mathscr{G}}(L)$-representation, there is no reason to expect $\mathscr{H}^{\vee}$ to be smooth in general. We caution the reader that the smoothness of $\phi$ in Corollary 4.28 does not imply that $\mathscr{H}^{\vee}$ is smooth as a $\widehat{\mathscr{G}}(L)$-representation.

Remark 4.31. Let us assume that $L$ has index 0 , namely that $L$ is relatively ample. By choosing a particular section $l_{0} \in H^{0}\left(S, \widehat{\mathscr{V}}(L)^{\vee}\right)$, one can associate theta functions for each element of $H^{0}(S, \widehat{V}(L))$ as explained in [Mumford 2007, §5, Application 2]. More precisely, take $l_{0}$ to be "the evaluation at 0 " map $\widehat{\mathscr{V}}(L) \rightarrow \mathcal{O}_{S}$. Then (4-8) (by taking $v^{\vee}=l_{0}$ ) and (4-11) induce a map $H^{0}(S, \widehat{\mathscr{V}}(L)) \rightarrow \operatorname{Hom}_{\mathscr{O}_{S}}\left(V A, \mathcal{O}_{S}\right)$, which is a geometric construction of theta functions.

4E. An application of the Künneth formula, Part I. For $r=1,2$, let $f_{r}: A_{r} \rightarrow S$ be an abelian scheme with a nondegenerate line bundle $L_{r}$ of index $i_{r}$. Define $A:=A_{1} \times{ }_{S} A_{2}$ with projections $p_{r}: A \rightarrow A_{r}$ and the structure map $f: A \rightarrow S$. Take $L:=p_{1}^{*} L_{1} \otimes p_{2}^{*} L_{2}$.

Lemma 4.32. We have canonical isomorphisms

$$
R^{j} f_{*} L= \begin{cases}R^{i_{1}} f_{1, *} L_{1} \otimes R^{i_{2}} f_{2, *} L_{2} & \text { if } j=i_{1}+i_{2}, \\ 0 & \text { if } j \neq i_{1}+i_{2} .\end{cases}
$$

In particular, $L$ is nondegenerate of index $i_{1}+i_{2}$. 
Proof. This is a consequence of [Grothendieck 1963, Théorème 6.7.8]. (Take the two complexes of $\mathrm{O}_{S}$-modules there to be $L_{1}$ and $L_{2}$, where each of them is viewed as a complex concentrated in degree 0. .)

By checking that the isomorphisms in Lemma 4.32 for $n^{*} L$ are compatible with transition maps for varying $n$ (namely $v_{n, m n}$ in Section 3B and its analogues for $\left(A_{1}, L_{1}\right)$ and $\left.\left(A_{2}, L_{2}\right)\right)$, we obtain a canonical isomorphism

$$
\widehat{\mathscr{V}}\left(L_{1}\right) \otimes \widehat{\mathscr{V}}\left(L_{2}\right) \simeq \widehat{\mathscr{V}}(L)
$$

Moreover, we have a natural embedding

$$
\mathscr{G}\left(n^{*} L_{1}\right) \times \mathscr{G}\left(n^{*} L_{2}\right) \hookrightarrow \mathscr{G}\left(n^{*} L\right)
$$

for each $n \geq 1$, sending $\left(\left(\phi_{1}, x_{1}\right),\left(\phi_{2}, x_{2}\right)\right)$ to $\left(p_{1}^{*} \phi_{1} \otimes p_{2}^{*} \phi_{2},\left(x_{1}, x_{2}\right)\right)$. This map lifts to a map $\widetilde{\mathscr{G}}\left(n^{*} L_{1}\right) \times \widetilde{\mathscr{G}}\left(n^{*} L_{2}\right) \hookrightarrow \widetilde{\mathscr{G}}\left(n^{*} L\right)$ and patches to

$$
\widehat{\mathscr{G}}\left(n^{*} L_{1}\right) \times \widehat{\mathscr{G}}\left(n^{*} L_{2}\right) \hookrightarrow \widehat{\mathscr{G}}\left(n^{*} L\right) .
$$

It is a routine check that (4-12) is equivariant with respect to (4-13). Namely, the restriction of the $\widehat{\mathscr{G}}\left(n^{*} L\right)$-representation $\widehat{\mathscr{V}}(L)$ to $\widehat{\mathscr{G}}\left(n^{*} L_{1}\right) \times \widehat{\mathscr{G}}\left(n^{*} L_{2}\right)$ via (4-13) is identified via (4-12) with the $\widehat{\mathscr{G}}\left(n^{*} L_{1}\right) \times \widehat{\mathscr{G}}\left(n^{*} L_{2}\right)$-representation on $\widehat{\mathscr{V}}\left(L_{1}\right) \otimes \widehat{\mathscr{V}}\left(L_{2}\right)$. In Section 5C, we will see an analogous result for Weil representations.

4F. Representations of p-adic Heisenberg groups. We return to the $p$-adic setup of Section 3F; in particular, $\operatorname{deg} L$ is assumed to be a power of a prime $p$. Define a $\widehat{\mathscr{G}}_{p}(L)$-representation

$$
\widehat{\mathscr{V}}_{p}(L):=\underset{n}{\lim } \mathscr{V}^{\left(p^{n}\right)}(L)
$$

The admissibility and smoothness are defined for $\widehat{\mathscr{G}}_{p}(L)$-representations as in Definitions 4.7 and 4.8 by letting $n$ run over powers of $p$. A Heisenberg representation of $\widehat{\mathscr{G}}_{p}(L)$ is defined exactly as in Proposition 4.19(ii) and induces an equivalence of categories as in Theorem 4.15. The representation $\widehat{\mathscr{V}}_{p}(L)$ is a Heisenberg representation of $\widehat{\mathscr{G}}_{p}(L)$, and any two Heisenberg representations differ by a tensoring with a line bundle over $S$. We also have the analogues of results in Section 4D and Section 4E.

\section{Weil representations}

As in the previous section, let $A$ be an abelian scheme over a locally noetherian scheme $S$. Now $L$ is a nondegenerate symmetric line bundle over $A$. 
5A. Adelic Weil representations. Let $\rho: \widehat{\mathscr{G}}(L) \rightarrow{\underline{\text { Aut }}}_{{ }_{S}}(\mathscr{H})$ be any adelic Heisenberg representation (Definition 4.12, cf. Proposition 4.19). Define a group functor $\mathrm{Sp}^{\mathrm{b}}\left(V A, \hat{e}^{L}\right)$ on $(\mathrm{Sch} / S)$ by

$$
\underline{\mathrm{Sp}}^{\mathrm{b}}\left(V A, \hat{e}^{L}\right)(T)=\left\{g \in \underline{\operatorname{Aut}}_{T}^{\mathrm{b}}\left(V A \times_{S} T\right) \mid \hat{e}^{L} \circ(g, g)=\hat{e}^{L}\right\} .
$$

(The superscript b stands for "bounded".) Note that $g \in \underline{S p}^{\mathrm{b}}\left(V A, \hat{e}^{L}\right)(T)$ acts on $\mathbb{G}_{m}(T) \times V A(T)$ by $g \cdot(\lambda, x)=(\lambda, g x)$ and that this action preserves the group law of (3-10). The automorphism $\widehat{\mathscr{G}}(L) \simeq \mathbb{G}_{m} \times V A$ of Lemma 3.18 allows us to transport the $\operatorname{Sp}^{\mathrm{b}}\left(V A, \hat{e}^{L}\right)$-action to the side of $\widehat{\mathscr{G}}(L)$.

Let $T$ be a locally noetherian $S$-scheme. Write $L_{T}:=L \times_{S} T$, and define $\rho_{T}: \widehat{\mathscr{G}}(L) \times T \rightarrow{\text { Aut }_{\widehat{O}_{T}}}\left(\mathcal{H} \otimes \mathcal{O}_{T}\right)$ to be the representation induced from $\rho$ by base extension. It can be seen from the construction of $\widehat{\mathscr{G}}(L)$ that $\widehat{\mathscr{G}}(L) \times T \simeq \widehat{\mathscr{G}}\left(L_{T}\right)$ canonically. Moreover, $\mathscr{H} \otimes \mathcal{O}_{T}$ is a Heisenberg representation of $\widehat{\mathscr{G}}\left(L_{T}\right)$. For each $g \in \underline{\operatorname{Sp}}^{\mathrm{b}}\left(V A, \hat{e}^{L}\right)(T)$, define $\rho_{T}^{g}:=\rho_{T} \circ g$, a weight-1 representation of $\widehat{\mathscr{G}}\left(L_{T}\right)$.

\section{Lemma 5.1.}

(i) $\rho_{T}^{g}$ is a Heisenberg representation of $\widehat{\mathscr{G}}\left(L_{T}\right)$.

(ii) $\rho_{T}^{g} \simeq \rho_{T}$ as $\widehat{\mathscr{G}}\left(L_{T}\right)$-representations.

Proof. Without loss of generality, we may assume $T=S$. Since $g$ is a bounded automorphism, there exist $m, m^{\prime} \geq 1$ such that for every $n \geq 1, g(m n T A) \subset n T A$ and $g(n T A) \supset m^{\prime} n T A$. Thus,

$$
\mathscr{H}^{\rho^{g}(\hat{\tau}(m n T A))} \supset \mathscr{H}^{\rho(\hat{\tau}(n T A))} \text { and } \mathscr{H}^{\rho^{g}(\hat{\tau}(n T A))} \subset \mathscr{H}^{\rho\left(\hat{\tau}\left(m^{\prime} n T A\right)\right)} .
$$

Therefore, $\rho^{g}$ is smooth and admissible. Further, $\rho^{g}$ is irreducible since any $\widehat{\mathscr{G}}(L)$ subrepresentation of $\rho^{g}$ is also a $\widehat{\mathscr{G}}(L)$-subrepresentation of $\rho$, which is irreducible. Part (i) follows from Proposition 4.19.

Corollary 4.17 shows that $\rho^{g} \simeq \rho \otimes_{\mathcal{O}_{S}} \mathcal{N}$ as $\widehat{\mathscr{G}}(L)$-representations for some invertible sheaf $\mathcal{N}$ on $S$ (equipped with trivial $\widehat{\mathscr{G}}(L)$-action). The isomorphism provides $f: \mathscr{H} \simeq \mathscr{H} \otimes \mathcal{N}$ as $\mathscr{O}_{S}$-modules. But $f$ obviously induces an isomorphism $\rho \simeq \rho \otimes \mathcal{N}$ of $\widehat{\mathscr{G}}(L)$-representations. Therefore, $\rho^{g} \simeq \rho$.

Now define a group functor $\mathrm{Mp}^{\mathrm{b}}\left(V A, \hat{e}^{L}\right)$ on $(\mathrm{Sch} / S)$ such that for locally noetherian $T$,

$\underline{\operatorname{Mp}}^{\mathrm{b}}\left(V A, \hat{e}^{L}\right)(T)$

$$
=\left\{(g, M) \in \underline{\mathrm{Sp}}^{\mathrm{b}}\left(V A \times T, \hat{e}^{L}\right) \times \underline{\mathrm{Aut}}_{\mathrm{O}_{T}}\left(\mathscr{H} \otimes \mathrm{O}_{T}\right) \mid M \circ \rho_{T} \circ M^{-1}=\rho_{T}^{g}\right\}
$$

with group law $\left(g_{1}, M_{1}\right)\left(g_{2}, M_{2}\right)=\left(g_{1} g_{2}, M_{1} M_{2}\right)$. (The definition is understood as a functor of points.) Similarly define $\underline{\mathrm{Mp}}^{\mathrm{b}}\left(T A, \hat{e}^{L}\right)$ with $T A$ in place of VA. There 
is a sequence of group functors

$$
1 \rightarrow \mathbb{G}_{m} \rightarrow \underline{\mathrm{Mp}}^{\mathrm{b}}\left(V A, \hat{e}^{L}\right) \rightarrow \underline{\mathrm{Sp}}^{\mathrm{b}}\left(V A, \hat{e}^{L}\right) \rightarrow 1 .
$$

The first map $\mathbb{G}_{m} \rightarrow \operatorname{Mp}^{\mathrm{b}}\left(V A, \hat{e}^{L}\right)$ is given by $\alpha \mapsto(1, \alpha)$ using the canonical isomorphism $\mathbb{G}_{m} \simeq$ Aut $_{\mathbb{O}_{S}}(\mathscr{H})$, and the next map sends $(g, M)$ to $g$.

We define variants $\underline{\operatorname{Sp}}\left(V A, \hat{e}^{L}\right)$ and $\underline{\mathrm{Mp}}\left(V A, \hat{e}^{L}\right)$, which are also group functors on $(\mathrm{Sch} / S)$ and (LocNoeth $/ S)$, respectively. For an $S$-scheme $T$, write $T=\prod_{i \in I} T_{i}$ as a disjoint union of connected components. Set

$$
\underline{\operatorname{Sp}}\left(V A, \hat{e}^{L}\right)(T):=\prod_{i \in I} \underline{\operatorname{Sp}}^{\mathrm{b}}\left(V A, \hat{e}^{L}\right)\left(T_{i}\right)
$$

and similarly for $\mathrm{Mp}\left(V A, \hat{e}^{L}\right)(T)$. By the paragraph above Lemma 3.4, The boundedness condition is vacuous in $\underline{\operatorname{Sp}}^{\mathrm{b}}\left(V A, \hat{e}^{L}\right)\left(T_{i}\right)$. As the analogue of (5-3), we have

$$
1 \rightarrow \mathbb{G}_{m} \rightarrow \underline{\mathrm{Mp}}\left(V A, \hat{e}^{L}\right) \rightarrow \underline{\mathrm{Sp}}\left(V A, \hat{e}^{L}\right) \rightarrow 1 .
$$

Remark 5.2. In general, we do not address the issue of representability of $\mathrm{Sp}, \mathrm{Mp}$, $\mathrm{Sp}^{\mathrm{b}}$ and $\mathrm{Mp}^{\mathrm{b}}$ by ind-group schemes. When there is a level structure (Section 6 ),

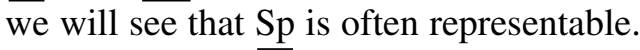

Lemma 5.3. For any locally noetherian $S$-scheme $T$, the sequence of groups $o b$ tained from (5-3) by taking T-points is exact. The same is true for (5-4).

Proof. It is enough to deal with (5-3), which implies the other case easily. The lemma is obvious except for the surjectivity, which we check now. Let $g \in \underline{\operatorname{Sp}}\left(V A, \hat{e}^{L}\right)(T)$. It suffices to show that $\underline{\operatorname{Hom}}_{\widehat{G}\left(L_{T}\right)}\left(\rho_{T}, \rho_{T}^{g}\right)$ has a $T$-point. Since $\rho_{T} \simeq \rho_{T}^{g}$ by the preceding lemma, we have a (noncanonical) isomorphism $\operatorname{Hom}_{\widehat{G}\left(L_{T}\right)}\left(\rho_{T}, \rho_{T}^{g}\right) \simeq$ $\operatorname{Aut}_{\left.\widehat{G}_{(L}\right)}\left(\rho_{T}\right)$. The latter is isomorphic to $\mathbb{G}_{m}(T)$ by Theorem 4.15 , which is certainly nonempty.

Remark 5.4. In the classical analogue of (5-3) (or (5-4)), the exactness in the middle results from the irreducibility of the Heisenberg representation and Schur's lemma. The surjectivity results from the Stone-von Neumann theorem.

Definition 5.5. The tautological representations $\underline{\operatorname{Mp}}^{\mathrm{b}}\left(V A, \hat{e}^{L}\right) \rightarrow \underline{\operatorname{Aut}}_{\mathscr{O}_{S}}(\mathscr{H})$ and $\mathrm{Mp}\left(V A, \hat{e}^{L}\right) \rightarrow{\underline{\mathrm{Aut}_{\mathrm{O}_{S}}}}(\mathscr{H})$, respectively, given as a morphism of group functors on (LocNoeth $/ S)$ by $(g, M) \mapsto M$ is called the Weil representation or the oscillator representation (cf. Remark 2.10).

For the rest of Section 5, we mostly focus on $\mathrm{Mp}^{\mathrm{b}}$ and $\mathrm{Sp}^{\mathrm{b}}$. The results carry over to $\mathrm{Mp}$ and Sp easily (Section $5 \mathrm{E}$ ). The Weil representation is independent of the choice of the Heisenberg representation $\mathscr{H}$ in a suitable sense, as we will soon see. 
Lemma 5.6. If $\underline{\mathrm{Mp}}_{\mathscr{H}}^{\mathrm{b}}\left(V A, \hat{e}^{L}\right)$ and $\mathrm{Mp}_{\mathscr{H}}^{\mathrm{b}}\left(V A, \hat{e}^{L}\right)$ denote the group functors arising from Heisenberg representations $\mathscr{H}^{\prime}$ and $\mathscr{H}^{\prime}$, respectively, then there is a canonical isomorphism of metaplectic group functors sitting in a commutative diagram below:

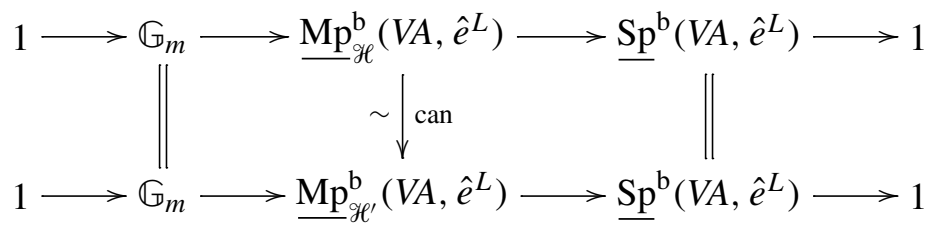

Proof. By Corollary 4.17, $\mathscr{H}^{\prime}=\mathscr{H} \otimes \mathcal{N}$ for an invertible $\mathcal{O}_{S}$-module $\mathcal{N}$. Thus, there

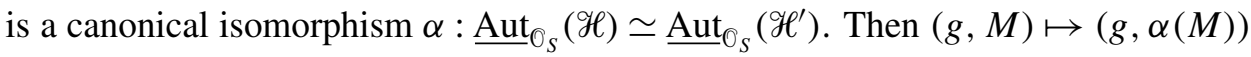
clearly induces the desired isomorphism.

Corollary 5.7. With the notation in the previous lemma, we have the following commutative diagram:

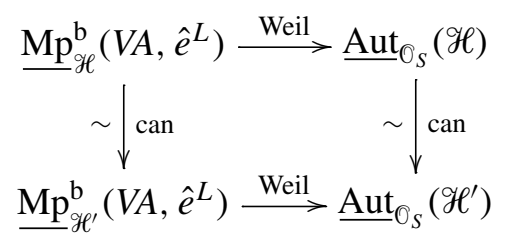

Proof. This result follows immediately from the proof of Lemma 5.6.

We would like to find a splitting of (5-3) over an "open compact subgroup" of $\underline{\operatorname{Sp}}^{\mathrm{b}}\left(V A, \hat{e}^{L}\right)$. Let $m, n \geq 1$. Let $\underline{\mathrm{Sp}}^{\mathrm{b}}\left(\frac{1}{m} T(A, L) ; n T A, \hat{e}^{L}\right)$ denote the subgroup functor of $\mathrm{Sp}^{\mathrm{b}}\left(V A, \hat{e}^{L}\right)$ consisting of $g$ that stabilizes $\frac{1}{m} T(A, L)$ and $n T A$ and induces the identity map on $\frac{1}{m} T(A, L) / n T A$. Note that $\underline{\operatorname{Sp}}^{b}\left(\frac{1}{m} T(A, L) ; n T A, \hat{e}^{L}\right)=$ $\mathrm{Sp}^{\mathrm{b}}\left(T(A, L) ; m n T A, \hat{e}^{L}\right)$. (We will favor the expression on the left-hand side when it seems conceptually helpful.) Now suppose that $(g, M) \in \operatorname{Mp}^{\mathrm{b}}\left(V A, \hat{e}^{L}\right)$ with

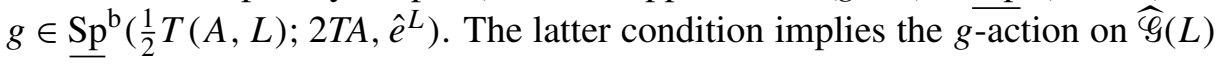

- preserves $\hat{\tau}(2 T A)$, which is equal to $\hat{\sigma}(2 T A)$, and

- leaves $\widetilde{G}\left(2^{*} L\right)$ stable and induces the identity map on

$$
\mathscr{G}\left(2^{*} L\right) \simeq \widetilde{\mathscr{G}}\left(2^{*} L\right) / \hat{\tau}(2 T A) .
$$

By restriction, $M$ induces an isomorphism of representations

$$
M_{0}:\left(\rho \mid \widetilde{\mathscr{G}}_{\left(2^{*} L\right)}, \mathscr{H}^{\hat{\sigma}(2 T A)}\right) \simeq\left(\left.\rho^{g}\right|_{\left.\widetilde{G}_{(2 *} L\right)}, \mathscr{H}^{\hat{\sigma}(2 T A)}\right) .
$$

The representations factor through the quotient $\mathscr{G}\left(2^{*} L\right)$ of $\widetilde{\mathscr{G}}\left(2^{*} L\right)$. Since $g$ acts as the identity on $\mathscr{G}\left(2^{*} L\right)$, we deduce that $\rho^{g}=\rho$ (not just an isomorphism) as $\mathscr{G}\left(2^{*} L\right)$-representations on $\mathscr{H}^{\hat{\sigma}(2 T A)}$. Hence, by Proposition 2.12(iv),

$$
M_{0} \in \underline{\operatorname{Aut}}_{\mathscr{G}_{(L)}}\left(\mathscr{H}^{\hat{\sigma}(2 T A)}\right) \simeq \underline{\operatorname{Aut}}_{\mathbb{O}_{S}}\left(\mathrm{O}_{S}\right) \simeq \mathbb{G}_{m} .
$$


The former of the two canonical isomorphisms above is given by Proposition 2.12(ii). In light of (5-5), there is a unique choice of $M$ (when $g$ is fixed) that restricts to $M_{0}$. This leads to our next result.

Lemma 5.8. There is a canonical splitting of (5-3) over $\operatorname{Sp}^{\mathrm{b}}\left(T A, \hat{e}^{L}\right)$. Namely, there is a map of group functors

$$
\mathrm{spl}: \underline{\mathrm{Sp}}^{\mathrm{b}}\left(\frac{1}{2} T(A, L) ; 2 T A, \hat{e}^{L}\right) \rightarrow \underline{\mathrm{Mp}}^{\mathrm{b}}\left(T A, \hat{e}^{L}\right)
$$

such that if $\operatorname{spl}(g)=\left(g, M_{g}\right)$, then $M_{g}$ corresponds to the identity of $\mathbb{G}_{m}$ via (5-5).

Proof. Let $\alpha: T \rightarrow S$ be an $S$-scheme. For each $g \in \underline{\operatorname{Sp}}^{\mathrm{b}}\left(\frac{1}{2} T(A, L) ; 2 T A, \hat{e}^{L}\right)$, let us define $M_{g}$. As was seen in the proof of Lemma 5.3, there exists $M_{g}^{\prime}$ such that $\left(g, M_{g}^{\prime}\right) \in \mathrm{Mp}^{\mathrm{b}}\left(T A, \hat{e}^{L}\right)(T)$. Such an $M_{g}^{\prime}$ defines an automorphism $a \in \mathbb{G}_{m}(T)$ by (5-5). Set $\overline{M_{g}}:=a^{-1} \cdot M_{g}^{\prime}$. Then $\left(g, M_{g}\right) \in \mathrm{Mp}^{\mathrm{b}}\left(V A, \hat{e}^{L}\right)(T)$, and $M_{g}$ corresponds to $1 \in \mathbb{G}_{m}(T)$ via (5-5). Moreover, it is straightforward to verify $\left(g_{1} g_{2}, M_{g_{1} g_{2}}\right)=$ $\left(g_{1} g_{2}, M_{g_{1}} M_{g_{2}}\right)$ as the images of $M_{g_{1} g_{2}}$ and $M_{g_{1}} M_{g_{2}}$ in $\mathbb{G}_{m}$ via (5-5) are both 1 .

Corollary 5.9. Suppose that $\operatorname{deg} L=1$. Then there is a canonical splitting of (5-3) over $\underline{\operatorname{Sp}}^{\mathrm{b}}\left(T A, 4 T A, \hat{e}^{L}\right)$.

Proof. Immediate, since $T(A, L)=T A$ and

$$
\underline{\mathrm{Sp}}^{\mathrm{b}}\left(T A, 4 T A, \hat{e}^{L}\right)=\underline{\operatorname{Sp}}^{\mathrm{b}}\left(\frac{1}{2} T A, 2 T A, \hat{e}^{L}\right) .
$$

5B. Dual Weil representations. The dual Heisenberg representation $\mathscr{H}^{\vee}$ also plays the role of the dual Weil representation. Namely, $\underline{\operatorname{Mp}}^{\mathrm{b}}\left(A, \hat{e}^{L}\right)$ acts on $\mathscr{H}^{\vee}$ by the rule

$$
\left((g, M) \cdot v^{\vee}\right)(v)=v^{\vee}\left(M^{-1} v\right), \quad v \in \mathscr{H}, v^{\vee} \in \mathscr{H}^{\vee} .
$$

5C. An application of the Künneth formula, Part II. We continue Section 4E with the same notation as in that subsection. Note that there is an obvious embedding

$$
\underline{\mathrm{Sp}}^{\mathrm{b}}\left(A_{1}, \hat{e}^{L_{1}}\right) \times \underline{\mathrm{Sp}}^{\mathrm{b}}\left(A_{2}, \hat{e}^{L_{2}}\right) \hookrightarrow \underline{\operatorname{Sp}}^{\mathrm{b}}\left(A, \hat{e}^{L}\right) .
$$

The following is the analogue of a classical result [Mœglin et al. 1987, II.1(6)]:

Lemma 5.10. The isomorphism

$$
i: \widehat{\mathscr{V}}\left(L_{1}\right) \otimes \widehat{\mathscr{V}}\left(L_{2}\right) \simeq \widehat{\mathscr{V}}(L)
$$

of (4-12) is an isomorphism of $\mathrm{Mp}^{\mathrm{b}}\left(V A_{1}, \hat{e}^{L_{1}}\right) \times \mathrm{Mp}^{\mathrm{b}}\left(V A_{2}, \hat{e}^{L_{2}}\right)$-representations (the notion of representations as in Remark 2.10) if the action on the right-hand side is pulled back via

$$
\begin{aligned}
& \underline{\operatorname{Mp}}^{\mathrm{b}}\left(V A_{1}, \hat{e}^{L_{1}}\right) \times \underline{\operatorname{Mp}^{\mathrm{b}}\left(V A_{2}, \hat{e}^{L_{2}}\right)} \rightarrow \underline{\operatorname{Mp}^{\mathrm{b}}\left(V A, \hat{e}^{L}\right)} \\
&\left(\left(g_{1}, M_{1}\right),\left(g_{2}, M_{2}\right)\right) \mapsto\left(g_{1} \otimes g_{2}, i\left(M_{1} \otimes M_{2}\right) i^{-1}\right) .
\end{aligned}
$$


Proof. This is a tautology in view of the way the metaplectic group action is defined.

5D. Local Weil representations. Let $\rho_{p}: \widehat{\mathscr{G}}_{p}(L) \rightarrow \underline{\operatorname{Aut}}_{\mathbb{O}_{S}}\left(\mathscr{H}_{p}\right)$ be a $p$-adic Heisenberg representation. As in Section 5A, we define $\underline{\operatorname{Mp}}^{\mathrm{b}}\left(V_{p} A, \hat{e}_{p}^{L}\right)$ and $\underline{\operatorname{Sp}}^{\mathrm{b}}\left(V_{p} A, \hat{e}_{p}^{L}\right)$ and fit them into a sequence (cf. (5-3)) that is exact in the sense of Lemma 5.3:

$$
1 \rightarrow \mathbb{G}_{m} \rightarrow \underline{\operatorname{Mp}}^{\mathrm{b}}\left(V_{p} A, \hat{e}_{p}^{L}\right) \rightarrow \underline{\operatorname{Sp}}^{\mathrm{b}}\left(V_{p} A, \hat{e}_{p}^{L}\right) \rightarrow 1 .
$$

The local Weil representation at $p$ is the tautological representation

$$
\underline{\operatorname{Mp}}^{\mathrm{b}}\left(V_{p} A, \hat{e}_{p}^{L}\right) \rightarrow \underline{\operatorname{Aut}}_{\mathbb{O}_{S}}\left(\mathscr{H}_{p}\right) .
$$

There is a splitting of (5-6) over $\underline{\operatorname{Sp}}^{\mathrm{b}}\left(T_{p}(A, L), T_{p} A, \hat{e}_{p}^{L}\right)$ if $p \neq 2$ and over $\underline{\operatorname{Sp}}^{\mathrm{b}}\left(\frac{1}{2} T_{p}(A, L) ; 2 T_{p} A, \hat{e}^{L}\right)$ if $p=2$ (cf. Lemma 5.8). Natural questions on the structure of $\underline{\mathrm{Mp}}^{\mathrm{b}}\left(V_{p} A, \hat{e}_{p}^{L}\right)$ are:

(i) When is (5-6) split?

(ii) If (5-6) is not split, does it come from a double cover? Namely, can we show that $\mathrm{Mp}^{\mathrm{b}}\left(V_{p} A, \hat{e}_{p}^{L}\right)$ has a subgroup functor $\widetilde{\mathrm{Sp}}^{\mathrm{b}}\left(V_{p} A, \hat{e}_{p}^{L}\right)$ that is an extension of $\underline{\mathrm{Sp}}^{\mathrm{b}}\left(V_{p} A, \hat{e}_{p}^{L}\right)$ by $\mu_{2}$ ?

For the classical $p$-adic metaplectic group, it is known that the answers to (i) and (ii) are "never" and "yes", respectively, at least when $p \neq 2$. The questions seem subtle if $S$ is an $\mathbb{F}_{p}$-scheme and already when $S=\operatorname{Spec} \overline{\mathbb{F}}_{p}$. We will see a positive answer to (i) when $A$ is an ordinary abelian variety (Corollary 7.7). We do not have a clue to (ii). See Example 6.9 for the case of supersingular abelian varieties.

5E. From $\mathrm{Mp}^{\mathrm{b}}$ to $\mathbf{M p}$. Most results of Section 5 have been stated about $\mathrm{Mp}^{\mathrm{b}}$ and $\underline{\mathrm{Sp}}^{\mathrm{b}}$. Everything we have proved or asked about $\underline{\mathrm{Mp}}^{\mathrm{b}}$ and $\underline{\mathrm{Sp}}^{\mathrm{b}}$ applies to $\overline{\mathrm{Mp}}$ and $\overline{\mathrm{Sp}}$. The proof is easily reduced to the case of connected base schemes, in which $\overline{c a s e} \underline{M p}^{b}$ and $\underline{M p}$ coincide as well as $\underline{S p}^{b}$ and $\underline{S p}$.

\section{Level structures}

In our context, a level structure is a trivialization of $V A, V_{p} A$ and so on. This allows us to compare our theory with the representation theory of the usual symplectic and metaplectic groups over number fields and $p$-adic fields (which are defined independently of abelian schemes and line bundles). This resembles the level structure arising naturally in the moduli-theoretic setting. It is interesting to note new characteristic $p$ phenomena, which are not observed in the classical theory of Weil representations, when studying the Weil representation of a $p$-adic metaplectic group in characteristic $p$ (Section 6C). Throughout Section 6, we assume that $S$ is locally noetherian. 
6A. Level structure on VA. Let $S$ be a $\mathbb{Q}$-scheme and $(V,\langle\cdot, \cdot\rangle)$ be an evendimensional $\mathbb{Q}$-vector space with a symplectic pairing. Let $\psi: \mathbb{A}^{\infty} \rightarrow \mathbb{G}_{m}$ be a nontrivial morphism of (ind-)group schemes over $S$. (This is the analogue of the additive character in the classical setting.) By composing, we obtain

$$
\langle\cdot, \cdot\rangle_{\psi}: V \otimes \mathbb{A}^{\infty} \times V \otimes \mathbb{A}^{\infty} \rightarrow \mathbb{G}_{m} .
$$

Suppose that there is an isomorphism of ind-group schemes over $S$

$$
\eta: V \otimes \mathbb{A}^{\infty} \simeq V A
$$

that carries $\langle\cdot, \cdot\rangle_{\psi}$ to $\hat{e}^{L}$. This forces $\psi$ to factor through $\mu_{\infty} \hookrightarrow \mathbb{G}_{m}$ since $\hat{e}^{L}$ factors through $\mu_{\infty} \hookrightarrow \mathbb{G}_{m}$ (Lemma 3.17).

Lemma 3.18 together with $\eta$ allows us to identify $\mathbb{G}_{m} \times\left(V \otimes \mathbb{A}^{\infty}\right) \simeq \widehat{\mathscr{G}}(L)$, where the left-hand side, to be denoted $\widehat{\mathscr{G}}\left(V,\langle\cdot, \cdot\rangle_{\psi}\right)$, has group law

$$
(\lambda, x) \cdot(\mu, y)=\left(\lambda \mu \cdot\langle x / 2, y\rangle_{\psi}, x+y\right) .
$$

Again via $\eta$, the exact sequences in (3-5) and (5-4) become

$$
\begin{gathered}
1 \rightarrow \mathbb{G}_{m} \rightarrow \widehat{\mathscr{G}}\left(V,\langle\cdot, \cdot\rangle_{\psi}\right) \rightarrow V \otimes \mathbb{A}^{\infty} \rightarrow 0, \\
1 \rightarrow \mathbb{G}_{m} \rightarrow \underline{\operatorname{Mp}}\left(V \otimes \mathbb{A}^{\infty},\langle\cdot, \cdot\rangle_{\psi}\right) \rightarrow \underline{\operatorname{Sp}}\left(V \otimes \mathbb{A}^{\infty},\langle\cdot, \cdot\rangle_{\psi}\right) \rightarrow 1 .
\end{gathered}
$$

(The analogue for $\mathrm{Mp}^{\mathrm{b}}$ and $\mathrm{Sp}^{\mathrm{b}}$ is also obtained from (5-3).) The group functor $\operatorname{Sp}\left(V \otimes \mathbb{A}^{\infty},\langle\cdot, \cdot\rangle \bar{\psi}\right)$ is represented by the constant group scheme associated with the usual symplectic group $\operatorname{Sp}\left(V \otimes \mathbb{A}^{\infty},\langle\cdot, \cdot\rangle_{\psi}\right)$ while Mp is defined by the same recipe as in (5-2) (using $\underline{S p}$ in place of $\underline{S p}^{b}$ ).

Remark 6.1. Note that $\eta$ does not exist unless $S$ is in characteristic 0 because $V_{p} A$ is not a constant ind-group scheme at any point $s \in S$ of residue characteristic $p$. See Section $6 \mathrm{C}$ for a different kind of level structure.

Remark 6.2. In the simple case when $S=\operatorname{Spec} k$ and $k$ is an algebraically closed field of characteristic 0 , a choice of $\chi: \mathbb{Q} / \mathbb{Z} \stackrel{\sim}{\rightarrow} \mu_{\infty}$ over $k$ gives rise to $\psi$ in the following manner:

$$
\psi: \mathbb{A}^{\infty} \rightarrow \mathbb{A}^{\infty} / \widehat{\mathbb{Z}} \stackrel{\text { can }}{\simeq} \mathbb{Q} / \mathbb{Z} \stackrel{\chi}{\simeq} \mu_{\infty} \hookrightarrow \mathbb{G}_{m} .
$$

6B. Local level structure, Part I. We consider two kinds of level structures on $V_{p} A$. The first one is the local analogue of Section 6A. Let $\left(V_{p},\langle\cdot, \cdot\rangle\right)$ be a symplectic $\mathbb{Q}_{p}$-vector space and $\psi: \mathbb{Q}_{p} \rightarrow \mathbb{G}_{m}$ a nontrivial morphism of (ind-)group schemes over $S$. Thereby, obtain $\langle\cdot, \cdot\rangle_{\psi}: V_{p} \times V_{p} \rightarrow \mathbb{G}_{m}$, where $V_{p}$ is also viewed as a constant ind-group scheme over $S$. A level structure is a $\mathbb{Q}_{p}$-linear isomorphism

$$
\eta: V_{p} \simeq V_{p} A
$$


$\left(\mathbb{Q}_{p}\right.$ acts on $V_{p} A$ as explained in Section $3 \mathrm{~A}$ ) carrying $\langle\cdot, \cdot\rangle_{\psi}$ to $\hat{e}_{p}^{L}$. As in Section 6A, this forces $\psi$ to factor through $\mu_{p^{\infty}} \hookrightarrow \mathbb{G}_{m}$. The map $\eta$ and the $p$-adic analogue of Lemma 3.18 enable us to identify $\widehat{\mathscr{G}}_{p}\left(V_{p},\langle\cdot, \cdot\rangle_{\psi}\right):=\mathbb{G}_{m} \times V_{p}$ with $\widehat{\mathscr{G}}_{p}(L)$, where the former is equipped with the same group law as in (6-1). We obtain exact sequences

$$
\begin{aligned}
1 & \rightarrow \mathbb{G}_{m} \rightarrow \widehat{\mathscr{G}}_{p}\left(V_{p},\langle\cdot, \cdot\rangle_{\psi}\right) \rightarrow V_{p} \rightarrow 0, \\
1 \rightarrow \mathbb{G}_{m} & \rightarrow \underline{\operatorname{Mp}}\left(V_{p},\langle\cdot, \cdot\rangle_{\psi}\right) \rightarrow \underline{\operatorname{Sp}}\left(V_{p},\langle\cdot, \cdot\rangle_{\psi}\right) \rightarrow 1 .
\end{aligned}
$$

6C. Local level structure, Part II. When $S$ is in characteristic $p$, a different level structure is desirable (cf. Remark 6.1). Let $k$ be a field extension of $\mathbb{F}_{p}$. Suppose that $S$ is a $k$-scheme. Let $\left(\Sigma,\langle\cdot, \cdot\rangle_{0}\right)$ be a $p$-divisible group $\Sigma$ over $k$ with an alternating pairing $\langle\cdot, \cdot\rangle_{0}: \Sigma \times \Sigma \rightarrow \mu_{p^{\infty}}$. This can be promoted to

$$
\langle\cdot, \cdot\rangle_{1}: V_{p} \Sigma \times V_{p} \Sigma \rightarrow V_{p} \mu_{p^{\infty}}
$$

by the functoriality of $V_{p}$. Let $b_{p}: V_{p} \mu_{p^{\infty}} \rightarrow \mu_{p^{\infty}}$ be the $p$-adic analogue of $b$ in Section 3D. Set $\langle\cdot, \cdot\rangle:=b_{p} \circ\langle\cdot, \cdot\rangle_{1}$. Then a level structure is a $\mathbb{Q}_{p}$-linear isomorphism

$$
\zeta: V_{p} \Sigma \times_{k} S \stackrel{\sim}{\rightarrow} V_{p} A
$$

matching $\langle\cdot, \cdot\rangle$ and $\hat{e}_{p}^{L}$. Set $\widehat{\mathscr{G}}_{p}\left(\Sigma,\langle\cdot, \cdot\rangle_{0}\right):=\mathbb{G}_{m} \times V_{p} \Sigma$ with the group law

$$
(\lambda, x) \cdot(\mu, y)=\left(\lambda \mu \cdot\left\langle\frac{1}{2} x, y\right\rangle, x+y\right) .
$$

In light of the $p$-adic analogue of Lemma 3.18, $\zeta$ induces an isomorphism

$$
\widehat{\mathscr{G}}_{p}\left(\Sigma,\langle\cdot, \cdot\rangle_{0}\right) \simeq \widehat{\mathscr{G}}_{p}(L) \text {. }
$$

The $p$-adic analogues of exact sequences in (3-5) and (5-4) are identified via $\zeta$ with the following, where Mp and Sp are defined as in Section 5A:

$$
\begin{aligned}
1 & \rightarrow \mathbb{G}_{m} \rightarrow \widehat{\widehat{G}}_{p}\left(\Sigma,\langle\cdot, \cdot\rangle_{0}\right) \rightarrow V_{p} \Sigma \rightarrow 0, \\
1 \rightarrow \mathbb{G}_{m} & \rightarrow \underline{\operatorname{Mp}}\left(V_{p} \Sigma,\langle\cdot, \cdot\rangle\right) \rightarrow \underline{\operatorname{Sp}}\left(V_{p} \Sigma,\langle\cdot, \cdot\rangle\right) \rightarrow 1 .
\end{aligned}
$$

A priori $\operatorname{Mp}\left(V_{p} \Sigma,\langle\cdot, \cdot\rangle\right)$ depends not only on $\left(\Sigma,\langle\cdot, \cdot\rangle_{0}\right)$ but also on $(A, L)$ because the definition involves the Heisenberg representation, which is constructed from $(A, L)$. But Corollary 4.17 shows that two Heisenberg representations of $\widehat{\mathscr{G}}_{p}\left(\Sigma,\langle\cdot, \cdot\rangle_{0}\right)$ (constructed from two choices of $(A, L)$ ) differ by a tensoring with an invertible $O_{S}$-module, so $\operatorname{Mp}\left(V_{p} \Sigma,\langle\cdot, \cdot\rangle\right)$ and its Weil representation depend (up to isomorphism) only on $\left(\Sigma,\langle\cdot, \cdot\rangle_{0}\right)$ thanks to Corollary 5.7.

Remark 6.3. One can consider a variant when $S$ is not entirely in characteristic $p$. For instance, if $\Sigma=\left(\mathbb{Q}_{p} / \mathbb{Z}_{p} \times \mu_{p^{\infty}}\right)^{g}$ for some $g \geq 1$, which can be defined (together with $\langle\cdot, \cdot\rangle_{0}$ ) over Spec $\mathbb{Z}$, one can take $S$ to be any locally noetherian 
scheme, and the construction above goes through. On the other hand, if $\left(\Sigma,\langle\cdot, \cdot\rangle_{0}\right)$ is as above except that the base ring is not $k$ but the integer ring $\mathcal{O}$ in an algebraic extension field of $\mathbb{Q}_{p}$, the discussion can be adapted to any $\mathrm{O}$-scheme $S$.

Remark 6.4. The level structure $\zeta$ is the analogue of the Igusa level structure used in the literature (e.g., [Katz and Mazur 1985; Harris and Taylor 2001; Hida 2004]).

Remark 6.5. It is an interesting phenomenon that the Heisenberg group and the metaplectic group at $p$ heavily depend on the isogeny type of $\Sigma$ (or $A\left[p^{\infty}\right]$ ) when $S$ is in characteristic $p$. This is evident in (6-4), for instance. Each isogeny type gives rise to a different mod $p$ Weil representation.

6D. Weil representations associated with p-divisible groups, without abelian varieties. Assume $p \neq 2$. Let $\left(\Sigma,\langle\cdot, \cdot\rangle_{0}\right)$ and $\langle\cdot, \cdot\rangle$ be as in Section $6 \mathrm{C}$ with $k=\overline{\mathbb{F}}_{p}$. For simplicity, assume that $\langle\cdot, \cdot\rangle_{0}$ is a perfect pairing. (In general it is enough to require $\langle\cdot, \cdot\rangle$ to be a perfect pairing.) We know that there exists an $(A, L)$ such that there is a symplectic isomorphism $\zeta: V_{p} \Sigma \simeq V_{p} A$ thanks to Oort's result ([Rapoport 2005, Theorem 7.4], cf. [Oort 2001]) that any Newton polygon stratum in the $\bmod p$ fiber of the Siegel modular variety with hyperspecial level at $p$ is nonempty. Then Section $6 \mathrm{C}$ attaches the Heisenberg group/representation and Weil representation to $\left(\Sigma,\langle\cdot, \cdot\rangle_{0}\right)$. The goal of this subsection is to sketch an alternative approach without using $(A, L)$ at all.

Recall that $\widehat{\mathscr{G}}_{p}:=\widehat{\mathscr{G}}_{p}\left(\Sigma,\langle\cdot, \cdot\rangle_{0}\right)$ is already defined in Section 6C independently of $(A, L)$. The key point will be to prove the existence of the Heisenberg representation of $\widehat{\mathscr{G}}_{p}$ without resorting to $(A, L)$. In particular, we use the fact that any nondegenerate theta group possesses a weight-1 irreducible representation over an algebraically closed field [Moret-Bailly 1985, Chapter 5, Theorem 2.5.5].

Take $\hat{\sigma}_{p}: T_{p} \Sigma \rightarrow \widehat{G}_{p}\left(\Sigma,\langle\cdot, \cdot\rangle_{0}\right)$ to be the natural embedding $x \mapsto(1, x)$. (The assumption $p \neq 2$ is used to ensure that the latter embedding preserves group structure.) It is easy to verify the analogue of Lemma 3.15 for $\widehat{\mathscr{G}}_{p}, \hat{\sigma}_{p}$, etc. (replacing $\widetilde{\mathscr{G}}\left(\left(p^{n}\right)^{*} L\right)$ there with $\widetilde{\mathscr{G}}\left(p^{n}\right):=\mathbb{G}_{m} \times \frac{1}{p^{n}} T_{p} \Sigma$ in $\left.\widehat{\mathscr{G}}_{p}\right)$, in which $\hat{\sigma}_{p}\left(T_{p} \Sigma\right)$ embeds via the map $1 \times p^{n}$. Set $\mathscr{G}\left(p^{n}\right):=\widetilde{\mathscr{G}}\left(p^{n}\right) / \hat{\sigma}_{p}\left(T_{p} \Sigma\right)$, which is isomorphic to $\mathbb{G}_{m} \times \Sigma\left[p^{2 n}\right]$ (which inherits the twisted group law). By the theorem of [Moret-Bailly 1985] cited above, each $\mathscr{G}\left(p^{n}\right)$ possesses a Heisenberg representation (irreducible representation over $k$ of dimension $p^{n}$ ) for $n \geq 1$. The Heisenberg representation $\mathscr{H}$ of $\widehat{\mathscr{G}}_{p}$ is obtained by patching via the analogue of Lemma 4.5, and then one can check the analogues of Theorem 4.15, Proposition 4.19 and Corollaries 4.26 and 4.28. (Of course $T A, V A$ and $\hat{e}^{L}$ should be replaced by $T_{p} \Sigma, V_{p} \Sigma$, and $\langle\cdot, \cdot\rangle$, and $e_{*}^{L}$ should be ignored.) The construction of Section $5 \mathrm{~A}$ carries over to $\underline{\operatorname{Mp}}\left(V_{p} \Sigma,\langle\cdot, \cdot\rangle_{0}\right)$ and its Weil representation on $\mathscr{H}$.

Remark 6.6. What we have denoted $\hat{\sigma}_{p}$ should be thought of as the analogue of $\hat{\tau}_{p}$ in the previous sections (although there is no distinction when $p \neq 2$ ). Perhaps 
one can still work with $p=2$ if we select $e_{*}: \Sigma[2] \times \Sigma[2] \rightarrow \mu_{2}$, satisfying the properties of Lemma 3.19, to play the role of $e_{*}^{L}$. Then the above definition of $\hat{\sigma}_{2}$ should be multiplied by $e_{*}$ (cf. (3-11)).

Remark 6.7. If $\operatorname{char}(k) \neq p$ and $k=\bar{k}$, then one can identify $V_{p} \Sigma$ with a symplectic $\mathbb{Q}_{p}$-vector space (as a constant group scheme), and the above construction still goes through without $(A, L)$. When $k=\mathbb{C}$, this essentially recovers the classical construction.

Remark 6.8. We have worked with $\Sigma$ over $\overline{\mathbb{F}}_{p}$ rather than over a more general scheme $S$. The only essential reason is that the existence of Heisenberg representations (i.e., the analogue of [Moret-Bailly 1985, Chapter 5, Theorem 2.5.5]) no longer holds in general. A sufficient condition for the existence of a Heisenberg representation is that $\Sigma$ over $S$ comes from some $(A, L)$.

Example 6.9. Let $\Sigma_{1 / 2}$ denote a supersingular $p$-divisible group over $\overline{\mathbb{F}}_{p}$ of height 2 and dimension 1 equipped with a perfect pairing $(\cdot, \cdot): \Sigma_{1 / 2} \times \Sigma_{1 / 2} \rightarrow \mu_{p^{\infty}}$. Let $D_{1 / 2}$ be a central quaternion algebra over $\mathbb{Q}_{p}$ of invariant $1 / 2$. It is well known that End $_{\overline{\mathbb{F}}_{p}}\left(\Sigma_{1 / 2}\right)$ is isomorphic to the maximal order of $D_{1 / 2}$, so $\operatorname{End}_{\overline{\mathbb{F}}_{p}}\left(V \Sigma_{1 / 2}\right) \simeq D_{1 / 2}$. (In general, one can use Dieudonné theory to classify $p$-divisible groups $\Sigma$ over $\overline{\mathbb{F}}_{p}$ up to isogeny and identify $\operatorname{End}_{\overline{\mathbb{F}}_{p}}(V \Sigma)$ as a semisimple $\mathbb{Q}_{p}$-algebra. See any standard reference such as [Demazure 1972].)

Set $\Sigma:=\left(\Sigma_{1 / 2}\right)^{g}$, and define $\langle\cdot, \cdot\rangle: \Sigma \times \Sigma \rightarrow \mu_{p^{\infty}}$ by

$$
\left\langle\left(x_{i}\right)_{i=1}^{g},\left(y_{i}\right)_{i=1}^{g}\right\rangle=\prod_{i=1}^{g}\left(x_{i}, y_{i}\right) .
$$

Then

$$
\underline{\operatorname{Sp}}\left(V_{p} \Sigma,\langle\cdot, \cdot\rangle_{0}\right) \simeq \operatorname{Sp}_{g}\left(D_{1 / 2}\right)
$$

as constant group schemes over $\overline{\mathbb{F}}_{p}$. Observe that this group is an inner form of $\mathrm{Sp}_{2 g}\left(\mathbb{Q}_{p}\right)$. The questions (i) and (ii) of Section 5D would be especially interesting to answer in this case. We would guess "no" to (i) and "yes" to (ii) in this case but without much evidence. The only heuristic reason is that this $\Sigma$ is the unique $p$-divisible group over $\overline{\mathbb{F}}_{p}$ (up to isogeny) that is self-dual and isoclinic, so it makes harder for (5-6) (or the analogous sequence for $\Sigma$ ) to split. (For any other choice of a self-dual $\Sigma$, the group of $\overline{\mathbb{F}}_{p}$-points of $\operatorname{Sp}\left(V_{p} \Sigma,\langle\cdot, \cdot\rangle_{0}\right)$ is isomorphic to the group of $\mathbb{Q}_{p}$-points of an inner form of a proper Levi subgroup of $\operatorname{Sp}_{2 g}\left(\mathbb{Q}_{p}\right)$. This is a well known fact in the theory of isocrystals applied to $\mathrm{Sp}_{2 g}$, where the former group is often denoted $J_{b}\left(\mathbb{Q}_{p}\right)$. See [Kottwitz 1997] for instance.)

6E. Global level structure. It is clear how to put together local level structures to get a global one. Let $\mathbb{A}^{\infty, p}$ be the prime-to- $p$ part of $\mathbb{A}^{\infty}$, namely $\widehat{\mathbb{Z}}^{p}:=\prod_{l \neq p} \mathbb{Z}_{l}$ and $\mathbb{A}^{\infty, p}:=\lim _{\longrightarrow} \frac{1}{n} \widehat{\mathbb{Z}}^{p}$, where $n$ runs over positive integers prime to $p$. When $S$ is a $\mathbb{Q}$-scheme, this is done in the obvious manner by globalizing Section 6B. Let us 
say a few words when $S$ is an $\mathbb{F}_{p}$-scheme. Consider the analogue $\psi^{p}: A^{\infty, p} \rightarrow \mathbb{G}_{m}$ of $\psi$ so that we have $\langle\cdot, \cdot\rangle_{\psi^{p}}: V \otimes \mathbb{A}^{\infty, p} \times V \otimes \mathbb{A}^{\infty, p} \rightarrow \mathbb{G}_{m}$ (cf. Section 6B). Let $\left(\Sigma,\langle\cdot, \cdot\rangle_{0}\right)$ be as in Section $6 \mathrm{C}$, and set $\langle\cdot, \cdot\rangle_{p}:=b_{p} \circ\langle\cdot, \cdot\rangle_{1}$ using notation there. A level structure in this setting is an $\mathbb{A}^{\infty}$-linear isomorphism

$$
\left(\eta^{p}, \zeta\right): V \otimes \mathbb{A}^{\infty, p} \times\left(V_{p} \Sigma \times_{k} S\right) \stackrel{\sim}{\rightarrow} V A
$$

carrying $\left(\langle\cdot, \cdot\rangle_{\psi^{p}},\langle\cdot, \cdot\rangle_{p}\right)$ to $\hat{e}^{L}$. We have exact sequences that look like (6-2) away from $p$ and (6-4) at $p$.

\section{Explicit models}

In the study of Weil representations and the theta correspondence, it is important to find a good model on which the group action can be described explicitly. For $p$-adic or finite adelic metaplectic groups, the most popular models in the classical context are Schrödinger and lattice models. In Section 7, we focus on the $p$-adic setting and describe the models for Heisenberg and Weil representations in some simple cases. In those cases $S$ is local, so the Heisenberg representation is unique up to isomorphism (Corollary 4.18). The mixed characteristic phenomenon of Section 7D is intriguing and begs further investigation.

Throughout Section 7, $L$ is assumed to be symmetric and nondegenerate of degree 1. (The assumption on degree may not be essential but is very convenient. Degree 1 can be achieved over an algebraically closed field for any $(A, L)$ without disturbing symmetry and nondegeneracy if we are allowed to modify $(A, L)$ by an isogeny. See [Mumford 1974, §23, Theorem 4, cf. Corollary 1].) Let $C^{\infty}(\cdot, k)$ and $C_{c}^{\infty}(\cdot, k)$ denote the $k$-vector spaces of locally constant and, respectively, locally constant and compactly supported $k$-valued functions and $D^{\infty}(\cdot, k)$ denote the $k$-vector space dual of $C^{\infty}(\cdot, k)$. Throughout this section, a $k$-valued function is understood without further comments as a sheaf-theoretic homomorphism with target $\mathrm{O}_{\text {Spec } k}$, but note that in the setting of Section 7A, this is no different from a function in the naïve sense.

7A. Over a field of characteristic not equal to $p$. Suppose that $S=\operatorname{Spec} k$, where $k$ is algebraically closed of characteristic unequal to $p$. Therefore, $V_{p} A$ is isomorphic to the constant ind-group scheme $\mathbb{Q}_{p}^{2 g}$ over $S$. In this subsection, we may and will view $V_{p} A$ as a $\mathbb{Q}_{p}$-vector space with symplectic pairing $\hat{e}_{p}^{L}: V_{p} A \times V_{p} A \rightarrow k^{\times}$. Similarly, $T_{p} A$ is regarded as a free $\mathbb{Z}_{p}$-module sitting inside $V_{p} A$.

Corollaries 4.28 and 4.18 , adapted to the local setting, tell us that the lattice model for the dual Heisenberg representation may be described as

$$
\begin{aligned}
\mathscr{H}_{\text {lattice }}^{\vee}=\left\{\phi \in C^{\infty}\left(V_{p} A, k\right)\right. \\
\left.\qquad \phi(x)=e_{*}^{L}\left(\frac{1}{2} y\right) \hat{e}^{L}\left(\frac{1}{2} x, y\right) \cdot \phi(x+y), \forall x \in V_{p} A, y \in T_{p} A\right\}
\end{aligned}
$$


with $(\lambda, z) \in \mathbb{G}_{m} \times V A \simeq \widehat{\mathscr{G}}(L)$ acting as $((\lambda, z) \phi)(x)=\lambda^{-1} \hat{e}^{L}(x / 2, z) \cdot \phi(x-z)$. Note that $e_{*}^{L} \equiv 1$ unless $p=2$ (see Section 3F). The lattice model $\mathscr{H}_{\text {lattice, }}$, the dual of $\mathscr{H}_{\text {lattice }}^{\vee}$, admits a concrete description

$$
\begin{aligned}
& \mathscr{H}_{\text {lattice }}=\left\{\phi \in C_{c}^{\infty}\left(V_{p} A, k\right)\right. \\
& \left.\mid \phi(x)=e_{*}^{L}\left(\frac{1}{2} y\right) \hat{e}^{L}\left(\frac{1}{2} x, y\right)^{-1} \cdot \phi(x+y), \forall x \in V_{p} A, y \in T_{p} A\right\}
\end{aligned}
$$

with the dual action; namely, $(\lambda, z)$ acts as $((\lambda, z) \phi)(x)=\lambda \hat{e}^{L}(z / 2, x) \cdot \phi(x+z)$. Indeed the pairing

$$
\mathscr{H}_{\text {lattice }} \times \mathcal{H}_{\text {lattice }}^{\vee} \rightarrow k, \quad(f, g) \mapsto \sum_{x \in V_{p} A / T_{p} A} f(x) g(x)
$$

is easily verified to be $k$-linear, perfect and $\widehat{\mathscr{G}}(L)$-equivariant. Refer to the literature such as [Mœglin et al. 1987, Chapter 2.II.8] (when $p \neq 2$ ) for a precise description

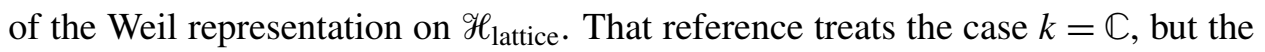
same formula applies if $p \neq \operatorname{char}(k)$.

On the other hand, let $T_{p} A=\Lambda_{1} \oplus \Lambda_{2}$ be a decomposition into free $\mathbb{Z}_{p^{-}}$ submodules that are totally isotropic for $\hat{e}_{p}^{L}$ and in perfect duality with respect to $\hat{e}_{p}^{L}$. Setting $V_{i}=\Lambda_{i} \otimes_{\mathbb{Z}_{p}} \mathbb{Q}_{p}$ for $i=1$, 2, we have $V_{p} A=V_{1} \oplus V_{2}$ and may identify $V_{2}$ with $V_{1}^{\vee}$. When $p=2$, we assume that

$$
\forall x=\left(x_{1}, x_{2}\right) \in \Lambda_{1} \oplus \Lambda_{2}, \quad e_{*}^{L}(x / 2) \hat{e}_{p}^{L}\left(x_{1} / 2, x_{2}\right)=1 .
$$

The above condition amounts to assuming that $L$ is even symmetric in the terminology of [Mumford 2007, Proposition 4.20]. This can always be achieved by pulling back $L$ via the translation $T_{x}$ for a suitable $x \in A[2](k)$. See [Mumford 2007, Corollary 4.24]. The Schrödinger model is (e.g., [Mœglin et al. 1987, Chapter 2.I.4.1], [Mumford 2007, Proposition 5.2.A])

$\mathscr{H}_{\mathrm{Sch}}=C_{c}^{\infty}\left(V_{2}, k\right), \quad\left(\left(\lambda, z_{1}, z_{2}\right) \cdot \phi\right)\left(x_{2}\right)=\lambda \hat{e}^{L}\left(x_{2}, z_{1}\right) \hat{e}^{L}\left(z_{2} / 2, z_{1}\right) \cdot \phi\left(x_{2}+z_{2}\right)$,

where we write $z=\left(z_{1}, z_{2}\right) \in V_{1} \oplus V_{2}$. Corollary 4.18 ensures that $\mathscr{H}_{\text {lattice }} \simeq \mathscr{H}_{\text {Sch }}$ as $\widehat{\mathscr{G}}(L)$-representations on $k$-vector spaces. Refer to [Mumford 2007, Proposition 5.2], for example, to see an explicit isomorphism. Let us recall an explicit formula for the Weil representation on $\mathscr{H}_{\text {Sch }}$ to be compared to the mod $p$ case later (Section 7C).

Proposition 7.1. Consider $M_{g} \in \underline{\mathrm{Aut}}_{k}\left(\mathscr{H}_{\mathrm{Sch}}\right)$ for $g \in \underline{\mathrm{Sp}}\left(V_{p} A, \hat{e}_{p}^{L}\right)$ in the following three cases. (Here $M_{g}$ and $g$ are implicitly $T$-valued points for a locally noetherian $k$-scheme $T$. The matrices below are written with respect to $V_{p} A \simeq V_{2}^{\vee} \oplus V_{2}$. In (iii), we choose a $k$-valued Haar measure on $V_{2}$, which exists since $p \neq \operatorname{char}(k)$.)

$$
\text { (i) } g=\left(\begin{array}{cc}
{ }^{t} B^{-1} & 0 \\
0 & B
\end{array}\right),\left(M_{g} \phi\right)(x)=|\operatorname{det} B|_{p}^{-1 / 2} \phi\left(B^{-1} x\right) \text { for any } B \in G L_{k}\left(V_{2}\right) \text {. }
$$


(ii) $g=\left(\begin{array}{ll}I & C \\ 0 & I\end{array}\right), \quad\left(M_{g} \phi\right)(x)=\hat{e}_{p}^{L}(C x, x) \phi(x)$, where $C \in \operatorname{Hom}_{k}\left(V_{2}, V_{2}^{\vee}\right)$ is symmetric (i.e., $C=C^{\vee}$ ).

(iii) $g=\left(\begin{array}{rr}0 & I \\ -I & 0\end{array}\right),\left(M_{g} \phi\right)(x)=\int_{V_{2}} \hat{e}_{p}^{L}(x, y) \phi(y) d y$.

Then we have $\left(g, M_{g}\right) \in \underline{\mathrm{Mp}}\left(V_{p} A, \hat{e}_{p}^{L}\right)$ in all three cases.

Proof. This is proved by the same computation as in the proof of [Mumford 2007, Lemma 8.2] (cf. [Mœglin et al. 1987, Chapter 2.II.6]).

Remark 7.2. Classically the factor $|\operatorname{det} B|_{p}^{-1 / 2}$ in (i) is inserted to make $M_{g}$ a unitary operator. Of course $\left(g, M_{g}\right) \in \underline{\mathrm{Mp}}\left(V_{p} A, \hat{e}_{p}^{L}\right)$ still holds if $|\operatorname{det} B|_{p}^{-1 / 2}$ is erased.

Example 7.3. The classical Heisenberg and Weil representations (for $p$-adic groups) are obtained when $k=\mathbb{C}$ and $A=\mathbb{C}^{g} / \Lambda$ with $\Lambda=\mathbb{Z}^{g}+i \mathbb{Z}^{g}$, and $L$ arises from a Riemann form $\Lambda \times \Lambda \rightarrow \mathbb{Z}$ defining a principal polarization.

Remark 7.4. In the definition of $\mathscr{H}_{\mathrm{Sch}}$, one cannot use $C^{\infty}\left(V_{2}, k\right)$ because the latter is not smooth with respect to the $\widehat{\mathscr{G}}(L)$-action (defined by the same formula). As for $\mathscr{H}_{\text {lattice }}, C_{c}^{\infty}$ cannot be replaced by $C^{\infty}$ either for the same reason: the $\widehat{\mathscr{G}}(L)$-action on the $C^{\infty}$-space is not smooth. Likewise, $\mathscr{H}_{\text {lattice }}^{\vee}$ is nonsmooth.

7B. Lattice model over $\overline{\mathbb{F}}_{\boldsymbol{p}}$. Suppose that $S=\operatorname{Spec} k$ with $k=\overline{\mathbb{F}}_{p}$. The dual lattice model $\mathscr{H}_{\text {lattice }}^{\vee}$, which is again unique up to isomorphism, has the same description as Corollary 4.28 (cf. (7-1)). As before, $\mathscr{H}_{\text {lattice }}$ is defined to be the dual of $\mathcal{H}_{\text {lattice }}^{\vee}$ (and equipped with the dual action). Unlike (7-2), we do not have the notion of compact support on $V_{p} A$, so view $\mathscr{H}_{\text {lattice just as a space of distributions. An interesting }}$ problem would be to find an explicit formula for the Weil representation on $\mathscr{H}_{\text {lattice. }}$.

7C. Schrödinger model over $\overline{\mathbb{F}}_{\boldsymbol{p}}$. Let $k=\overline{\mathbb{F}}_{p}$ as before. Unlike lattice models, Schrödinger models do not always exist. The first obstruction is that $V_{p} A$ or $A\left[p^{\infty}\right]$ is not always completely polarizable. For instance, if $A$ is a supersingular elliptic curve, then $A\left[p^{\infty}\right]$ does not admit a product decomposition. According to Dieudonné theory, we can achieve

$$
\zeta: \Sigma_{1} \times \Sigma_{2} \simeq A\left[p^{\infty}\right]
$$

for mutually dual $p$-divisible groups $\Sigma_{1}$ and $\Sigma_{2}$ over $k$, by modifying $A$ with an isogeny if necessary, if there are an exactly even number of simple $p$-divisible groups of slope $\frac{1}{2}$ in $A\left[p^{\infty}\right]$. Let us suppose that this is the case so that (7-5) exists. Also suppose that (7-5) is a complete polarization, i.e., $\left.\hat{e}_{p}^{L}\right|_{\Sigma_{1} \times \Sigma_{1}} \equiv 1$, $\left.\hat{e}_{p}^{L}\right|_{\Sigma_{2} \times \Sigma_{2}} \equiv 1$ and $\hat{e}_{p}^{L}$ defines a perfect pairing between $\Sigma_{1}$ and $\Sigma_{2}$. Then we also have $V_{p} A \simeq V_{p}\left(A\left[p^{\infty}\right]\right) \simeq V_{p} \Sigma_{1} \times V_{p} \Sigma_{2}$. Now that there is a complete polarization, 
one can ask whether there is a Schrödinger model for $\mathscr{H}$. The answer is positive in the simplest case.

Proposition 7.5. Suppose that A is ordinary; in other words, there exists an isomorphism $A\left[p^{\infty}\right] \simeq \Sigma_{1} \times \Sigma_{2}$ with $\Sigma_{1}=\left(\mu_{p^{\infty}}\right)^{g}$ and $\Sigma_{2}=\left(\mathbb{Q}_{p} / \mathbb{Z}_{p}\right)^{g}$. If $p=2$, assume that (7-3) holds with $T_{p} \Sigma_{1} \times T_{p} \Sigma_{2}$ in place of $\Lambda_{1} \oplus \Lambda_{2}$. Then the $k$-vector space $\mathscr{H}_{\text {Sch }}:=C_{c}^{\infty}\left(V_{p} \Sigma_{2}, k\right)$ (where $V_{p} \Sigma_{2}$ is viewed as a $\mathbb{Q}_{p}$-vector space) on which $\left(\lambda, z_{1}, z_{2}\right) \in \mathbb{G}_{m} \times V_{p} \Sigma_{1} \times V_{p} \Sigma_{2} \simeq \widehat{\mathscr{G}}(L)$ acts by

$$
\left(\left(\lambda, z_{1}, z_{2}\right) \cdot \phi\right)\left(x_{2}\right)=\lambda \hat{e}_{p}^{L}\left(x_{2}, z_{1}\right) \hat{e}_{p}^{L}\left(z_{2} / 2, z_{1}\right) \cdot \phi\left(x_{2}+z_{2}\right)
$$

is a Heisenberg representation of $\widehat{\varphi}(L)$.

Remark 7.6. The above formula is the same as (7-4) except that it should be interpreted scheme-theoretically. On the other hand, the lemma does not generalize to the nonordinary case as $C_{c}^{\infty}\left(V_{p} \Sigma_{2}, k\right)$ has no natural meaning if $\Sigma_{2}$ is not étale.

Proof. Without loss of generality, we may assume $\hat{e}_{p}^{L}$ is the standard symplectic pairing (of the form (7-7)). Then it is easily verified that

$$
\mathscr{H}_{\mathrm{Sch}}^{p^{n} T_{p} \Sigma}=C^{\infty}\left(\frac{1}{p^{n}} T_{p} \Sigma_{2} / p^{n} T_{p} \Sigma_{2}, k\right) .
$$

Hence, $\mathscr{H}_{\mathrm{Sch}}$ is smooth and admissible. By the Stone-von Neumann theorem (Theorem 4.15), $\mathscr{H}_{\mathrm{Sch}}$ is isomorphic to a Heisenberg representation tensored with a $k$-vector space. But the fact that $\operatorname{dim}_{k} \mathscr{H}_{\mathrm{Sch}}^{p^{n} T_{p} \Sigma}=p^{2 n}$ shows that the latter vector space has dimension 1 . Hence, $\mathscr{H}_{\mathrm{Sch}}$ is itself a Heisenberg representation.

We introduce an ind $k$-group scheme

$$
P:=\left\{\left(\begin{array}{cc}
\left(B^{\vee}\right)^{-1} & C \\
0 & B
\end{array}\right) \mid B \in \underline{\operatorname{Aut}}\left(V_{p} \Sigma_{2}\right), C \in \underline{\operatorname{Hom}}\left(V_{p} \Sigma_{1}, V_{p} \Sigma_{2}\right), C^{\vee}=C\right\} .
$$

(The dual $\vee$ between $V_{p} \Sigma_{1}$ and $V_{p} \Sigma_{2}$ is taken with respect to $\hat{e}_{p}^{L}$.) Once a basis is chosen, we can identify $\underline{\operatorname{Aut}}\left(V_{p} \Sigma_{2}\right) \simeq G L_{g}\left(\mathbb{Q}_{p}\right)$ and $\underline{\operatorname{Hom}}\left(V_{p} \Sigma_{1}, V_{p} \Sigma_{2}\right) \simeq$ $M_{g}\left(V_{p} \mu_{p^{\infty}}\right)$ in view of (7-6) below. (We apologize for two different usages of $M_{g}$.)

Corollary 7.7. In the setting of Proposition 7.5, we have

(i) a canonical isomorphism $\underline{\mathrm{Sp}}\left(V_{p} A, \hat{e}_{p}^{L}\right) \simeq P$ as group functors and

(ii) $\mathbb{G}_{m} \times P \simeq \operatorname{Mp}\left(V_{p} A, \hat{e}_{p}^{L}\right)$ as group functors via $(\lambda, g) \mapsto \lambda M_{g}$, where $M_{g}$ is defined on $\overline{\mathcal{H}_{\mathrm{Sch}}}$ as

$$
\begin{aligned}
\text { - } g & =\left(\begin{array}{cc}
\left(B^{\vee}\right)^{-1} & 0 \\
0 & B
\end{array}\right),\left(M_{g} \phi\right)(x)=\phi\left(B^{-1} x\right) . \\
\text { - } g & =\left(\begin{array}{cc}
I & C \\
0 & I
\end{array}\right),\left(M_{g} \phi\right)(x)=\hat{e}_{p}^{L}(C x, x) \phi(x) .
\end{aligned}
$$


Remark 7.8. The action in (ii) above is the same as (i) and (ii) of Proposition 7.1. Since $\operatorname{Sp}\left(V_{p} A, \hat{e}_{p}^{L}\right)$ is smaller when $\operatorname{char}(k)=p$, the action (iii) simply does not show up here. Also note that the above $M_{g}$-action does not involve $|\operatorname{det} B|_{p}^{-1 / 2}$, which does not make sense in $k$.

Proof. Part (i) is derived from the canonical isomorphisms

$$
\begin{gathered}
\underline{\operatorname{Hom}}_{k}\left(\mathbb{Z} / p^{n} \mathbb{Z}, \mathbb{Z} / p^{n} \mathbb{Z}\right) \simeq \mathbb{Z} / p^{n} \mathbb{Z}, \quad \underline{\operatorname{Hom}}_{k}\left(\mathbb{Z} / p^{n} \mathbb{Z}, \mu_{p^{n}}\right) \simeq \mu_{p^{n}}, \\
\underline{\operatorname{Hom}}_{k}\left(\mu_{p^{n}}, \mathbb{Z} / p^{n} \mathbb{Z}\right)=0, \quad \underline{\operatorname{Hom}}_{k}\left(\mu_{p^{n}}, \mu_{p^{n}}\right) \simeq \mathbb{Z} / p^{n} \mathbb{Z} .
\end{gathered}
$$

For (ii), the given action of $(\lambda, g)$ obviously defines a splitting of (5-3). Since $\mathbb{G}_{m} \times \underline{\operatorname{Sp}}\left(V_{p} A, \hat{e}_{p}^{L}\right)$ is representable by a $k$-group scheme, the same is true for $\underline{\mathrm{Mp}}\left(V_{p} \bar{A}, \hat{e}_{p}^{L}\right)$.

Remark 7.9. If one naïvely attempts to find a mod $p$ Weil representation, then one could guess that $C_{c}^{\infty}\left(\mathbb{Q}_{p}^{g}, \overline{\mathbb{F}}_{p}\right)$ is the right model just by imitating the classical Schrödinger model without using the Heisenberg representation (which may be difficult to come up with unless the Heisenberg group is defined scheme-theoretically). But then one gets into trouble in defining a projective representation of $\operatorname{Sp}_{2 g}\left(\mathbb{Q}_{p}\right)$. Indeed, the group action in (iii) of Proposition 7.1, which amounts to the Fourier transform, does not make sense over $\overline{\mathbb{F}}_{p}$. (For instance, there is no $\overline{\mathbb{F}}_{p}$-valued Haar measure on $V_{2}$.) The virtue of our scheme-theoretic approach is that it renders a precise meaning to $C_{c}^{\infty}\left(\mathbb{Q}_{p}^{g}, \overline{\mathbb{F}}_{p}\right)$, which is but a special case of a mod $p$ Weil representation corresponding to the ordinary $p$-divisible group. In addition, our approach explains why the Fourier transform action should disappear from the picture.

Denote by $D^{\infty}\left(V_{p} \Sigma_{1}, k\right)$ the dual $k$-vector space of $C^{\infty}\left(V_{p} \Sigma_{1}, k\right)$. The following proposition allows us to transport the Heisenberg representation structure from $C^{\infty}\left(V_{p} \Sigma_{1}, k\right)$ to $D^{\infty}\left(V_{p} \Sigma_{1}, k\right)$ :

Proposition 7.10. There is a canonical isomorphism of $k$-vector spaces

$$
C_{c}^{\infty}\left(V_{p} \Sigma_{2}, k\right) \simeq D^{\infty}\left(V_{p} \Sigma_{1}, k\right) .
$$

Proof. For a finite group scheme $G$ and its dual $G^{\vee}$ over $k$, recall the standard fact that their rings of functions are canonically $k$-dual, namely $\mathscr{O}_{G} \simeq\left(\mathscr{O}_{G^{\vee}}\right)^{\vee}$. When applied to $G=\frac{1}{p^{n}} \mathbb{Z}_{p} / \mathbb{Z}_{p}$, this provides a canonical isomorphisms $C\left(\frac{1}{p^{n}} \mathbb{Z}_{p} / \mathbb{Z}_{p}, k\right) \simeq$ $D\left(\mu_{p^{n}}, k\right)$ for all $n \geq 1$, where $D$ denotes the distribution. By taking inverse limit, $C_{c}\left(\mathbb{Q}_{p} / \mathbb{Z}_{p}, k\right) \simeq D^{\infty}\left(T_{p} \mu_{p^{\infty}}, k\right)$. Now by taking the direct limit along the maps on $C_{c}$ and $D^{\infty}$ induced by

$$
\mathbb{Q}_{p} / \mathbb{Z}_{p} \stackrel{p}{\leftarrow} \mathbb{Q}_{p} / \mathbb{Z}_{p} \stackrel{p}{\leftarrow} \cdots, \quad T_{p} \mu_{p^{\infty}} \stackrel{p}{\rightarrow} T_{p} \mu_{p^{\infty}} \stackrel{p}{\rightarrow} \cdots,
$$

we obtain $C_{c}^{\infty}\left(\mathbb{Q}_{p}, k\right) \simeq D^{\infty}\left(V_{p} \mu_{p^{\infty}}, k\right)$. The same argument with multiple copies of $\mathbb{Q}_{p}$ and $V_{p} \mu_{p^{\infty}}$ proves the proposition. 
So far we have considered only ordinary $p$-divisible groups $\Sigma$. For a general $\Sigma$ with a complete polarization $\Sigma=\Sigma_{1} \times \Sigma_{2}$ with respect to $\hat{e}_{p}^{L}$ (where $C_{c}^{\infty}\left(V_{p} \Sigma_{2}, k\right.$ ) does not make sense), it remains to be answered whether $D^{\infty}\left(V_{p} \Sigma_{2}, k\right)$ is a Heisenberg representation.

Remark 7.11. When $p>2$, the material of this subsection can be rewritten in terms of only $\left(\Sigma,\langle\cdot, \cdot\rangle_{0}\right)$ by using Section $6 \mathrm{D}$, getting rid of $(A, L)$ from the picture. (See Remark 6.6 for $p=2$.) We retained $(A, L)$ to make the analogy with Section 7A more transparent and also not to make an exception $p \neq 2$.

7D. Over a ring of mixed characteristic $(0, p)$. In this final example, consider the case when:

- $K$ is a field extension of $\mathbb{Q}_{p}$ complete with respect to a $p$-adic valuation $v_{p}: K^{\times} \rightarrow \mathbb{R}$. Assume that $x^{p^{n}}-1$ splits completely in $K$ for all $n \geq 1$.

- $\mathscr{O}_{K}:=\left\{a \in K^{\times} \mid v_{p}(a) \geq 0\right\} \cup\{0\}$.

- $S=\operatorname{Spec}_{K}$.

- $\Sigma=\Sigma_{1} \times \Sigma_{2}$ with $\Sigma_{1}=\left(\mu_{p^{\infty}}\right)^{g}$ and $\Sigma_{2}=\left(\mathbb{Q}_{p} / \mathbb{Z}_{p}\right)^{g}$ over $S$.

$\cdot\langle\cdot, \cdot\rangle_{0}: \Sigma \times \Sigma \rightarrow \mu_{p}$ is a symplectic pairing sending

$$
\left.\left(\left(\left(x_{i}\right)_{i=1}^{g},\left(y_{i}\right)_{i=1}^{g}\right),\left(\left(x_{i}^{\prime}\right)_{i=1}^{g},\left(y_{i}^{\prime}\right)_{i=1}^{g}\right)\right)\right) \mapsto \prod_{i=1}^{g}\left(x_{i}, y_{i}^{\prime}\right) \prod_{i=1}^{g}\left(x_{i}^{\prime}, y_{i}\right)^{-1},
$$

where $(\cdot, \cdot): \mu_{p^{\infty}} \times \mathbb{Q}_{p} / \mathbb{Z}_{p} \rightarrow \mu_{p^{\infty}}$ is the canonical pairing and $\langle\cdot, \cdot\rangle$ is as in Section 6C.

- If $p=2$, assume that (7-3) holds with $T_{p} \Sigma_{1} \times T_{p} \Sigma_{2}$ in place of $\Lambda_{1} \oplus \Lambda_{2}$.

As in the previous subsection, define an ind-group scheme over $\mathbb{O}_{K}$ by

$$
P:=\left\{\left(\begin{array}{cc}
{ }^{t} B^{-1} & C \\
0 & B
\end{array}\right) \mid B \in G L_{g}\left(\mathbb{Q}_{p}\right), C \in \underline{\operatorname{Hom}}\left(\mathbb{Q}_{p}^{g},\left(V_{p} \mu_{p^{\infty}}\right)^{g}\right), C^{\vee}=C\right\} .
$$

Since (7-6) still holds with $\mathscr{O}_{K}$ in place of $k$, the exact analogue of Corollary 7.7 holds over $\mathscr{O}_{K}$. The $P$-representation on the free $\mathscr{O}_{K}$-module $\mathscr{H}_{\text {Sch, } \mathscr{O}_{K}}:=C_{c}^{\infty}\left(V_{p} \Sigma_{2}, \mathscr{O}_{K}\right)$ is the Weil representation. It is instructive to note how this specializes to Spec $K$ and $\operatorname{Spec} k$, where $k$ now denotes the residue field of $K$. By passing to $\operatorname{Spec} k$, we recover the Weil representation of Corollary 7.7, which is again a $P$-representation. Over the generic fiber, $\Sigma_{1}$ becomes isomorphic to $\left(\mathbb{Q}_{p} / \mathbb{Z}_{p}\right)^{g}$ noncanonically. Therefore, $\operatorname{Sp}\left(V_{p} \Sigma,\langle\cdot, \cdot\rangle_{0}\right)(K)$ is isomorphic to $\operatorname{Sp}_{2 g}\left(\mathbb{Q}_{p}\right)$. The Weil representation $C_{c}^{\infty}\left(\overline{V_{p}} \Sigma_{2}, K\right)$ over the generic fiber is the classical one described in Section 7A and contains $\mathscr{H}_{\mathrm{Sch}, \mathscr{C}_{K}}$ as an "integral model". This example illustrates that the integral model may admit a smaller action than the generic fiber. It would be worthwhile to 
describe a similar phenomenon for Weil representations in the case of nonordinary $p$-divisible groups over $\mathrm{O}_{K}$.

\section{Acknowledgments}

It is a great pleasure to acknowledge my huge debt to Mumford's papers and the Tata lectures [Mumford 2007]. I also learned a lot from reading Harris's manuscript [Harris 1987] and Moret-Bailly [Moret-Bailly 1985].

I appreciate Jeehoon Park for sparking my interest in the $\bmod p$ and $p$-adic theta correspondence. Wee Teck Gan and Atsushi Ichino educated me on the classical theory of theta, without which I could not initiate my project. I thank Benedict Gross and Guy Henniart for their interest and encouragement. I am grateful to Pierre Deligne for his generous advice on various points, which in particular led me to correct some errors. I thank Michael Harris, Kai-Wen Lan, Marc-Hubert Nicole, Junecue Suh and Martin Weissman for kindly answering my questions. I also benefited from conversations with Matthew Emerton, Minhyong Kim, Robert Kottwitz, Tetsushi Ito, Alberto Minguez and Teruyoshi Yoshida. Part of work was completed during my stay at the Institute for Advanced Study, where it is always wonderful to conduct research.

\section{References}

[Bosch et al. 1990] S. Bosch, W. Lütkebohmert, and M. Raynaud, Néron models, Ergebnisse der Math. (3) 21, Springer, Berlin, 1990. MR 91i:14034 Zbl 0705.14001

[Demazure 1972] M. Demazure, Lectures on p-divisible groups, Lecture Notes in Mathematics 302, Springer, Berlin, 1972. MR 49 \#9000 Zbl 0247.14010

[Faltings and Chai 1990] G. Faltings and C.-L. Chai, Degeneration of abelian varieties, Ergebnisse der Math. (3) 22, Springer, Berlin, 1990. MR 92d:14036 Zbl 0744.14031

[van der Geer and Moonen $\geq 2012$ ] G. van der Geer and B. Moonen, "Abelian varieties", preprint, available at http://staff.science.uva.nl/ bmoonen/boek/BookAV.html.

[Grothendieck 1963] A. Grothendieck, "Éléments de géométrie algébrique. III. Étude cohomologique des faisceaux cohérents. II”, Inst. Hautes Études Sci. Publ. Math. 17 (1963), 91. MR 29 \#1210 Zbl 0122.16102

[Grothendieck 1964] A. Grothendieck, "Éléments de géométrie algébrique, IV: Étude locale des schémas et des morphismes de schémas, I", Inst. Hautes Études Sci. Publ. Math. 20 (1964), 259. MR 30 \#3885 Zbl 0136.15901

[Gurevich and Hadani 2007] S. Gurevich and R. Hadani, "The geometric Weil representation", Selecta Math. (N.S.) 13:3 (2007), 465-481. MR 2009e:11078 Zbl 1163.22004

[Harris 1987] M. Harris, "Arithmetic of the oscillator representation", unpublished, 1987, available at http://people.math.jussieu.fr/ harris/Arithmetictheta.pdf.

[Harris and Taylor 2001] M. Harris and R. Taylor, The geometry and cohomology of some simple Shimura varieties, Annals of Mathematics Studies 151, Princeton University Press, 2001. MR 2002m:11050 Zbl 1036.11027 
[Hartshorne 1977] R. Hartshorne, Algebraic geometry, Graduate Texts in Mathematics 52, Springer, New York, 1977. MR 57 \#3116 Zbl 0367.14001

[Hida 2004] H. Hida, p-adic automorphic forms on Shimura varieties, Springer, New York, 2004. MR 2005e:11054 Zbl 1055.11032

[Katz and Mazur 1985] N. M. Katz and B. Mazur, Arithmetic moduli of elliptic curves, Annals of Mathematics Studies 108, Princeton University Press, 1985. MR 86i:11024 Zbl 0576.14026

[Kottwitz 1997] R. E. Kottwitz, "Isocrystals with additional structure. II”, Compositio Math. 109:3 (1997), 255-339. MR 99e:20061 Zbl 0966.20022

[Kudla 2002] S. S. Kudla, "Derivatives of Eisenstein series and arithmetic geometry", pp. 173-183 in Proceedings of the International Congress of Mathematicians, Vol. II (Beijing, 2002), edited by T. Li, Higher Ed. Press, Beijing, 2002. MR 2003k:11069 Zbl 1051.11029

[Lafforgue and Lysenko 2009] V. Lafforgue and S. Lysenko, "Geometric Weil representation: local field case”, Compos. Math. 145:1 (2009), 56-88. MR 2010c:22024 Zbl 1220.22015

[Lysenko 2006] S. Lysenko, "Moduli of metaplectic bundles on curves and theta-sheaves", Ann. Sci. École Norm. Sup. (4) 39:3 (2006), 415-466. MR 2008d:14019 Zbl 1111.14029

[Mœglin et al. 1987] C. Mœglin, M.-F. Vignéras, and J.-L. Waldspurger, Correspondances de Howe sur un corps p-adique, Lecture Notes in Mathematics 1291, Springer, Berlin, 1987. MR 91f:11040 Zbl 0642.22002

[Moret-Bailly 1985] L. Moret-Bailly, Pinceaux de variétés abéliennes, Astérisque 129, Société mathématique de France, Paris, 1985. MR 87j:14069 Zbl 0595.14032

[Mumford 1966] D. Mumford, "On the equations defining abelian varieties. I", Invent. Math. 1 (1966), 287-354. MR 34 \#4269 Zbl 0219.14024

[Mumford 1967a] D. Mumford, "On the equations defining abelian varieties. II", Invent. Math. 3 (1967), 75-135. MR 36 \#2621 Zbl 0219.14024

[Mumford 1967b] D. Mumford, “On the equations defining abelian varieties. III", Invent. Math. 3 (1967), 215-244. MR 36 \#2622 Zbl 0219.14024

[Mumford 1974] D. Mumford, Abelian varieties, 2nd ed., Oxford University Press, London, 1974. MR 2010e:14040 Zbl 0326.14012

[Mumford 2007] D. Mumford, Tata lectures on theta. III, Birkhäuser, Boston, MA, 2007. Reprint of the 1991 original. MR 2007k:14088 Zbl 1124.14043

[Mumford et al. 1994] D. Mumford, J. Fogarty, and F. Kirwan, Geometric invariant theory, 3rd ed., Ergebnisse der Math. (2) 34, Springer, Berlin, 1994. MR 95m:14012 Zbl 0797.14004

[Oort 2001] F. Oort, "Newton polygon strata in the moduli space of abelian varieties", pp. 417-440 in Moduli of abelian varieties (Texel Island, Netherlands, 1999), edited by C. Faber et al., Progr. Math. 195, Birkhäuser, Basel, 2001. MR 2002c:14069 Zbl 1086.14037

[Park 2010] J. Park, " $p$-adic family of half-integral weight modular forms via overconvergent Shintani lifting”, Manuscripta Math. 131:3-4 (2010), 355-384. MR 2011d:11104 Zbl 1221.11115

[Ramsey 2009] N. Ramsey, “The overconvergent Shimura lifting”, Int. Math. Res. Not. 2009:2 (2009), 193-220. MR 2010i:11060 Zbl 1165.11045

[Rapoport 2005] M. Rapoport, "A guide to the reduction modulo $p$ of Shimura varieties", pp. 271318 in Automorphic forms. I (Paris, 2000), edited by J. Tilouine et al., Astérisque 298, Société Mathématique de France, Paris, 2005. MR 2006c:11071 Zbl 1084.11029

[Sekiguchi 1977] T. Sekiguchi, "On projective normality of Abelian varieties. II", J. Math. Soc. Japan 29:4 (1977), 709-727. MR 56 \#15662 Zbl 0355.14017 
[Shin $\geq 2012]$ S. W. Shin, "Geometric reductive dual pairs and a mod $p$ theta correspondence", preprint, available at http://math.mit.edu/ swshin/modpTheta.pdf.

[Stevens 1994] G. Stevens, " $\Lambda$-adic modular forms of half-integral weight and a $\Lambda$-adic Shintani lifting”, pp. 129-151 in Arithmetic geometry (Tempe, ZA, 1993), edited by N. Childress and J. W. Jones, Contemp. Math. 174, Amer. Math. Soc., Providence, RI, 1994. MR 95h:11051 Zbl 0869.11042

[Tate 1997] J. Tate, "Finite flat group schemes", pp. 121-154 in Modular forms and Fermat's last theorem (Boston, 1995), edited by G. Cornell et al., Springer, New York, 1997. MR 1638478 Zbl 0924.14024

Communicated by Bjorn Poonen

Received 2011-05-31 Revised 2011-09-25 Accepted 2011-10-26

swshin@math.mit.edu

Department of Mathematics,

Massachusetts Institute of Technology, 77 Massachusetts Ave, Cambridge, MA 02139, United States 


\section{Algebra \& Number Theory}

msp.berkeley.edu/ant

\section{EDITORS}

MANAGING EDITOR

Bjorn Poonen

Massachusetts Institute of Technology

Cambridge, USA

\author{
EDITORIAL BOARD CHAIR \\ David Eisenbud \\ University of California \\ Berkeley, USA
}

\section{BOARD OF EDITORS}

Georgia Benkart

Dave Benson

Richard E. Borcherds

John H. Coates

J-L. Colliot-Thélène

Brian D. Conrad

Hélène Esnault

Hubert Flenner

Edward Frenkel

Andrew Granville

Joseph Gubeladze

Ehud Hrushovski

Craig Huneke

Mikhail Kapranov

Yujiro Kawamata

János Kollár

Yuri Manin

Barry Mazur

Philippe Michel
University of Wisconsin, Madison, USA

University of Aberdeen, Scotland

University of California, Berkeley, USA

University of Cambridge, UK

CNRS, Université Paris-Sud, France

University of Michigan, USA

Freie Universität Berlin, Germany

Ruhr-Universität, Germany

University of California, Berkeley, USA

Université de Montréal, Canada

San Francisco State University, USA

Hebrew University, Israel

University of Virginia, USA

Yale University, USA

University of Tokyo, Japan

Princeton University, USA

Northwestern University, USA

Harvard University, USA

École Polytechnique Fédérale de Lausanne
Susan Montgomery

Shigefumi Mori

Raman Parimala

Jonathan Pila

Victor Reiner

Karl Rubin

Peter Sarnak

Joseph H. Silverman

Michael Singer

Vasudevan Srinivas

J. Toby Stafford

Bernd Sturmfels

Richard Taylor

Ravi Vakil

Michel van den Bergh

Marie-France Vignéras

Kei-Ichi Watanabe

Andrei Zelevinsky

Efim Zelmanov
University of Southern California, USA

RIMS, Kyoto University, Japan

Emory University, USA

University of Oxford, UK

University of Minnesota, USA

University of California, Irvine, USA

Princeton University, USA

Brown University, USA

North Carolina State University, USA

Tata Inst. of Fund. Research, India

University of Michigan, USA

University of California, Berkeley, USA

Harvard University, USA

Stanford University, USA

Hasselt University, Belgium

Université Paris VII, France

Nihon University, Japan

Northeastern University, USA

University of California, San Diego, USA

\section{PRODUCTION}

production@msp.org

Silvio Levy, Scientific Editor

See inside back cover or www.jant.org for submission instructions.

The subscription price for 2012 is US \$175/year for the electronic version, and \$275/year ( $\$ 40$ shipping outside the US) for print and electronic. Subscriptions, requests for back issues from the last three years and changes of subscribers address should be sent to Mathematical Sciences Publishers, Department of Mathematics, University of California, Berkeley, CA 94720-3840, USA.

Algebra \& Number Theory (ISSN 1937-0652) at Mathematical Sciences Publishers, Department of Mathematics, University of California, Berkeley, CA 94720-3840 is published continuously online. Periodical rate postage paid at Berkeley, CA 94704, and additional mailing offices.

ANT peer review and production are managed by EditFLOW ${ }^{\circledR}$ from Mathematical Sciences Publishers.

PUBLISHED BY

mathematical sciences publishers

http://msp.org/

A NON-PROFIT CORPORATION

Typeset in IATEX

Copyright ( 2012 by Mathematical Sciences Publishers 


\section{Algebra \& Number Theory}

Volume $6 \quad$ No. $8 \quad 2012$

On the refined ramification filtrations in the equal characteristic case

LIANG XIAO

On common values of $\phi(n)$ and $\sigma(m)$, II

1669

KEVIN FORD and PAUL POLLACK

Galois representations associated with unitary groups over $\mathbb{Q}$

CHRISTOPHER SKINNER

Abelian varieties and Weil representations

SUG WOO SHIN

Small-dimensional projective representations of symmetric and alternating groups

Alexander S. KleshcheV and Pham HuU TieP

Secant varieties of Segre-Veronese varieties

1817

Claudiu Raicu 